\title{
SUB-OPTIMAL AND ROBUST DOWNLINK BEAMFORMING IN WIRELESS NETWORKS WITH IMPERFECT CHANNEL STATE INFORMATION
}

A Dissertation presented to

the Faculty of the Graduate School

at the University of Missouri

In Partial Fulfillment

of the Requirements for the Degree

Doctor of Philosophy

by

AHMED ABID-AWN AL-ASADI

Dr. Naz Islam, Dissertation Supervisor

MAY 2021 
The undersigned, appointed by the Dean of the Graduate School, have examined the dissertation entitled:

\section{SUB-OPTIMAL AND ROBUST DOWNLINK BEAMFORMIN IN WIRELESS NETWORKS WITH IMPERFECT CHANNEL STATE INFORMATION}

presented by Ahmed Abid-Awn Al-Asadi, a candidate for the degree of Doctor of Philosophy and hereby certify that, in their opinion, it is worthy of acceptance.

\begin{tabular}{c}
\hline Dr. Naz Islam \\
\hline Dr. Robert Oconell \\
\hline Dr. John Gahl \\
\hline
\end{tabular}

Dr. Ronald McGarvey 


\section{Dedicated to}

The awaited Saviour,

Mohammad Ibn Al-Hasan Al-Mahdi 


\section{ACKNOWLEDGMENTS}

First of all, I would like to thank my Lord, my creator, Almighty Allah for his benefactions, blessings and mercy. Without his everlasting support and custody, I would never complete my PhD degree successfully. O' my Lord, I cannot achieve thanksgiving as my thanking You lacks thanksgiving!

I would like to express my gratitude toward my sponsor, the ministry of higher education and scientific research in Iraq. MOHSER has granted me a six-years scholarship to conduct my $\mathrm{PhD}$ study in US, and provided me consistent fund despite economical, political and military crises there in Iraq. Thanks MOHSER for making my dream of studying abroad come true!

I would like to express my sincere gratitude and appreciation to my friend, research adviser and mentor Prof. Naz Islam. Working with Dr.Islam has proven to be one of the best things I have ever made. Dr.Islam has believed in me and considered my success (or fail) is his success (or fail). He has helped me to strive harder to develop better ideas and to become an outstanding researcher. He has always been available to sit with me and listen to my academic and personal issues. Being under his supervision will be one of the things that I shall always be proud of. Thanks Dr. Islam!

I would like to thank Dr. Robert O'conell, Dr. John Gahl, and Dr. Ronald McGarvey for serving in my PhD committee. Special thanks go to the Electrical and Computer Engineering department and the nice faculty and staff there who make my PhD studying at MU fruitful and delightful. Thanks Mizzou!

During my staying at MU, I have come across a lot of colleagues and met real 
friends who have provided me support and created a very warm and pleasant community around me. I am very indebted to my amazing friends in Kumal's, Imam Hasan's, and "Duhaa and Zeara" groups who have shared with me my happiness, sadness and frequent occasions. Participation in their weekly gathering has relieved my studying stress and indeed sharpened my personal traits. Big thank goes to them!

Finally, the words cannot ever express my feelings toward my big family: my wife and kids. Their love, care, understanding, patience, and unlimited support have made my doctoral course of study much easier and successful. Special thank goes to my life partner, Zainab, who walks down beside me in my tough road! 
TABLE OF CONTENTS

ACKNOWLEDGMENTS ................ ii

LIST OF TABLES $\ldots \ldots \ldots \ldots \ldots \ldots$ viii

LIST OF FIGURES $\ldots \ldots \ldots \ldots \ldots \ldots \ldots \ldots$ ix

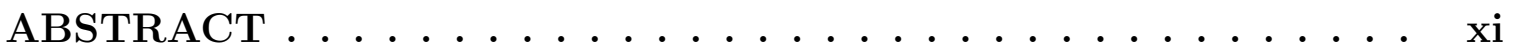

\section{CHAPTER}

1 Introduction ...................... 1

1.1 Notation and Acronyms . . . . . . . . . . . . . . . . 1

1.2 Introduction . . . . . . . . . . . . . . . . . 4

1.3 Wireless Sensor Network $(\mathrm{WSN}) \ldots \ldots \ldots \ldots \ldots$

1.4 WSN Lifespan . . . . . . . . . . . . . . . . . . . . 6

1.5 Energy Harvester . . . . . . . . . . . . . . . . . . . . 8

1.6 Wireless Power Transfer(WPT) . . . . . . . . . . . . . . . 10

1.7 Research Contribution . . . . . . . . . . . . . . . . . . 11

1.8 Dissertation Organization $\ldots \ldots \ldots \ldots \ldots$

2 Theoretical Background $\ldots \ldots \ldots \ldots \ldots \ldots$

2.1 Wireless Propagation . . . . . . . . . . . . . . . . 14

2.2 MIMO Channel State Information(CSI) $\ldots \ldots \ldots \ldots \ldots$

$2.2 .1 \quad$ Spatial Multiplexing $\ldots \ldots \ldots \ldots \ldots \ldots$

$2.2 .2 \quad$ Spatial Diversity $\ldots \ldots \ldots \ldots \ldots$ 
2.3 CSI Uncertainty $\ldots \ldots \ldots \ldots$

2.4 Cognitive Radio Network . . . . . . . . . . . . . . . . . . . . 21

2.4.1 Interweave Cognitive Network . . . . . . . . . . . . . 24

2.4 .2 Underlay Cognitive Network . . . . . . . . . . . . . . . . 24

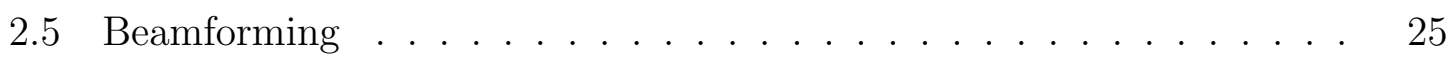

2.5 .1 Number of user . . . . . . . . . . . . . . . . 27

2.5 .2 direction . . . . . . . . . . . . . . . . . . . . 29

2.5.3 Type Of Communication Network . . . . . . . . . . . . . 29

2.5 .4 CSI Accuracy . . . . . . . . . . . . . . . 30

2.6 Convex Optimization . . . . . . . . . . . . . . . . . . . . . . . 31

2.7 Successive Convex Approximation . . . . . . . . . . . . . . . . 32

2.8 Lagrange duality $\ldots \ldots \ldots \ldots \ldots \ldots$

2.9 Successive Convex Approximation . . . . . . . . . . . . . . 34

2.10 bisection search method $\ldots \ldots \ldots \ldots \ldots$

3 Worst Case Fair Beamforming for Multiple Multicast Groups in Multicell Networks . . . . . . . . . . . . . 40

3.1 Introduction . . . . . . . . . . . . . . . . 40

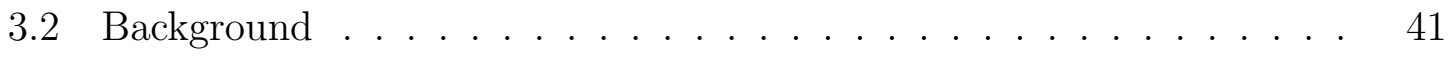

3.3 System model and Problem Formulation $\ldots \ldots \ldots \ldots$

3.3 .1 System Model . . . . . . . . . . . . . . . . . . . . 46

3.3 .2 Problem formulation . . . . . . . . . . . . . . . 48

3.4 Suboptimal solution . . . . . . . . . . . . . . . . . . . . . 51

3.5 Proposed(Robust) solution . . . . . . . . . . . . . . . . . 53 
3.5.1 Solution

3.5.2 Solution for

for
$\{\boldsymbol{\Delta} \mathbf{R}\}_{m=1}^{M} 54$

$$
\{\mathbf{w}\}_{k=1}^{K} 56
$$

3.6 Numerical Results . . . . . . . . . . . . . . . . . . . . . . . . . . . 58

3.7 Conclusion . . . . . . . . . . . . . . . . . . . . . . 63

3.8 Appendices . . . . . . . . . . . . . . . . . . . . . . . . . . . . 64

3.8.1 Appendix 1: proof for(3.17),(3.18) and (3.23) . . . . . 64

3.8.2 Appendix 2: proof of feasibility and convergence of P8 . . . 66

4 Robust Underlay Cognitive Network Download Beamforming In Multiple Users,Multiple Groups Multicell Scenario . . . . . . . 69

4.1 Introduction . . . . . . . . . . . . . . . . . . . . . . . 69

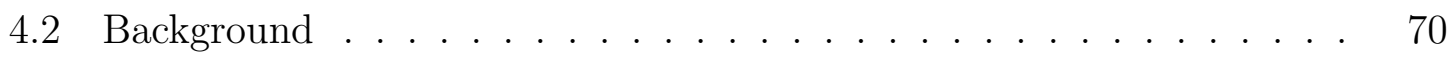

4.3 Chapter orgnization And Notation $\ldots \ldots \ldots \ldots . \ldots . \ldots 74$

4.4 System model and problem statement . . . . . . . . . . . 75

4.5 Worst case approach $\ldots \ldots \ldots \ldots \ldots \ldots$. . . . . . . . . . 82

4.6 Robust solution . . . . . . . . . . . . . . . . . . . . . . 86

4.7 Simulation results . . . . . . . . . . . . . . . . . . . 93

4.8 Conclusion . . . . . . . . . . . . . . . . . . . . . 100

4.9 Appendices . . . . . . . . . . . . . . . . . . . . . . . . . 101

4.9.1 Appendix 1: proof of feasibility and convergence of P4 . . . 101

4.9.2 APPENDIX 2: (Solution for P5 in section 4 to find $\{\boldsymbol{\Delta} \mathbf{R}\}_{m=1}^{M} 104$

5 Summary and Future Work . . . . . . . . . . . . 106

5.1 Research Summary . . . . . . . . . . . . . . . . . . 106 
5.2 Future Work . . . . . . . . . . . . . . . . . 107

5.2.1 Channel Estimation and Channel Uncertainty . . . . . . . . 107

5.2.2 Non Orthogonal Multiple Access(NOMA) . . . . . . . . . . . 108

5.2.3 Cognitive Network and Model Order Selection . . . . . . . . . 108

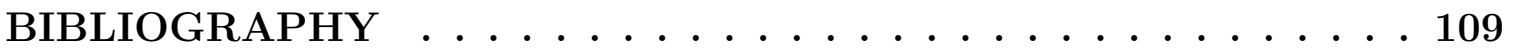

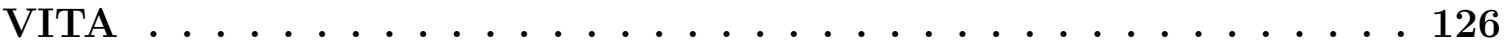




\section{LIST OF TABLES}

Table $\quad$ Page

3.1 Minimum SINR versus number of base station for three cases . . . 63 


\section{LIST OF FIGURES}

Figure

Page

1.1 Wireless Sensor Network block diagram $[4] \ldots \ldots \ldots \ldots$

1.2 Percentage of power consumption in each prat of wireless sensor node [10]. 8

1.3 Block diagram of wireless sensor node with energy harvester [16]. . . 9

1.4 Power transmission by beamforming technique [21] . . . . . . . . 11

2.1 Different propagation phenomenons in wireless communication system. 16

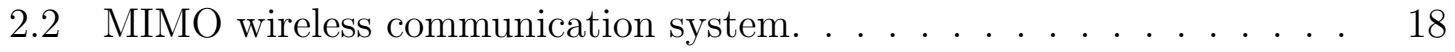

2.3 Utilization of frequency resource in wireless communication. . . . . 22

2.4 Type of sharing technique. . . . . . . . . . . . . . . . 23

2.5 Category of different beamforming in wireless communication system. 26

2.6 Single user baemforming IN MIMO system. . . . . . . . . . 27

2.7 multiuser baemforming IN MIMO system. $\ldots \ldots \ldots \ldots \ldots$

2.8 Convex and Non-convex set. . . . . . . . . . . . . . . . . . 32

2.9 strong and weak duality explanation. . . . . . . . . . 35

2.10 convergence of successive convex approximation method. . . . . . 37

2.11 Bisection search method starting interval. . . . . . . . . . 38

2.12 Bisection search end interval. . . . . . . . . . . . . . 39 
3.1 Multicast, mulicell, multigroup System model . . . . . . . . . . 47

3.2 Minimum SINR versus the number of iterations for $\mathrm{k}=2$ and 3 group 60

3.3 Minimum transmitted power versus minimum received SINR for different numbers of transmit antenna and different robust solutions . . 61

3.4 Minimum SINR versus normalized uncertainty . . . . . . . . . . . 61

3.5 Minimum SINR versus the number of users per group for three solutions 62

4.1 Cognitive Radio Multicast, Mulicell, Multigroup Wireless System Model 76

4.2 Minimum SINR for different solutions versus number of iteration $\mathbf{N}=$ $\mathbf{N}_{p u}=5, \mathbf{M}=12, \mathbf{k}=2, \sigma_{m}=1, \epsilon_{m}=\epsilon_{I b}=.01, P_{I P}$ and $P_{b}=20 d B .95$

4.3 Minimum SINR for different solutions versus feasibility . . . . . 96

4.4 Minimum SINR for different uncertainty ratio $\epsilon_{m}$ solutions versus num-

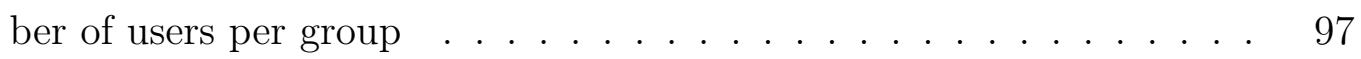

4.5 Minimum SINR for different solutions and different uncertainty radios $\epsilon_{m}$ versus minimum transmitted power $\ldots \ldots \ldots \ldots 9$

4.6 Minimum SINR for different solutions versus cumulative distribution function $(\mathrm{CDF})$ at SU-R . . . . . . . . . . . . . . 99

4.7 Interfernce power for different solutions versus cumulative distribution function $(\mathrm{CDF})$ at PU-R. . . . . . . . . . . . . 100 


\begin{abstract}
The rapid growth in internet applications such as video streaming enforce the researcher to explore a new wireless technique to ensure high signal to interference noise ratio (SINR) at the end users, leading to high quality of service (QOS). The fourth generation $(4 \mathrm{G})$ wireless technologies introduced a with promising technique known as multiple-input-multiple-output (MIMO) paradigm. The MIMO offers spatial diversity of multiple signals between the source and the destination which can ensure high concentration of the desired power at the destination as well as combat the unwanted interference which can be done by the beamforming technique, implemented in two ways the up-link and the down-link.

Two methods of beamforming have been addressed in MIMO wireless communications, the first consider the minimization of transmitted power for predefined SINR at the receiver and the second approach consider maximization of SINR at the recipient while maintaining the power at the sender to a small fixed value. Rigid beamforming is assured when the accurate channel state information (CSI) of the wireless system are acquired at the beamforming side. Because of some practical limitations in wireless systems such as feedback error, dynamic characteristics of wireless channel, etc., the ideal CSI cannot be obtained and thus the beamforming must consider the error in CSI. Three type of solutions have been developed to combat the effect of uncertain CSI these solutions are the non-robust, the sub-optimal and the robust solution.

In this work the sub-optimal and the robust downlink beamforming in conventional wireless network are addressed. The solution considers a multicast, multi-group, multicell scenario. The uncertainty in CSI is modeled mathematically using Frobinius
\end{abstract}


norm and the beamforming method used is the QOS method where the minimum SINR over all groups is maximized for small predefined transmitted power. Because the problem is difficult to be solved as a single optimization problem, it is divided into two problems. The first problem eliminates the effect of CSI uncertainty using the non-monotone spectral projected gradient (NMSPG) method, and the second problems use the successive convex approximation (SCA) method to extract the beamforming vectors for each group. The procedure goes through an iterate-alternative convex technique between the two methods until stopped by some predefined criteria.

Wireless communication researchers have also achieved significant development in the area of spectrum scarcity by introducing the cognitive radio (CR) network. In a CR network the secondary users (SUs) can utilize the licensed frequency that is underutilized by the primary users (PUs). Two type of CR network were developed, the overlay and the underlay CR network. The beamforming in an overlay CR network follows the same procedure as in a conventional network while in an underlay CR network an extra constraint must be added to the beamforming problem which makes the problem more difficult to solved.

In this thesis the beamforming problem in a CR network with multiple secondary transmitters that generate multiple beamforming vectors to multiple groups of secondary receivers under uncertain CSI are analyzed and solved. Two solutions were developed: the sub-optimal and the robust solutions. For the sub-optimal solution, the problem is split into two problems, the QOS and the interference power problem to combat the effect of CSI uncertainty then the two problems are combined to find the beamforming vectors using the SCA method. For the robust solution, the problem is also divided into two problems, the QOS and the interference power problem 
to eliminate the CSI uncertainty. The interference power problem is solved using the Lagrangian duality. The QOS problem is solved using the Lagrangian duality and the NMSPG method. after addressing CSI uncertainty, the beamforming vectors are extracted using the SCA method and, solved using the bisection search method. 


\section{Chapter 1}

\section{Introduction}

\subsection{Notation and Acronyms}

Throughout the dissertation, bold upper-case letters denote matrices and bold lowercase letters represent column vectors. Further notational symbols are

$\mathbb{C}^{m \times n}, \mathbb{H}^{n} \quad$ The complex space of $m \times n$ matrices and the space of Hermitian $n \times n$ matrices

$\mathbb{R}^{m \times n}, \mathbb{R}_{+}^{m \times n} \quad$ The real space of $m \times n$ matrices and of $m \times n$ matrices with non-negative entries

$(\cdot)^{\dagger}$

$(\cdot)^{t}$

$|\mathbf{A}|$

$\operatorname{Tr}(\mathbf{A})$ $[\mathbf{A}]_{i j}$
The Hermitian transpose

The regular transpose

Determinant of the square matrix $\mathbf{A}$

Trace of the matrix $\mathbf{A}$

The $i j$-th element of $\mathbf{A}$ 
$\lambda_{i}(\mathbf{A})$

$\mathbf{A} \succeq \mathbf{B}, \mathbf{A} \succ \mathbf{B}$

$\mathbf{A} \preceq \mathbf{B}$

$\operatorname{diag}(\mathbf{a})$

$\operatorname{Diag}(\mathbf{A})$

$\mathrm{U}_{\mathrm{A}} \Lambda_{\mathrm{A}} \mathrm{U}_{\mathrm{A}}^{\dagger}$

$\operatorname{vec}(\mathbf{A})$

$\|\mathbf{a}\|,\|\mathbf{a}\|_{\mathbf{X}}$

$\mathbb{E}[\cdot]$

$\|A\|_{p}$

$|a|$

$\log (\cdot)$

$\mathcal{C N}(\mathbf{m}, \mathbf{C})$

I

$a^{+}$

$\lceil a\rceil$
The $i$ th eignvalue of $\mathbf{A}$ such that $\lambda_{1}(\mathbf{A})$ denotes the maximum eigenvalue

The matrix $\mathbf{A}-\mathbf{B}$ is positive semi-definite or positive-definite

The matrix $\mathbf{A}-\mathbf{B}$ is negative semi-definite

Diagonal matrix formed by the elements of the vector a

Forms a diagonal matrix from the diagonal elements of $\mathbf{A}$

Eigenvalue decomposition, $\mathbf{U}_{\mathbf{A}} \in \mathbb{C}^{n \times n}$ is a unitary matrix and $\boldsymbol{\Lambda}_{\mathbf{A}}=\operatorname{diag}\left(\left[\lambda_{1}(\mathbf{A}) \cdots \lambda_{n}(\mathbf{A})\right]^{t}\right)$ with the eigenvalues arranged in non-increasing order

Stacks the columns of $\mathbf{A}$ into a single column

The Euclidean norm $\sqrt{\mathbf{a}^{\dagger} \mathbf{a}}$ and the weighted version $\sqrt{\mathbf{a}^{\dagger} \mathbf{X a}}$ for a Hermitian and positive semi-definite matrix $\mathbf{X}$ of an appropriate size

The statistical expectation

Schatten norm with order $p$

The absolute value of the scalar $a$

The natural logarithm

The distribution of a circularly symmetric complex Gaussian random vector with mean $\mathbf{m}$ and covariance $\mathbf{C}$

The identity matrix of an appropriate size

The maximum between $a$ and 0

denotes the least integer $\geq a$ 


\section{Acronyms}

Some of the abbreviations and acronyms we used in the dissertation are summarized as follows:

ACK/NAK ACKnowledgment and Negative AcKnowledgment signaling protocol

AI

AI-FDPS

$\mathrm{CC}$

CDMA

CR

CSCG

CSI

DSA

EVD

FBF

FDD

GLRT

HSDPA

IT

INR

LMI

LR

LTE-A

MI
Alternate-Iterate optimization

Alternate-Iterate Feasible Direction Projected Subgradient

Compound Capacity

Code-Division Multiple Access

Cognitive Radio

Circularly Symmetric Complex Gaussian distribution

Channel State Information

Dynamic Spectrum Access

Eigenvalue Decomposition

Fractional Bayes Factor

Frequency-Division Duplexing

Generalized Likelihood Ratio Test

High Speed Downlink Packet Access communication system

Interference Temperature

Interference-to-Noise Ratio

Linear Matrix Inequality

Leakage Rate

Long Term Evolution-Advanced communication system

Mutual Information 


$\begin{array}{ll}\text { MIMO } & \text { Multiple-Input Multiple-Output } \\ \text { NMSPG } & \text { Non-Monotone Spectral Projected Gradient } \\ \text { OFDM } & \text { Orthogonal Frequency-Division Multiplexing } \\ \text { PU } & \text { Primary User } \\ \text { QoS } & \text { Quality of Service } \\ \text { ROC } & \text { Receiver Operating Characteristic } \\ \text { SDP } & \text { Semi-Definite Programming } \\ \text { SINR } & \text { Signal-to-Interference-plus-Noise Ratio } \\ \text { SNR } & \text { Signal-to-Noise Ratio } \\ \text { SU } & \text { Secondary User } \\ \text { TDD } & \text { Time-Division Duplexing }\end{array}$

\subsection{Introduction}

The most important type of communication is the wireless communication which is begin with the invitation of electromagnetic waves (EM) by James Clerck Maxwell the Scottish Physics [1]. The EM is generated practically in Heinrich Hertiz Lab in 1885, then Alexander Stepanovich built the first radio receiver in 1894, after that in 1896 Guglielmo Marconi invented the first telegraph system [2]. Since that the people around the globe start to own wireless communication systems. In 1980 the most precious achievement in wireless communication is the wireless cellular communication which start to use massively around the world. The cellular wireless communication started as analogue system in $1 \mathrm{G}$ then developed to $2 \mathrm{G}$ through $3 \mathrm{G}$ and $4 \mathrm{G}$ and now 
reach to $5 \mathrm{G}$.

The advancement in cellular communication with new and wide users applications pushes a parallel development in digital electronic that made the hardware systems in a low price with higher efficiency. This make the wireless sensors and smart systems an important part of day uses. Furthermore, IEEE standards and embodiment of new technologies such as wireless sensor network (WSN), machine-to-machine(M2M), internet of thing (IOT) with cloud computing and big data have offered a new model that can authorize future communication network. Many communication technologies are more developed every day that change the working aspects and make the life more ease. These technologies propagate in different area such as environmental protection and monitoring, disaster expectation and prevention, social networking,etc.

\subsection{Wireless Sensor Network (WSN)}

The recent advancement in Miro-electromechanical systems (MEMS) technology and digital electronic leads to the development of high-efficiency, low-cost, and low-power integrated modules capable of sensing environmental and industrial phenomena, processing and transmission of data. This module is known as a wireless sensor node [3].

Using a huge amount sensor nodes to monitor single or multiple ambient situations depending on application leads to the design of a wireless sensor network (WSN) [4]. The network can in conventional or cognitive wireless scenarios $[5,6]$ depending on the method of utilization of spectrum used in the network.

The WSN has a wide range of application, for example, in military (such as targeting friendly forces and monitoring the enemy), in environmental (such as detecting Wildfires and monitoring wile animal), in healthcare (such as administration of a 
drug, and interfacing for disabled helping), etc. Because of the wide range of WSN applications, make it a part of day to make it a part of day to make it a part of day to make it a part of day-to-day smart monitoring is important part of day-to-day life.

Any wireless sensor node is comprising of the following units $[5,7,8]$ :

1) Data acquisition unit which comprises of the following parts:

a) Transducer.

b) Analog signal processing unit.

c) Analog to digital converter.

2) Memory and digital processing unit.

3) Transceiver unit.

4) Power generation and management unit.

The transducer converts the environmental phenomena to electrical signal processed by the analog signal processing unit to convert to a form acceptable by the analog to digital converter (ADC) to be converted to a digital signal. The processing unit is the heart part of the wireless sensor node that performs some digital processing operations and directs the storage and transfer unit data. The transceiver unit enable the node to communicate with other nodes in the network. As shown in figure $1.1[4]$ the power unit is connected to power each unit in the node, and any failure of the power unit leads to the node's discarding from the network.

\subsection{WSN Lifespan}

The most important factor that affects the lifetime of wireless sensor nodes and WSN is the energy supply. The energy supply should consider that the node(s) 


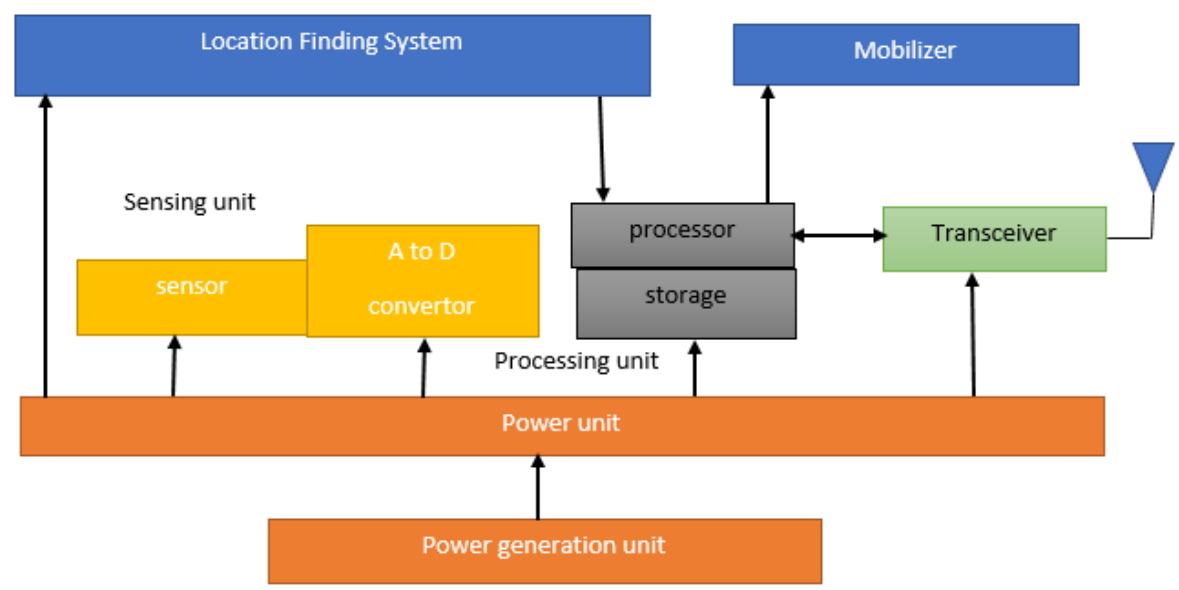

Figure 1.1: Wireless Sensor Network block diagram [4].

is(are) placed the and not attended for years, especially in security and environmental monitoring applications [9]. The ability of the node(s) to manage the energy supply is an important issue that must be considered in the design of the node(s).

A failure of the node(s) may cause the node(s) or some time the WSN to be discarded from the work, and in some applications, this is a Fatal issue, especially in the security system, leading to vulnerability.

To prolong the lifetime of the node or WSN, To prolong the lifetime of the node or WSN, understanding the power con- sumption in each part can lead to the node's optimal design. Figure 1.2 [10] shows the percentage of the power consumption in each part of the node. The researcher found that most of the power is utilized by the transceiver unit, making the node salient during the sleeping mode can extremely improve the node's lifespan.

Also, the researcher working hard to design algorithms, protocols [11,12] and improved hardware [13] to reduce the power consumption by the node. In the other 


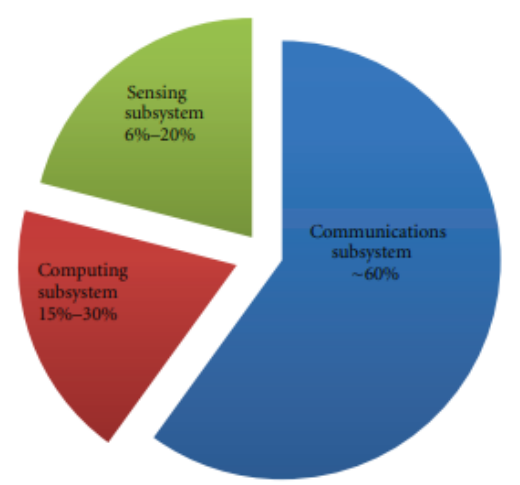

Figure 1.2: Percentage of power consumption in each prat of wireless sensor node [10].

side the network clustering and topology, which is depend on the application, is strongly affect the power consumption in the node. The researcher found the star topology network has more lifespan as compared with the mesh topology network [14]. Also, the network protocol has an effect, and the improvement of network protocol can enhance the power consumption in the WSN.

The method of powering the node can be classified into two types, the externally powered node and the self-powered node, and the use of each type depends on the application. The self-power node is used in remotely distributed nodes, and in this type of node, the use of energy harvesting to expand the lifetime of the node is applicable.

\subsection{Energy Harvester}

Energy scavenger or energy harvester is a system that can collect non-electrical energy and convert it to electrical energy. The energy harvester is used in self-powered node to prolong the lifespan of the node. The lifetime of the node can be extended to infinite as long as the non-electrical energy source is persisting. 


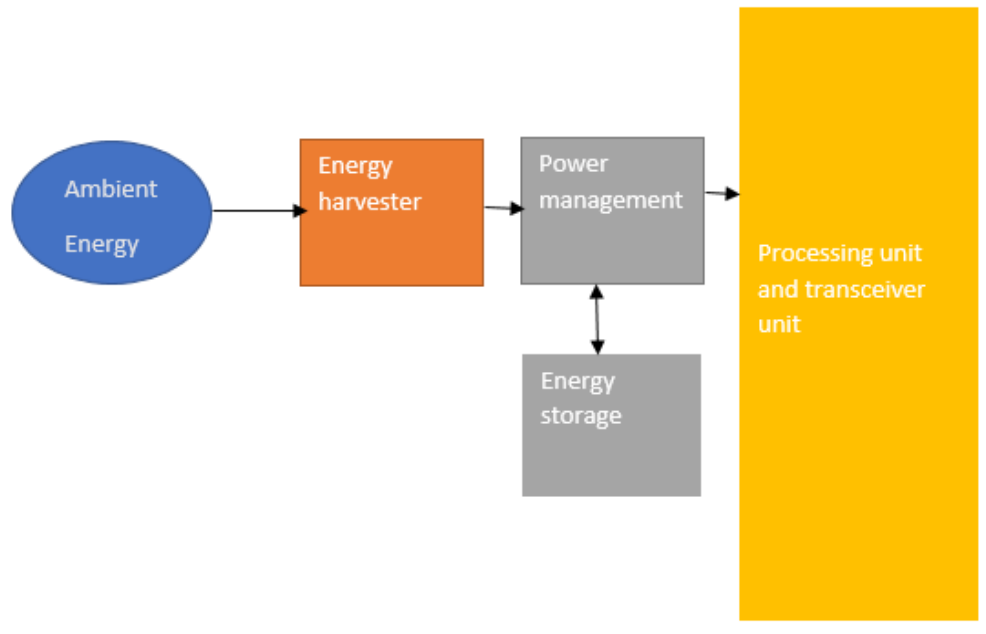

Figure 1.3: Block diagram of wireless sensor node with energy harvester [16].

Much researches have been done to locate the suitable energy harvester that can improve the node battery performance. the usual scavenge energy used in wireless sensor node include:

1) Solar energy (photovoltaic).

2) Mechanical energy(vibration).

3) electromagnetic radiation (wireless power transfer (WPT)).

The researchers advised the above three sources to be used in wireless sensor node since it is available everywhere and cannot be run out. The type of energy harvester used in the node depends on the application of WSN [15] and the specific part of the energy harvester in the wireless sensor node is shown in figure 1.3 [16]. The energy harvester is a part of a self-powered node used to support the energy storage device which is in the form of battery or storage capacitors. The energy harvesting devices are controlled by the power management unit, and the processing unit in node is responsiblefor the voltage stability in the node. 
The researchers found that the WPT energy harvesting device that employs magnetic field is the best harvesting method used to extend the lifetime in wireless sensor [17]. WPT energy harvester system is independent on the place of spreading the nodes also, its scale is small as compared with another energy harvester [18].

\subsection{Wireless Power Transfer(WPT)}

WPT is the promising technology that can be used to extend the battery life in wireless devices [19]. For wireless sensor node that placed remotely it can prolong the node's if the node consists of electromagnetic energy harvester. The first experiment to transfer power through the air by wireless method was by Nikole Tesla in 1914 when he transmitted signal in long island across Atlantic.

The WPT can be classified into three types [20]

1) Inductive coupling.

a-Magnetic resonance coupling.

b-Magmatic induction.

2) Electric induction. 3) Electromagnetic radiation.

a-Single-antenna source.

b-Multiple-antenna source.

Because of the limited distance to a maximum of few centimeters is not suitable for the application in WSN. Also, in the electric induction,, many things must be considered, such as the weather condition, which makes it unsuitable for the application in WSN that used in outdoor applications such as environmental applica- tions sand intrusion detection. Thus, only the electric induction and electromagnetic emission 

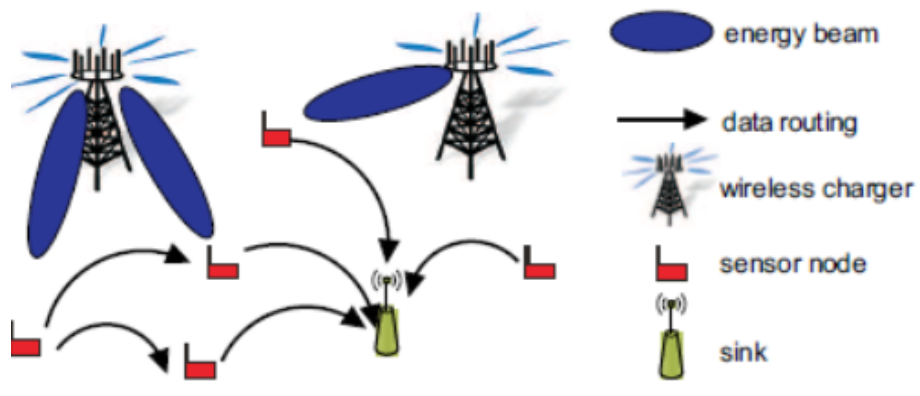

Figure 1.4: Power transmission by beamforming technique [21].

WPT are applicable for the application in WSN.

In this work we consider the electromagnetic emission and especially the multiple antenna scenario which is known as wireless energy transfer (WET). WET enable the transmitting of energy by the wireless electromagnetic beam to charge remote device [21]. The method invests the beamforming technique in multiple antenna system to supply power for long distant nodes as shown in figure 1.4 [21].

\subsection{Research Contribution}

The materials of this dissertation are based on the following papers:

[1] A. Al-Asadi, M. Al-Amidie, A. C. Micheas, R. G. McGarvey, N. E. Islam, Worst case fair beamforming for multiple multicast groups in multicell net- works, IET Communications 13 (6) (2018) 664-671.

[2] M. Al-Amidie, A. Al-Asadi, A. C. Micheas, N. E. Islam, Spectrum sensing based on Bayesian generalized likelihood ratio for cognitive radio systems with multiple 
antennas, IET Communications 13 (3) (2018) 305-311

[3] Ahmed Al-Asadi, Muthana Al-Amidie,Saddam K. Alwane Hayder M.Albehadili R. G. McGarvey, and N.E. Islam, "Robust Underlay Cognitive Network Download Beamforming In Multiple Users,Multiple Groups Multicell Scenario," IET Communications 14 (21) (2020) 3934-3943

[4] M. Al-Amidie, A. Al-Asadi,N. E. Islam, "Robust B-GLRT for Spectrum Sensing in MIMO Cognitive Radio Networks Under CSI Uncertainty," under review, MDPI electronic.

\subsection{Dissertation Organization}

The reminder chapters of the thesis are ordered as follows:

1) Chapter 2 describes the theoretical background related to the sub-optimal and robust downlink beamforming in wireless networks. Introducing the concept of MIMO in wireless communication and the role of CSI in the beamforming design also describe the effect of CSI uncertainty in the design. The chapter also, gives a brief introduction about convex optimization and optimization methods used in the dissertation.

2) 2) Chapter 3 describes the analysis and design of downlink beamforming vectors in the conventional wireless network in which the CSIs of all channel are imperfect by maximizing the SINR for limited transmitted power in the multiple-cell network SCA optimization method.

3) 3) Chapter 4 addresses the design of downlink beamforming vectors in cognitive wireless network using the same beamforming technique used in chapter 4 also, the CSIs are assumed imperfect. Two optimization methods are used to resolve the problem the SCA method and the binary search method. 
5) Finally, in chapter 5, the summarizes of the thesis are outlined, and some possible future research related to the thesis topic is discussed. 


\section{Chapter 2}

\section{Theoretical Background}

\subsection{Wireless Propagation}

The wireless signals transmitted from transmitter(s) to the receiver(s) and suffer

from several different phenomena [22-24] due to the nature of wireless signals and the propagation environments. These phenomena affect the communication and play an important role on the reliability and speed of the communication in any wireless communication systems. the phenomena are classified into:

1) Path losses $[25,26]$.

2) Fading and/or shadowing [27,28].

The path loss can describe the received power at the receiver in accordance to the transmitted power by the transmitter and its severely affected by the system parameter.

$$
\mathbf{P}_{r}=\mathbf{P}_{t} \mathbf{G}_{t} \mathbf{G}_{r}\left(\frac{\lambda}{2 \pi \mathbf{d}}\right)^{2}
$$


where $\mathbf{P}_{r}$ is the received power at the receiver, $\mathbf{P}_{t}$ is the transmitted power by the transmitter, $\mathbf{G}_{r}$ is the receiver antenna gain, $\mathbf{G}_{t}$ is the transmitter antenna gain, $d$ is the distance between the transmitter and the receiver, and $\lambda$ is the signal wave length which is proportional to the signal frequency. The simplest form of the signal path loss is the line of sight.

The fading can describe the fluctuation in the received signal which is divided into two type.

1)Fast fading.

2) Slow fading.

The fast fading as shown in figure 2.1 is accrue due to three different propagation affects :

1) Reflection.

2) Diffraction.

3) Scattering.

The fast fading take place when there is an obstacle between the transmitter and the receiver, and the length of this obstacle is small as compared with the signal wavelength. At the receiver there are multiple copies of the transmitted signal that have different time delay, different amplitude and/or different phase. These multiple copies can cause destructive or constructive of the final received signal by the receiver. Also, these signals can cause very high fluctuation over a short time in the received signal.

Slow fading that is also called large scale fading take place when there is an object between the transmitter and the receiver that cause one or both of the following affects. 


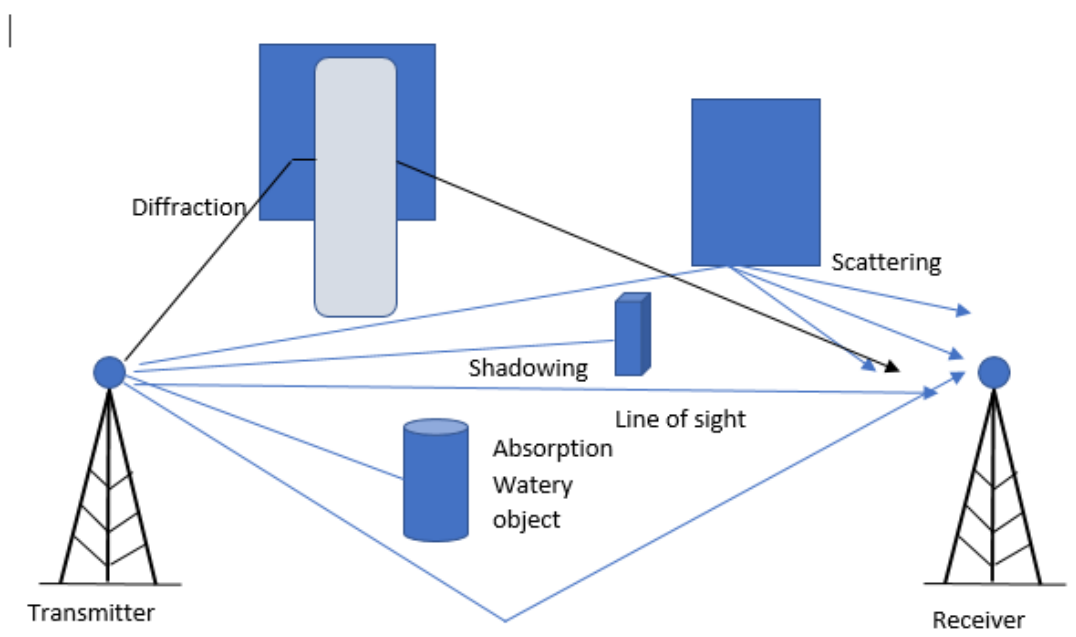

Figure 2.1: Different propagation phenomenons in wireless communication system.

1) Absorption.

2) Shadowing.

It happens when the object size is large as compared with the signal wavelength. Also, it is last for a long time such as several minutes or seconds.

\subsection{MIMO Channel State Information(CSI)}

MIMO open new era in wireless communication systems by improving the quality of service and the data rate at the end users [29] and this can support the high-quality multimedia transmission services for video and audio as well as support internet services. The MIMO enhances these new applications due to the main two important features offered by MIMO technology which are: 


\subsubsection{Spatial Multiplexing}

With this feature MIMO can transmit multiple streams of data at the same time instant by same frequency within same phase through several transmit antenna and can be received by several received antenna. this feature improves the capacity of the wireless communication system and leads to significant improvement in data rate because the data rate is directly proportional to the system capacity:

$$
C=B \log _{2}(1+S I N R)
$$

Where $C$ is the system capacity, $B$ is the system bandwidth that is related to the system rate, and SINR is the signal to inference noise ratio at the receiver.

\subsubsection{Spatial Diversity}

Choosing a correct antenna geometry at the transmitter and the receiver sides can significantly reduce the effect of correlation between the instantaneous channel coefficient seen by the transmitters and this can improve the signals quality at the receiver. With the reduction in correlation of channel and applies some advance techniques the signals at the receiver sides can be easily separated and this is one of the most important application of MIMO technology. The effect of correlation between channel coefficient can be reduced through orthogonally polarizing the antenna at both the receiver and transmitter.

The channel state information (CSI) severely effect the performance of the MIMO 


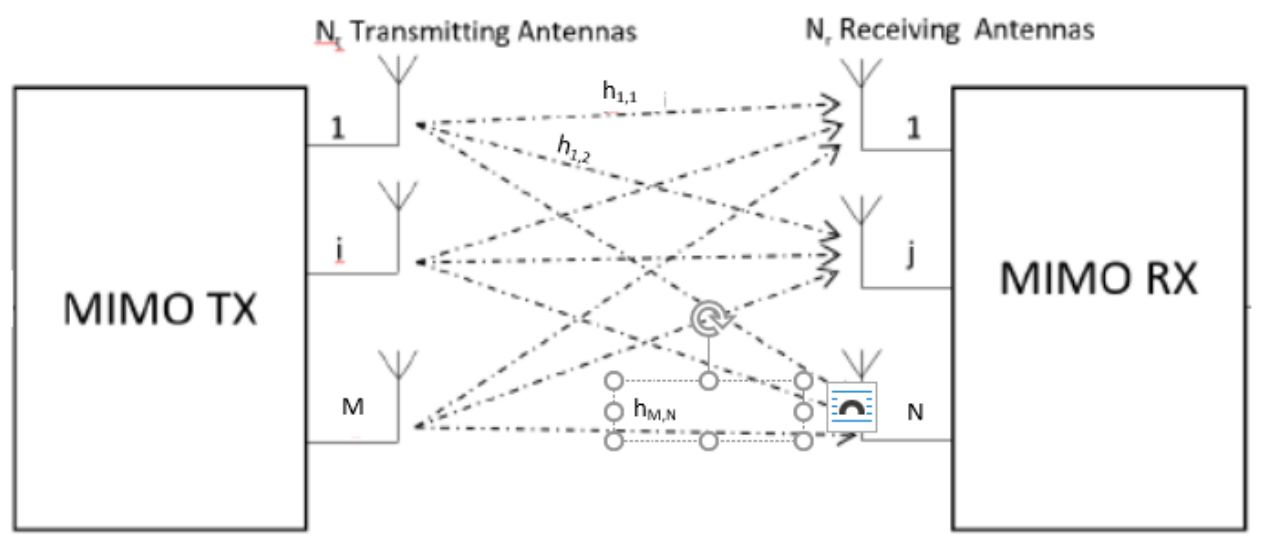

Figure 2.2: MIMO wireless communication system.

system and to attain relabel and efficient algorithms in MIMO system for efficient utilization of MIMO benefits a rigid representation of MIMO channel should be assigned. The MIMO channel for a wireless communication system with M transmit, and $\mathrm{N}$ receive antenna can be represented mathematically by [29]:

$$
\mathbf{H}=\left[\begin{array}{ccccc}
\mathbf{h}_{11} & \cdots & \cdots & \cdots & \mathbf{h}_{1 M} \\
\mathbf{h}_{21} & \ddots & \ddots & \ddots & \mathbf{h}_{21} \\
\vdots & \ddots & \ddots & \ddots & \vdots \\
\vdots & \ddots & \ddots & \ddots & \vdots \\
\mathbf{h}_{1 M} & \ddots & \ddots & \ddots & \mathbf{h}_{N M}
\end{array}\right]
$$

Where $\mathbf{h}_{n m}$ represent the channel gain between the mth transmute antenna and nth receive antenna as shown in figure 2.2. The received signal vector at the receiver is given by: 


$$
\mathbf{y}=\mathbf{H x}+n
$$

Where $\mathbf{y} \in C^{N \times 1}$ is the received signal vector at the receiver, $\mathbf{H} \in \mathbb{C}^{N \times M}$ is is the channel matrix, and $\mathbf{n} \in C^{N \times 1}$ is the noise vector.

Because of its important of CSI a lot of methods and algorithms has been investigated to estimate it and the most common method to estimate the CSI are: 1) Training based CSI acquisition:

In this method the transmitter and/or receiver send pilot signal to the receiver and then receive a feedback from the receiver. This method is used in conventional wireless network and, in the secondary network in wireless cognitive network. The method cannot be used to estimate the CSI between the secondary and primary user in cognitive network if there is no cooperation between the primary and secondary user. Different algorithms have been invoked using this method [30-34].

2) Blind CSI acquisition:

In this method the secondary user in cognitive network detects the control signal send by the primary user and estimate some related CSI between the secondary and primary user. This method is applicable if the primary user performs CDMA, HSDPA, Wi-Fi and LTE-A. as a part of its operations [35,36].

The CSI can be estimated deterministically or statistically. The deterministic estimation is applicable if the measurement or estimation time is small as compared with channel coherence time $[38,39,81]$. The deterministic estimation is suitable for 
slow fading channel. In fast fading channel the statistical CSI estimation are used where the measurement over long time is averaged [40]

\subsection{CSI Uncertainty}

Due to channel estimation error, limited feedback, and/or the instantaneous nature of wireless channel, the estimated CSI is subject to error [41-43] this led to inefficient gain of MIMO benefit. The error in CSI can be mathematically represented as:

$$
\mathbf{H}=\widehat{\mathbf{H}}+\Delta \mathbf{H}
$$

Where $\mathbf{H}$ is the actual CSI, $\widehat{\mathbf{H}}$ is the estimated CSI, and $\boldsymbol{\Delta} \mathbf{H}$ is the error in CSI. The error or which is usually called the uncertainty, can be modeled scholastically or deterministically. In stochastic representation the designer seeks to improve the outage probability and eliminate the uncertainty effect through some sophisticated mathematical process. In deterministic model the quality of service should be improved either by maintaining specific signal to noise ratio at the receiver side and minimize the transmitted power by the transmitter or improve the signal to interference noise ratio at the receiver for some limited transmitted power by the transmitter. In deterministic models which of concern in this work different mathematical set are available such as:

1) Polyhedron set.

2) Ellipsoid, spectral and Frobenius norm sets. 
3)Schatten norm and unitary-invariant sets.

In this work we consider the deterministic model bounded by the Frobenius norm mathematical set.

When the channel subject to error bounded by Frobenius norm this mean that the CSI can be represented by:

$$
\mathbf{H}=\widehat{\mathbf{H}}+\Delta \mathbf{H}
$$

$$
\|\mathbf{H}\|_{F} \leq \epsilon
$$

Where $\epsilon$ represent the error in value in CSI and must be limited to be not more than one.

\subsection{Cognitive Radio Network}

The Federal Communication Commission (FCC) maintain that $70 \%$ of the licensed spectrum is underutilized [44] which are called spectrum holes or white spaces as shown in figure 2.3. The underutilized spectrum can be a good source to resolve the problem of frequencies scarcity in wireless communication which is a result of high advances in wireless communication technologies that require high data rate for the new multimedia applications. The FCC suggests three solutions to regulate utilizing these spectrum holes. which are: 


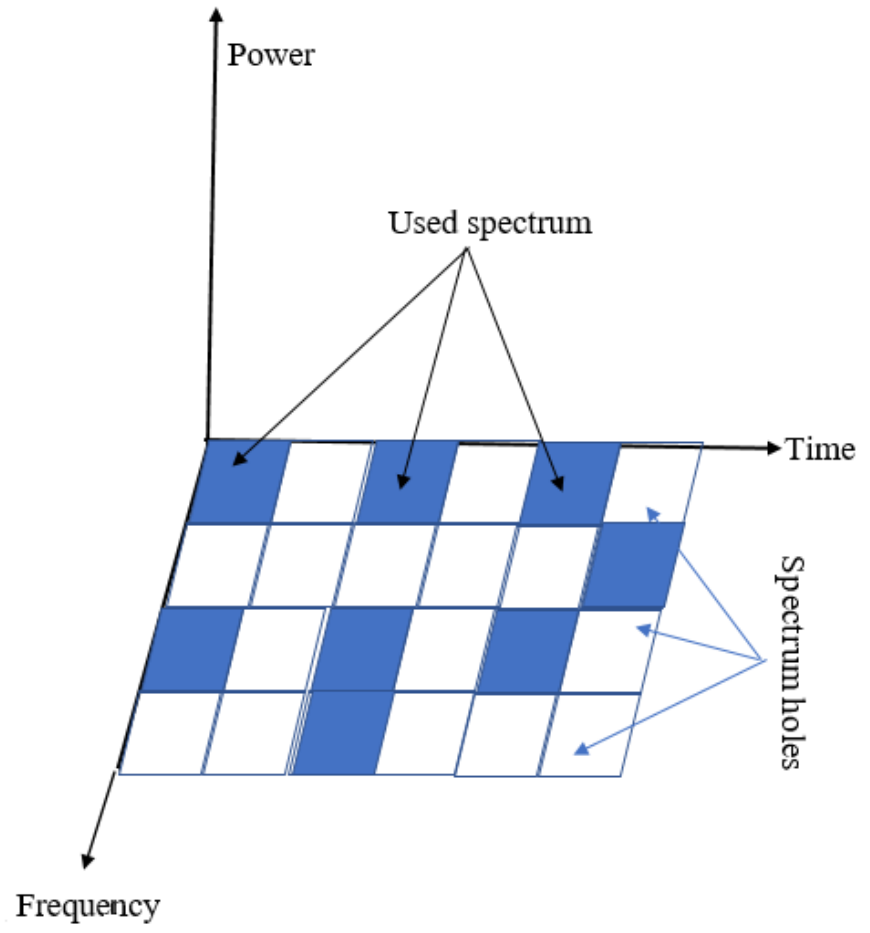

Figure 2.3: Utilization of frequency resource in wireless communication.

1) Spectrum reallocation [45].

2) Spectrum leasing [46].

3) Spectrum sharing [47].

In spectrum sharing the licensed frequencies are assigned to the primary users but these users do not use all frequencies band at sometimes thus the underutilized frequencies are resigned to the secondary users through dynamic spectrum allocation(DSA)technique. This type of frequency sharing allows different heterogeneous network to cooperate and work together without degrading the quality of service of 


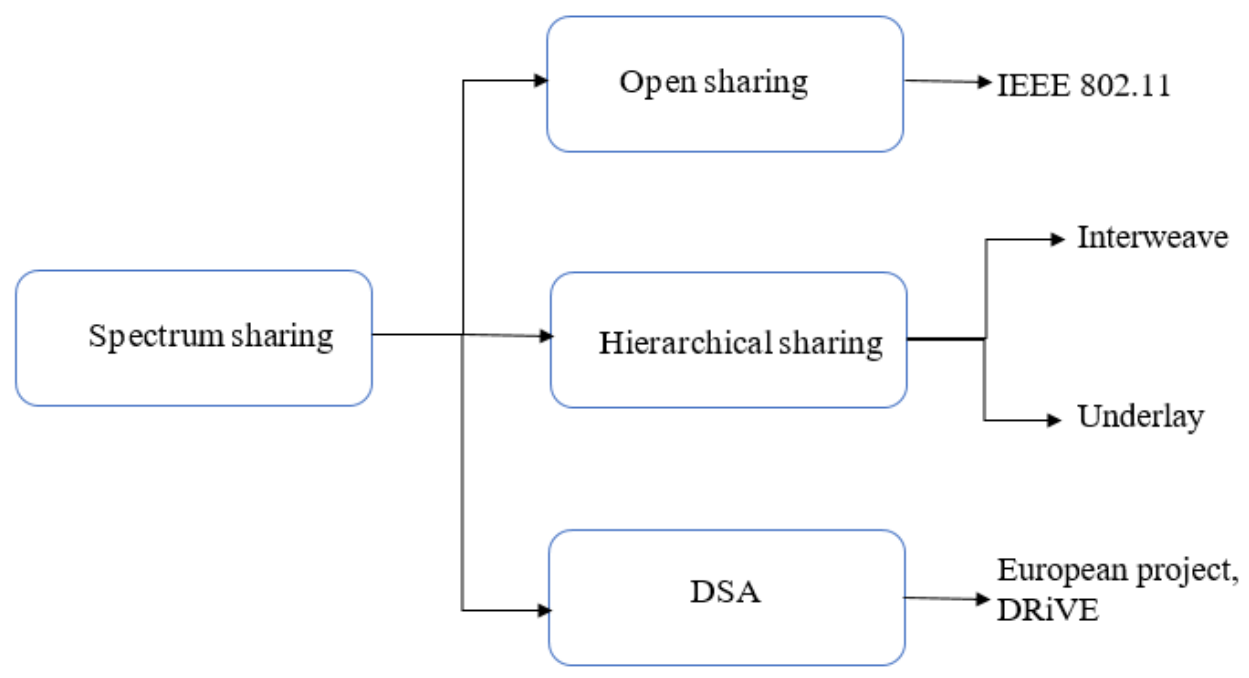

Figure 2.4: Type of sharing technique.

any user. In this work we considered the spectrum sharing method which divided into three categories as shown in figure 2.4.

In the hierarchical which is considered in this work the primary user has the highest priority to use the licensed frequency and the secondary user assigned the lowest. The amount of available CSI at the secondary user has an important strategies of the utilizing the spectrum holes by the secondary users. Also, the CSI has an effect on categorizing the hierarchical sharing into two modes, the interweave (overlay) mode and underlay mode. 


\subsubsection{Interweave Cognitive Network}

In this mode, the cognitive network sensing the spectrum holes and using some sophisticated signal processing algorithm to utilize one of the holes. In this mode, the network continuously monitors the primary user to release the used frequency to the primary user when it restart to use it. Different works have been achieved to design the suitable algorithms for spectrum sensing in a cognitive wireless network.

\subsubsection{Underlay Cognitive Network}

In this mode of hierarchical sharing network, the secondary user share the same frequency used by the primary user instantaneously under rule that the interferences from the secondary user to the primary user are under small predefined level. In this mode the secondary user must know the interference CSI between the secondary user and the primary user in order not to affect the quality of service of the primary user. Using MIMO technique can significantly improve hierarchical sharing in wireless network. Specifically, applying MIMO at the secondary user in overlay cognitive networks attain rigid spectrum sensing by investing the spatial diversity. Also, applying MIMO at the primary user does not need to know the CSI between the primary and secondary users. In an underlay cognitive network applying MIMO at the secondary user can significantly reduce the secondary user's interference power to the primary user. In an underlay cognitive network, the secondary user needs to estimate the CSI from the secondary transmitter to the secondary receiver and the CSI from the secondary transmitter to the primary receiver if the primary transmitter resides in the other network. 


\title{
2.5 Beamforming
}

As we maintain previously the wireless communication signals suffer from propagation affects that my degrade the received signal, these affects can be categorized to.

\author{
1) Large-scale fading \\ a- Shading. \\ b- Path loss. \\ 2) Multipath.
}

In $4 \mathrm{G}$ and up the MIMO technique is utilized by using multiple antenna at the transmitter and/or receiver to mitigate the effects of small-scale fading by combat the multipath signal through diversity multiplexing called beamforming. The beamforming improves the rate and received power at the receiver by concentrating the transmitted power of desired signal toward the desired receiver and reduce the power of unwanted signal toward this receiver. The beamforming can be classified into different types according to the number of receivers, direction of beamformed signal, type of communication networks, and the degree of availability of CSI at the transmitter as shown in figure 2.5 . 


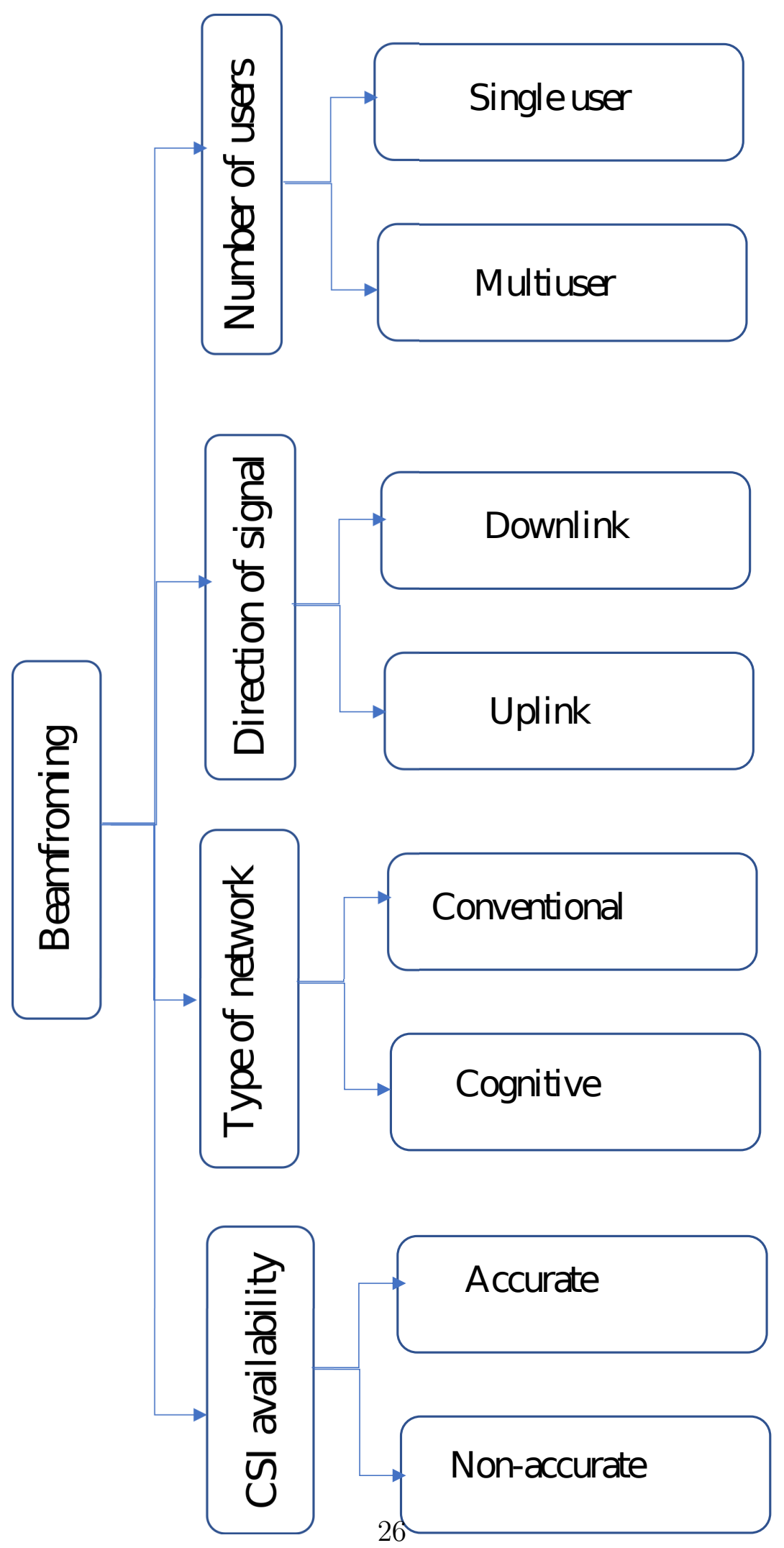

Figure 2.5: Category of different beamforming in wireless communication system. 


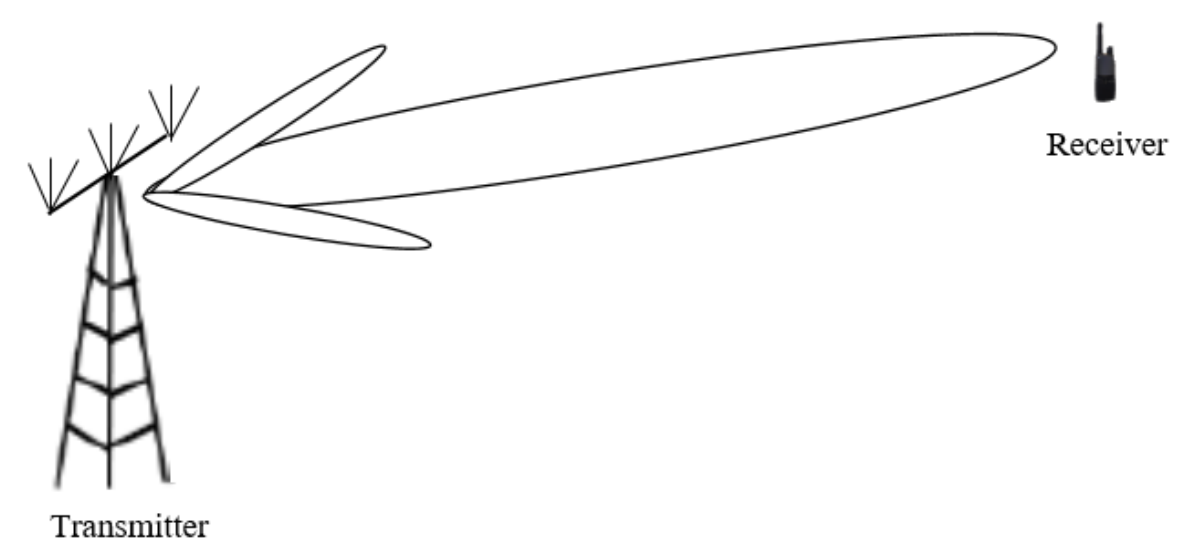

Figure 2.6: Single user baemforming IN MIMO system.

\subsubsection{Number of user}

The number of users has very important effect on obtaining beamforming vector(s) where in a single user the transmitter send single beamforming signal as shown in figure 2.6 the optimal beamforming vector can be obtained by simply extract the principle Eigenvector of the CSI between the transmitter and the receiver.

The received signal power at the receiver is given by:

$$
\mathbf{w}^{H} \mathbf{H w}
$$

where $\mathbf{w}$ is the beamforming vector and $\mathbf{H}$ is the CSI at the side doing beamforming. If the number of transmit antenna exceed the number of receive antenna in the wireless communication system, then the transmitter can perform multi-beamforming and the number of beamforming vectors that can be transmitted equal to the difference between the number of antenna at the transmitter and receiver as shown in figure 2.7. The beamforming vector can be extracted by solving one of the following 


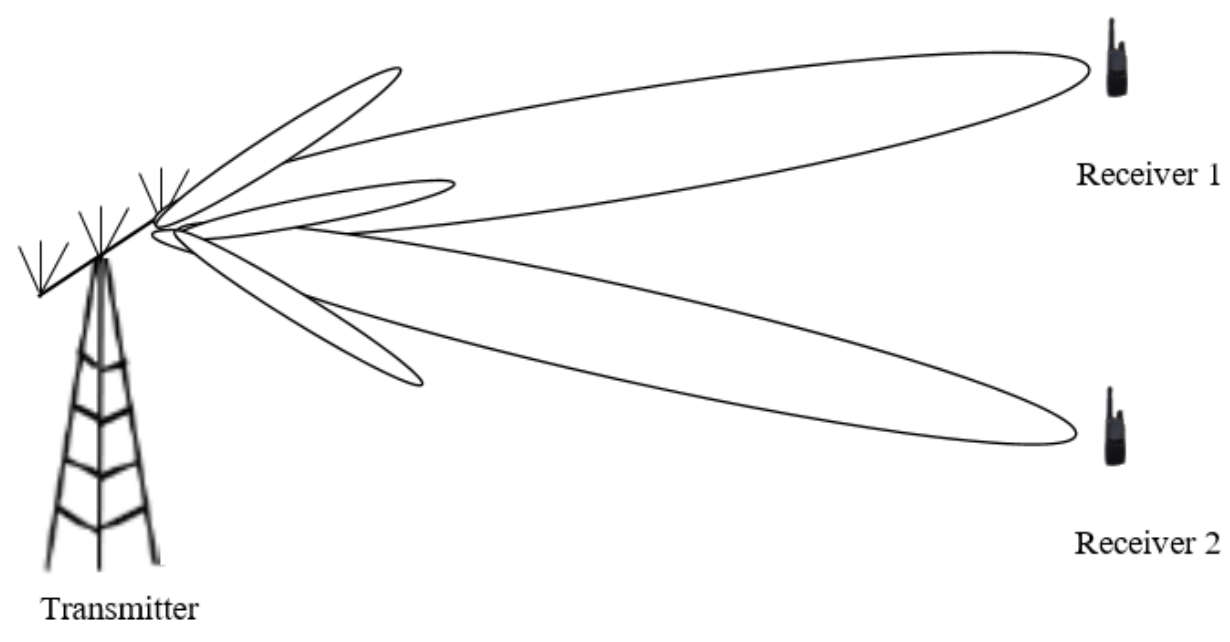

Figure 2.7: multiuser baemforming IN MIMO system.

optimization problem:

$$
\begin{aligned}
& \max _{\{\mathbf{w}\}_{k=1}^{K}} \min \frac{\mathbf{w}_{k}^{H} \mathbf{R}_{m} \mathbf{w}_{k}}{\sum_{i=1, i \neq k}^{K} \mathbf{w}_{i}^{H} \mathbf{R}_{m} \mathbf{w}_{i}+\sigma_{m}^{2}} \\
& \text { s.t. } \sum_{i=1}^{K}\left\|\mathbf{w}_{i}\right\|^{2} \leq P_{b}
\end{aligned}
$$

where $\mathrm{K}$ represent the number of users and $P_{b}$ is the total transmitted power.or using:

$$
\begin{gathered}
\min _{\{\mathbf{w}\}_{k=1}^{K}} \sum_{i=1}^{K}\left\|\mathbf{w}_{i}\right\|^{2} \\
\text { s.t. } \frac{\mathbf{w}_{k}^{H} \mathbf{R}_{m} \mathbf{w}_{k}}{\sum_{i=1, i \neq k}^{K} \mathbf{w}_{i}^{H} \mathbf{R}_{m} \mathbf{w}_{i}+\sigma_{m}^{2}} \geq \gamma
\end{gathered}
$$

where $\gamma$ is the minimum allowable signal to interference noise ratio at the receiver. 


\subsection{2 direction}

According to the direction of signal the beamforming vector is divided into two type, the uplink, and the downlink. The uplink beamforming has gain many attention in wireless communication with the multiantenna development $[48,49]$ due to the grate achievement in term of data rate and signal power. The beamforing vector is calibrated in uplink scenario such that the direction of co-channel interference are paced in the null space as well as the multipath signal and the gain is improved in the direction of the signal of interest. the uplink beamforming receive a lot of attention in many area such as sonar system, biomedical system.....etc. The downlink beamforming which widely applied in LTE, and WiMAX [50,51], where the signal is directed toward desired user and weakened in other direction as in uplink beamforming but the main difference is the downlink beamforming is used to direct the communication signal(s) to single or multiuser by using multiple beamforming vectors and thus lead to improve the quality of service at each user. The main different between the uplink and downlink beamforming is that in downlink beamforming the beamforming vectors are designed jointly while in uplink scenario the beamforming vectors is designed separately in each user and thus the beamforming vector for specific signal has less effect on other signal in downlink beamforming while in uplink beamforming each signal has an effect on the other signal because the beamforming vectors are designed separately.

\subsubsection{Type Of Communication Network}

Equation (2.8) or (2.9) is typically used to extract the beamforming vectors in conventional wireless network, in cognitive wireless network the case must be modified. 
As we maintain previously there are two type of cognitive wireless communication the overlay and the underlay network. In overlay, the secondary user starts to communicate when the primary user is salient or absence and thus no modifications are required on equations (2.8) and (2.9). On the other hand, in underlay cognitive network the secondary user communicate wither the primary user is active or not and thus, the secondary user must transmit at a power that in the level not affect the primary user. This can be achieved by adding more constraint to the (2.8) and (2.9) this constraint is only represent the level of power that affect from the secondary user to the primary user $[52,53]$

\subsubsection{CSI Accuracy}

As we maintain previously, different techniques have been used to estimate CSI, and different algorithms were invoked for each technique. for an accurate CSI estimation equation (2.8) or (2.9) can be directly used to determines the beamforming vectors. But due to the practical limitations such as the dynamic characteristics of channel(estimation time larger than the coherence time), limited feedback and/or estimation error the gathered CSI is subjected to error that can be modeled using different mathematical models. In the case that the estimated CSI is not accurate equation(2.8) and (2.9) cannot applied directly to determine the beamforming vector; thus in beamforming technique two type of solution were developed which are: 1) Sub-optimal solution: in this solution the beamforming vectors are extracted under worst case condition (lowest quality of service at the receiver) but with less computational complexity. 2) Robust solution: in this solution the beamforming vectors are extracted robustly by mitigating the effect of CSI error though some sophisticated 
math manipulations. In this solution, the generated beamforming vector can achieve better Quality of service at the receiver but the method requires high computational complexity than the sub-optimal method.

\subsection{Convex Optimization}

Convex optimization is a mathematical topics because of integration of three mathematical subjects:

1) Convex analysis $[54,55]$.

2) Optimization $[56,57]$.

3) Numerical computation $[58,58]$

Now it consider as an important tool in Engineering where it can be used to find efficient and reliable solution for large problems.

The formal problem in convex optimization takes the form:

$$
\begin{gathered}
\underset{x}{\operatorname{minimize} . \mathbf{f}_{0}(x)} \\
\text { s.t. } \mathbf{f}_{i}(x) \leq 0 \forall i=1, \ldots, m \\
\mathbf{f}_{k}(x)=0 \forall k=1, \ldots, n
\end{gathered}
$$

Where $\mathrm{x}$ is the variable vector need to find, $\mathbf{f}_{0}(x)$ is the objective function and $\mathbf{f}_{i}(x) \leq 0 \forall i=1, \ldots, m$ and $\mathbf{f}_{k}(x)=0 \forall k=1, \ldots, n$ is the inequality and equality constraints.

If the objective function and the inequality constraints is convex function and the equality constraints is affine then the optimization problem is called convex opti- 

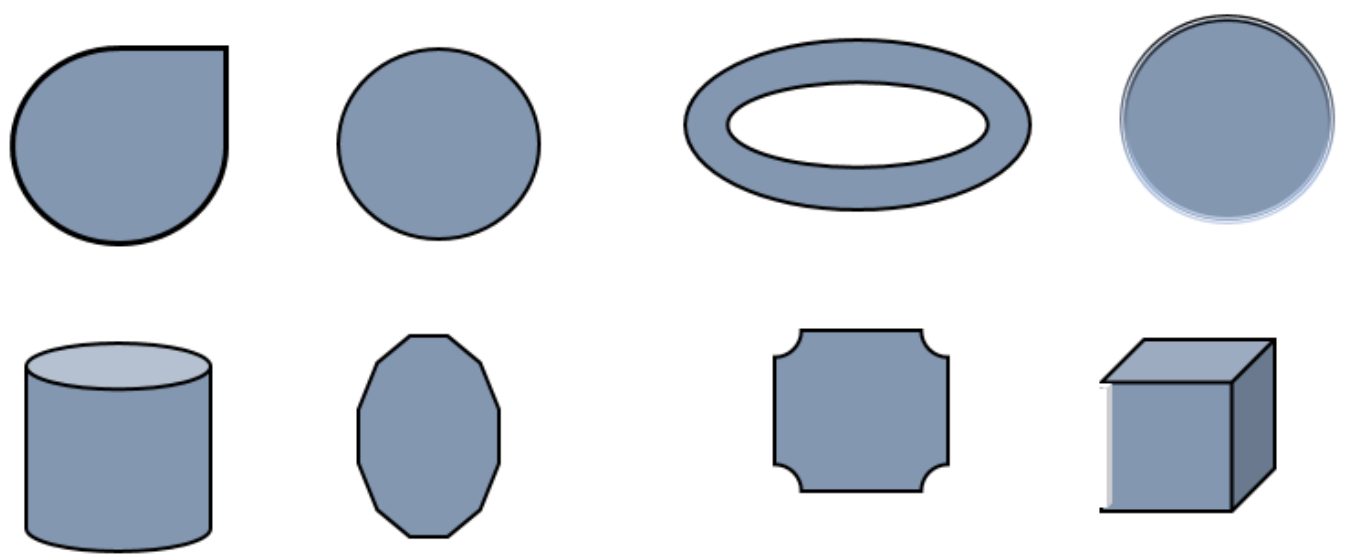

a-Convex set

b-Non-convex set

Figure 2.8: Convex and Non-convex set.

mization problem in condition that the set in which the optimization variable lie is convex set.

The set is convex if a line segment whiten it contain all the points of the line as shown in figure 2.8. for more details see [59]

\subsection{Successive Convex Approximation}

The function is convex if it satisfy the following criteria:

$$
\mathbf{f}\left(\lambda \mathbf{x}_{1}+(1-\lambda) \mathbf{x}_{2}\right) \leq \lambda \mathbf{f}\left(\mathbf{x}_{1}\right)+(1-\lambda) \mathbf{f}\left(\mathbf{x}_{2}\right)
$$


Where $\lambda \in[0,1]$ as shown in figure

A function can be concave, and if $\mathrm{f} 1$ is concave, then $-\mathrm{f} 1$ is convex for more details see $[60]$

\subsection{Lagrange duality}

Lagrange duality is a method to solve an optimization problem by finding a solution to an equivalent problem. The first problem is known as the primal problem, while the second is called the dual problem [56]. This procedure is follows when there is a difficulty in solving the primal problem.

$$
\begin{gathered}
\operatorname{minimize} . \mathbf{f}_{0}(x) \\
\text { s.t. } \mathbf{f}_{i}(x) \leq 0 \\
\forall i \in(1, \ldots, m) \\
\mathbf{f}_{k}(x)=0 \\
\forall k \in(1, \ldots, n)
\end{gathered}
$$

Where $\mathbf{f}_{0}(x)$ is the objective function, $\mathbf{f}_{i}(x)$ is the inequality constraint , and $\mathbf{f}_{k}(x)$ is the quality constraint. The above is the primal problem, and the equivalent dual problem is:

$$
\mathrm{d}(x, \lambda, \mu)=\inf \left(\mathbf{f}_{0}(x)+\sum_{i=1}^{m} \lambda_{i} \mathbf{f}_{i}(x)+\sum_{k=1}^{n} \lambda_{i} \mathbf{f}_{k}(x)\right)
$$

Where $\lambda_{i} \forall i \in(1, \ldots, m)$ and $\mu_{k} \forall k \in(1, \ldots, n)$ are the Lagrange multiplier associated with inequality and equality constraints, respectively 
The resultant dual function is always convex, even if the original primal function is not convex. Depending on the resultant optimal value from the dual function(d) and the actual optimal value of the primal function(p) there are two type of duality [61]: 1) Strong duality.

2) Weak duality.

These types of duality depend on the duality gape between the dual value(d) and primal value(p). If the duality gap is greater than or equal to zero (i.e. the objective vale of the dual problem is less than or equal the objective value of the primal problem), then the problem has weak duality; otherwise, if the objective value of the dual and primal problems are the same, then the problem has strong duality as shown in figure 2.9 .

\subsection{Successive Convex Approximation}

Many problems in engineering are formulated as a non-convex where the objective function is non-convex, and the constraints are convex or non-convex but, most of them obtaining the global solution are computationally expense since they have no closed form solutions are available. The goal is to find a method that can be proved to be converge and easy to implement. The successive convex approximation (SCA) method is the powerful method to solve such problem [62].

The militarization-minimization framework is special example of SCA method and can be considered as the base to design the SCA method. In this problem the general is to consider the following nonconvex optimization problem. 


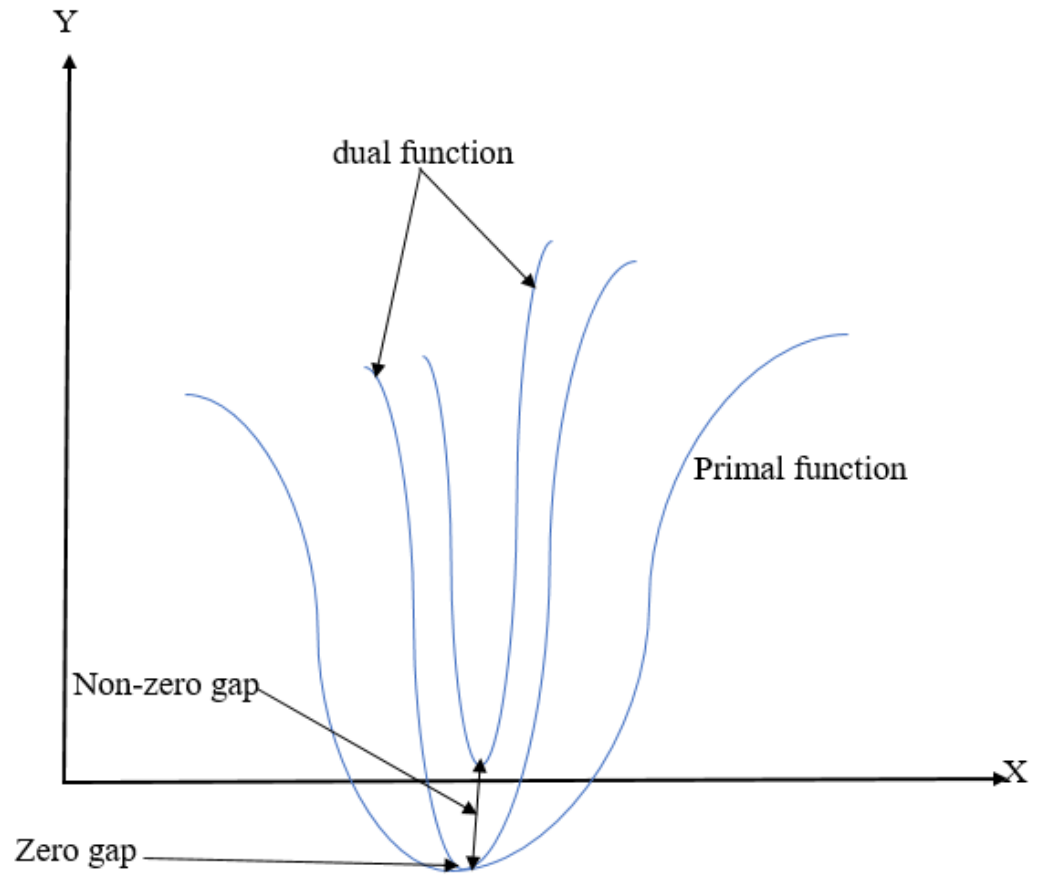

Figure 2.9: strong and weak duality explanation. 


$$
\begin{gathered}
\underset{\mathbf{x}}{\operatorname{minimize}} \mathbf{f}_{0}(x)+\mathbf{g}_{0}(x) \\
\text { s.t. } \mathbf{f}_{i}(x)+\mathbf{g}_{i}(x) \\
\forall i \in(1, \ldots, m)
\end{gathered}
$$

The functionf $\mathbf{f}_{i}(x)$ is non-convex while $\mathbf{g}_{i}(x)$ is convex. The above problem can be solved using the following algorithm:

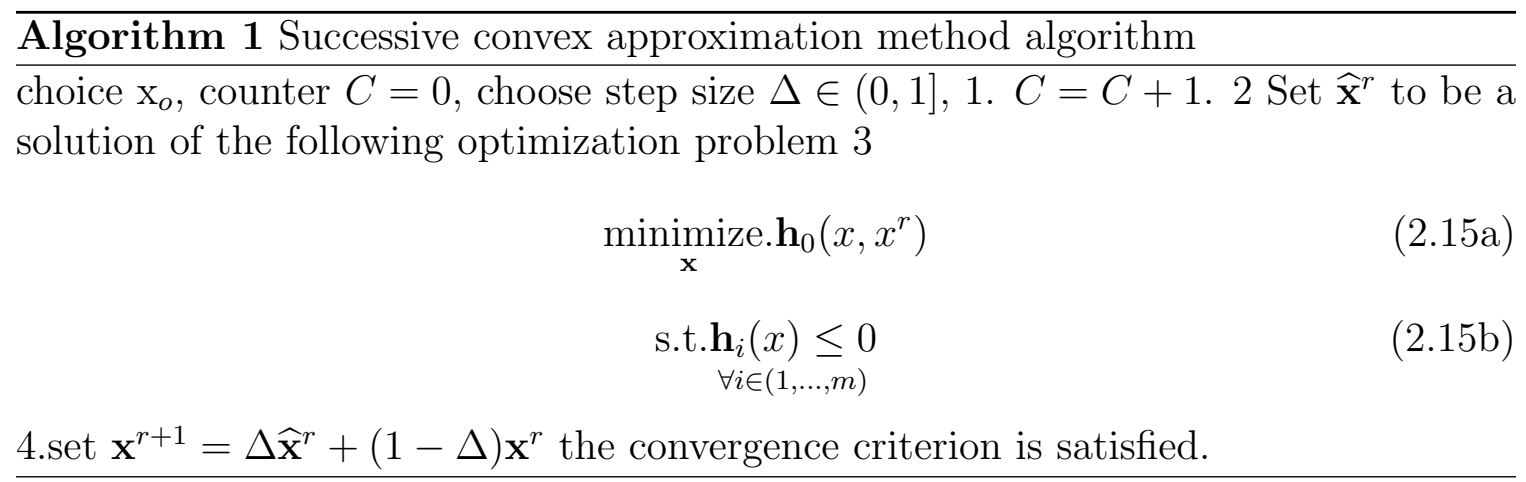

For more details about the assumption that need to be satisfied by the above algorithm please refer to [63].

The way the algorithm converge can be shown in figure 2.10

\subsection{0 bisection search method}

The synonym of this method is binary search method, interval halving, or dichotomy [64]. The method is numerical method to find the roots of continuous non-linear function by splitting the interval of the function in half iteratively, as shown in figure 2.11. the main advantages of this method are: 


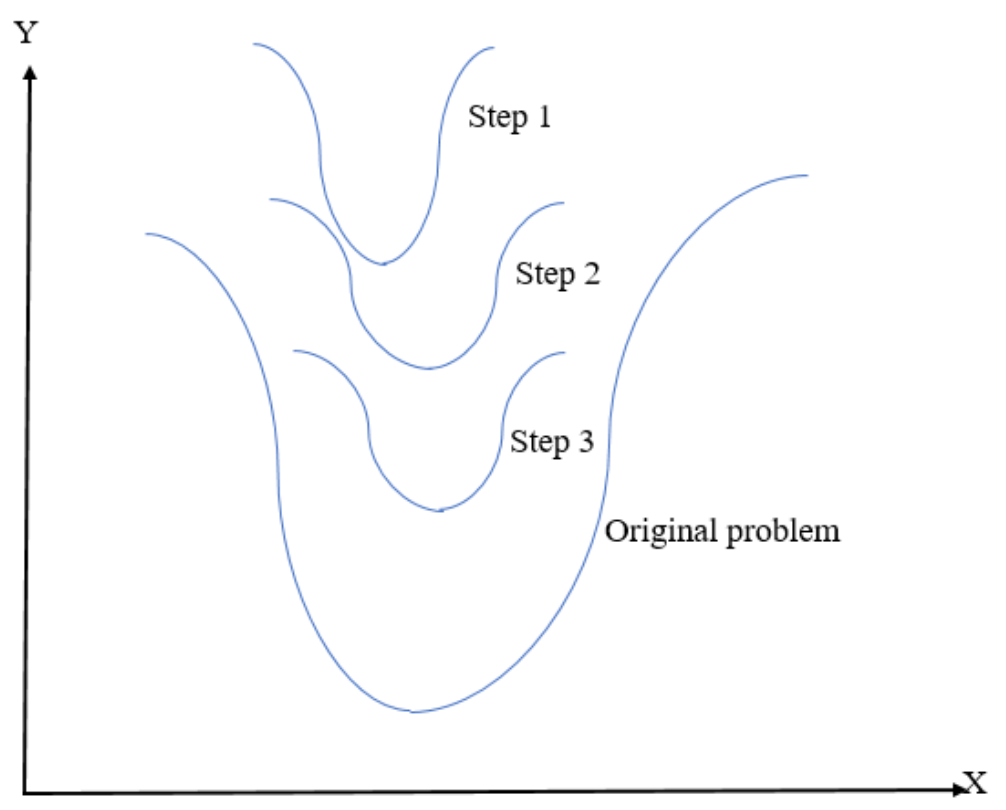

Figure 2.10: convergence of successive convex approximation method.

1) It has high speed as compared with other numerical method because it halves the interval each time of iteration.

2) It has higher accuracy in determining the roots of the polynomial as compared with incremental method.

On the other side, the disadvantages of this method are: 1) If the roots' function is determined are of complex values, then the method fails to estimate these roots.

2) If the roots of a function to be determined lies on the axis the the method fail to determine these roots.

3)If the function to which the roots have to be determine is a singularity function, the bisection search method fall to estimate the exact or approximate roots.

The method divides the intervals of a polynomial iteratively to extract the roots, the final intervals should shrine to zero to estimate the exact roots, but this means 


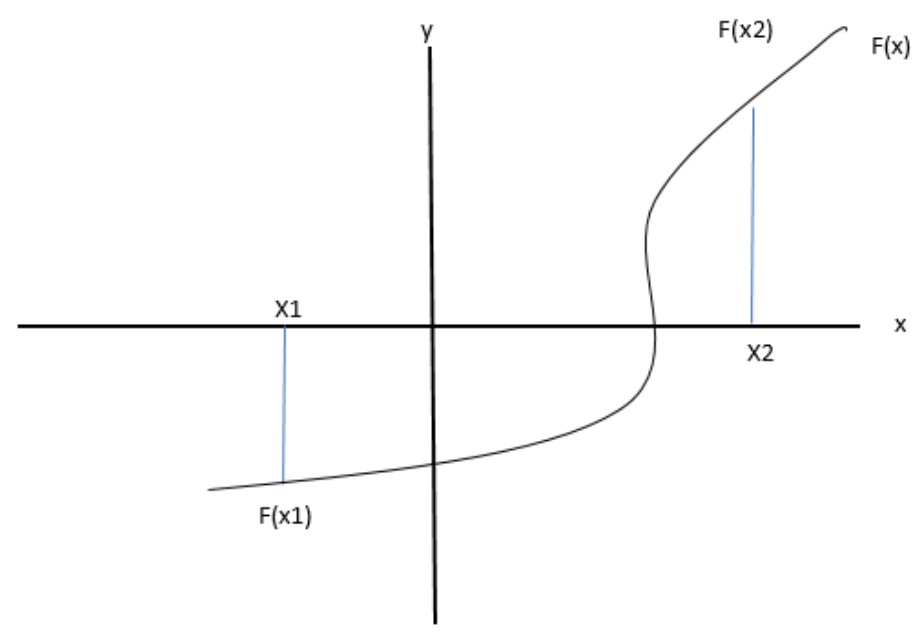

Figure 2.11: Bisection search method starting interval.

that the number of iteration must go infinite. To avoid infinite number of iterations, the iteration must be stopped when the interval reaches a small predefined value, as shown in figure 2.12 or the number of iteration reach to maximum predefined value. the method work as follows [65]:

1) let we have function $\mathbf{F}(\mathbf{x})$ as shown in figure below: 2)Chose two value $\mathbf{F}\left(\mathbf{x}_{\mathbf{1}}\right)$ and $\mathbf{F}\left(\mathbf{x}_{\mathbf{2}}\right)$ such that the gap between $x_{1}$ and $x_{2}$ are large enough.

3) Determine $F\left(x_{3}\right)$ such that:

$$
x_{3}=\frac{x_{1}+x 2}{2}
$$

4) If $F\left(x_{3}\right)$ is equal to zero, then the method is fail to determine the roots it must stooped or restarted again by going to step 2 otherwise:

a) If $F\left(x_{3}\right)<F\left(x_{2}\right)$ then $x_{2}=x 3$ 


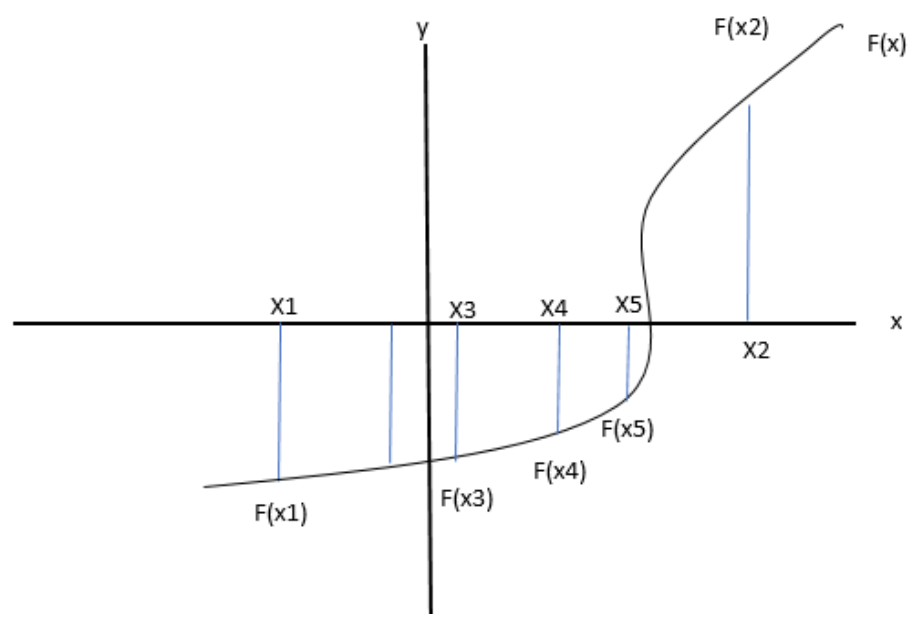

Figure 2.12: Bisection search end interval.

b)If $x 1<x 3$ then $x_{1}=x 3$

5) If $F\left(x_{3}\right)$ is less than or equal the predefined error or the number of iteration reach the maximum number of iteration stop the algorithm or go to step 3 and repeat. 


\section{Chapter 3}

\section{Worst Case Fair Beamforming for Multiple Multicast Groups in Multicell Networks}

\subsection{Introduction}

The design problem associated with robust downlink beamforming in multicast, multigroup, multicell wireless systems is addressed. The channel state information (CSI) of users is assumed to be imperfect and the uncertainty of CSI is modeled using the Frobenius norm. The objective is to optimize the signal to interference plus noise ratio over all users with a constraint on the maximum total transmitted power. This was achieved through a robust solution using the successive convex approximation (SCA) method. The beamforming problem is treated as a bi-convex problem which is solved using the iterate-alternative convex technique. Here the CSI uncertainty is addressed using a convex package through the non-monotone spectral projected 
gradient (NMSPG) method and the beamforming vector is extracted using the SCA method. Also, we offer the required condition to extract the beamform vector using SCA method through suboptimal solution that always addressed before using different beamforming methods. Our simulation results examine all proposed system parameters in order to show convergence and feasibility of our solution. We also compare our solution with suboptimal solution and the QOS method for imperfect CSI in downlink beamforming. Numerical results show that the robust solution achieves the best power efficiency for a practical solutions.

\subsection{Background}

Wireless cellular communication requires high data rates in order to meet some internet application requirements, such as, video streaming, where the same data is simultaneously sent to multiple users. The multiple input multiple output (MIMO) [66] is a well established technique that can improve physical layers in wireless communication by increasing the throughput and reliability of the system while controlling the quality of service (QOS) at the end user by reducing the interference of multiusers. An advanced MIMO physical layer standard in cellular communication systems such as Long-Term Evolution(LTE) and the advanced LTE can be easily implemented using the multicast, multigroup, multcell, beamforming techniques [67-71] and has become an important area of research in recent years.

Further improvement for downlink beamforming systems using the MIMO technique can be achieved if the channel state information (CSI) is available at the trans-

mitter in the form of instantaneous CSI or covariance based CSI. Much research 
has been conducted to obtain the CSI in MIMO systems such as $[72,73]$. However, due to several practical limitations in wireless communication, such as the dynamic characteristics of channels [74], limited feedback [75,76] and/or estimation error [77], the estimated CSI contains some error and thus the uncertainty in the observed CSI should be taken into consideration. This line of research has been addressed in [78-82] and the references therein.

The uncertainty in the CSI can be modeled stochastically or deterministically. In the stochastic model, the instantaneous value of the CSI is not known, and the CSI is modeled as a random quantity whose mean and/or covariance at the transmitter is assumed to be known. In the deterministic model the CSI uncertainty belongs to a set of known shape and size. This chapter considers the deterministic model for representing the estimation error in the CSI.

schad2012max,hanif2010sinr Given the importance of downlink beamforming in MIMO systems, various beamforming designs have been developed in the literature for perfect CSI modeling [71,83-85]. Some designs depend on the type of wireless network (either conventional or cognitive), while other depend on the form of the CSI used to formulate the beamforming problem (either instantaneous CSI or covariance based CSI), moreover other designs depend on the method used to solve the beamforming problem (such as semidefinite relaxation(SDR) or SCA method). Accordingly, different robust downlink beamforming solutions have been investigated to solve the issue of uncertainty in the CSI whether instantaneous based or covariance based. In this chapter we will design a downlink beamform vector for a MISO system in a conventional network using the SCA method with uncertainty in covariance based CSI. 
In the literature there are two main types of solutions for CSI uncertainty in downlink beamforming. The authors in $[86,87]$ provided a heuristic solution for imperfect CSI in downlink beamforming, under the worst case conditions, however, this solution gave poor results in terms of power efficiency. A second approach leads to a robust solution for the problem by considering a closed form expression for the CSI uncertainty. In particular, the authors in [76,88-90] consider a robust solution when imperfect instantaneous based CSI is used while the authors in [91-94] consider the solution for the case of covariance based CSI. This second approach outperforms the approach in $[86,87]$. The authors in $[76,94]$ solve the problem in a conventional network and the authors in $[85,95]$ solve it for a cognitive network. Another approach is to either use the well known S-lemma [76] or to obtain an equivalent formulation utilizing Lagrangian duality [94]. All of the above solutions use the SDR method to tackle the problem of CSI uncertainty.

The drawback of the SDR method is that it removes the rank constraint to relax the optimization problem, and hence, an additional approximation step, called reformulation, is needed to extract the beamforming vectors. The authors in [?]robust found a robust solution using the rank two beamforming technique through the SDR method but still needed the additional reformulation step when the resulting beamform matrix is of rank greater than two. Therefore, the main problem associated with the SDR method is the rank approximation. The authors in [71] found a solution for the rank problem in the beamforming technique by utilizing a general rank beamforming technique. The main problem associated with this method is that 
owing to the transmission of multiple samples instantaneously using the Alamouti code, the rate of the system is reduced to about $75 \%$. Another drawback of the SDR technique, as compared with the SCA technique, is its computational cost, since the former involves high computational complexity for the same beamforming technique, especially when the system dimension increases.

chapter considers the deterministic CSI model and obtains a beamforming vector under the worst case conditions. The covariance CSI matrix is determined assuming slow fading channel and the uncertainty set is considered to be spherically bounded with respect to the Frobenius norm. The beamforming technique used is the max-min fair download beamforming where the minimum SINR is maximized over all receivers in all groups that receive the beamform signal from multi base stations. Finally the method used to solve the optimization problem is the SCA method instead of the SDR method.

The main contribution of our work is that we obtain a robust solution for the beamforming vector by following the SCA method, where we derive the beamforming vectors directly and avoid using the SDR method, following the previous work [76,86-95] since the SDR method gives the solution by either ignoring the rank constraint (which requires the additional approximation step of reformulation) or by using a general rank beamforming technique, which reduces the rate of the system. We find the worst case CSI by modifying theorem 1 in [96], where the author in [96] found a solution of $\mathbf{X}$ in the problem $(\operatorname{Max} \operatorname{Tr}(\mathbf{X Y}))$ when $\mathbf{Y}$ is known and positive (semi)definite. Herein we a find solution $\mathbf{X}$ of the problem $(\operatorname{Max} \operatorname{Tr}(\mathbf{X Y}))$, when $\mathbf{Y}$ is known and Hermitian. Moreover, our solution is shown to be more power efficient than the sub-optimal so- 
lution suggested by the authors in $[86,87]$, where they assume the worst case solution for the SINR, and the solution suggested by the authors in [94] that found a closed form solution for the CSI uncertainty via Lagrangian duality.

The remainder of this chapter is organized as follows: Section II introduces the multicast, multigroup, multicell beamforming problem under the max-min fair (MMF) scenario and then shows the non-robust associated problem formulation. In addition, we describe the uncertainty of the parameters that relate the instantaneous CSI uncertainty radius with covariance CSI uncertainty. In Section III, we solve the uncertainty problem under the worst case scenario and present the required conditions that demonstrate convergence to the solution. Section IV describes the proposed solution where the theorem in [96] is modified to best fit SCA which has been proposed to solve the MMF beamforming problem with perfect CSI in [85]. In Section $\mathrm{V}$, different simulation results are presented to show the convergence and feasibility of our solution under all system parameters.

In our notation, uppercase and lowercase bold letters represent matrices and vectors, respectively, throughout the chapter; $\mathbf{E}[X]$ denotes the statistical expectation of the random matrix $\mathbf{X} ; \mathbf{X} \succeq 0$ denote a PSD matrix, $\mathbb{C}^{m \times n}$ denotes a complex $m \times n$ matrix, $\operatorname{diag}\left\{\mathbf{e}_{b}\right\}$ is a $K \times K$ zero matrix whose (b,b) element is one; |.|, $\|\mathbf{X}\|_{F}$ are the absolute value and the Frobenius norm of the matrix $\mathbf{X} ;(.)^{H}$ denotes Hermitian transpose of vector or matrix and $(\mathbf{X})^{+}$the Hermitian matrix $\mathbf{X}$ converted to a PSD matrix; $\mathbf{R}_{m}, \mathbf{w}_{i}$ are the $m^{\text {th }}$ covariance matrix and $i^{t h}$ beamform vector; $\mathbf{I}_{N}, \Lambda, \lambda_{n}(X)$ denote the $N \times N$ identity matrix, eigenvalue matrix and $n^{\text {th }}$ eigenvalue of the matrix 
$\mathrm{X}$ respectively; finally, $\otimes$ stands for the Kronecker product.

\subsection{System model and Problem Formulation}

\subsubsection{System Model}

Consider a multicell wireless scenario, consisting of B base stations each with $N$ transmit antennas served synchronously by $M$ single antenna receivers. All the receivers are divided into $K$ multicasting groups $\left\{G_{1}, \ldots, G_{K}\right\}$. The receivers in the $k^{\text {th }}$ multicast group are assigned to the set index of $G_{k}$, and each receiver can only belong to a single multicasting group as depicted in Fig.3.1.

Each base station at time $t$ transmits a signal $\mathbf{x}_{b}(t)=\sum_{i=1}^{K} s_{i}(t) \mathbf{w}_{i b} \in \mathbb{C}^{N \times 1}$, where $s_{i}(t) \in \mathbb{C}^{N \times 1}$ is the $i^{t h}$ user data which is available at all base stations to be send synchronously and $\mathbf{w}_{i b} \in \mathbb{C}^{N \times 1}$ is the $i^{t h}$ beamform vector at base station $b$.

The received signal at the $m^{\text {th }}$ user at time $t$ is

$$
\mathbf{y}(t)=\mathbf{h}_{m}\left[\mathbf{x}^{H}{ }_{1}(t), \ldots, \mathbf{x}_{B}^{H}(t)\right]^{H}+\mathbf{n}_{m}
$$

were $\mathbf{h}_{m} \in \mathbb{C}^{1 \times N B}$ is the channel vector between all base stations, and the $m^{\text {th }}$ receiver and $\mathbf{n}_{m}$ is the zero-mean, circularly symmetric white Gaussian noise at the $m^{\text {th }}$ user.

Define $\mathbf{w}_{i}^{H}=\left[\mathbf{w}_{i 1}^{H}, \ldots, \mathbf{w}_{i B}^{H}\right] \in \mathbb{C}^{1 \times N B} \forall i \in[1,, K]$ as the aggregate beamform 


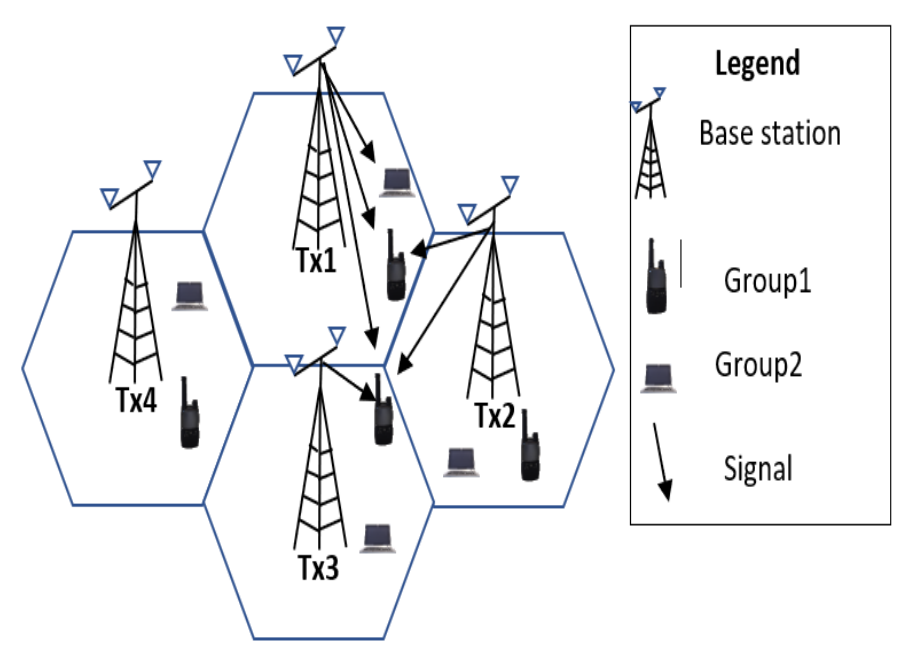

Figure 3.1: Multicast, mulicell, multigroup System model

vectors at the $m^{t h}$ user, and let $\mathbf{R}_{m} \in \mathbb{C}^{N B \times N B}$ denote the covariance channel matrix at the $m^{\text {th }}$ user which is $\mathbf{R}_{m}=\mathbf{h}_{m}^{H} \mathbf{h}^{m}$ for a slow fading channel and $\mathbf{E}\left[\mathbf{h}_{m}^{H} \mathbf{h}_{m}\right]$ for a fast fading channel and, let $\sigma_{m}^{2}$ denote the noise power. We can define the $S I N R_{m}$ at the $m^{\text {th }}$ user as:

$$
\operatorname{SIN} R_{m}\left(\left\{\mathbf{w}_{k}\right\}_{k=1}^{K}\right)=\frac{\mathbf{w}_{k}^{H} \mathbf{R}_{m} \mathbf{w}_{k}}{\sum_{i=1, i \neq k}^{K} \mathbf{w}_{i}^{H} \mathbf{R}_{m} \mathbf{w}_{i}+\sigma_{m}^{2}}
$$

Define a matrix:

$$
\mathbf{A}_{b}=\underset{\forall b \in(1, \ldots, B)}{\operatorname{diag}\left\{\mathbf{e}_{\mathbf{b}}\right\}} \otimes \mathbf{I}_{N}
$$

where $\mathbf{I}_{N}$ is $N \times N$ identity matrix. The problem of maximizing the minimum SINR over all users can be written as:

(P1) : 


$$
\begin{gathered}
\max _{\{\mathbf{w}\}_{k=1}^{K} .} \min _{k \in\{1 \ldots . . K\}} \min _{m \in G_{k}} \frac{\mathbf{w}_{k}^{H} \mathbf{R}_{m} \mathbf{w}_{k}}{\sum_{i=1, i \neq k}^{K} \mathbf{w}_{i}^{H} \mathbf{R}_{m} \mathbf{w}_{i}+\sigma_{m}^{2}} \\
\text { s.t. } \sum_{i=1}^{K}\left\|\mathbf{A}_{b} \mathbf{w}_{i}\right\|^{2} \leq P_{b}, \\
\forall b \in(1, \ldots, B)
\end{gathered}
$$

where $P_{b}$ is the maximum allowable transmitted power at base station $b$.

\subsubsection{Problem formulation}

In practice, the channels covariance matrix is unknown perfectly at the transmitter. Channel uncertainty has an important effect on system performance. Acquiring the exact channels can lead to better utilization of multiple input single output(MISO) and/or MIMO benefits by combating the system degradation where the highest SINR can be guaranteed at the receiver.

When the channel is subject to error, then the actual channel vector can be defined deterministically by

$$
\mathbf{h}_{m}=\widehat{\mathbf{h}}_{m}+\delta_{m}
$$

where $\mathbf{h}_{m}$ is the true channel vector, $\widehat{\mathbf{h}}_{m}$ is the estimated channel vector and $\delta_{\mathbf{m}}$ is the unknown error. This error can be described using different uncertainty sets but the most commonly used uncertainty set is the matrix norm [81]. In this work we will consider the Frobenius norm where the error is bounded by some predefined value. In particular, we write 


$$
\begin{gathered}
\mathbf{R}_{m}=E\left[\mathbf{h}_{\mathbf{m}} \mathbf{h}_{\mathbf{m}}^{\mathbf{H}}\right]=\widehat{\mathbf{R}}_{\mathbf{m}}+\Delta \mathbf{R}_{\mathbf{m}}(3.6 \mathrm{a}) \\
\left\|\Delta_{m}\right\|_{F} \leq \epsilon_{m} \\
\epsilon_{m}=S 1\left\|\widehat{\mathbf{R}}_{m}\right\|_{F} \\
\forall m \in(1, \ldots, M)
\end{gathered}
$$

where $0 \leq S 1 \leq 1$, is a constant that represents the normalized uncertainty. The relationship between $\delta_{m}$ and $\boldsymbol{\Delta} \mathbf{R}_{m}$ can be easily determined using

$$
\delta_{m}^{2}+2 \delta_{m}\left\|\widehat{\mathbf{h}}_{m}\right\|_{F}-\Delta \mathbf{R}_{m}=0
$$

Now using (3.6) and (3.7) the deterministic error in the channel matrix can be easily modeled.

Therefore, due to the channel uncertainty, the problem P1 becomes

$$
\begin{gathered}
\max _{\substack{\{\mathbf{w}\}_{k=1}^{K} . \\
\{\mathbf{R}\}_{m=1}^{M}}} \min _{k \in\{1, \ldots, K\}} \min _{m \in G_{k}} \frac{(P 2):}{\sum_{i=1, i \neq k}^{K} \mathbf{w}_{i}^{H}\left(\widehat{\mathbf{R}}_{m}+\Delta \mathbf{R}_{m}\right) \mathbf{w}_{i}+\sigma_{m}^{2}} \\
\text { s.t. } \widehat{\mathbf{R}}_{m}+\Delta \mathbf{R}_{m} \succeq 0 \\
\left\|\Delta \mathbf{R}_{m}\right\|_{F} \leq \epsilon_{m} \\
\sum_{k=1}^{K}\left\|\mathbf{A}_{b} \mathbf{w}_{k}\right\|^{2} \leq \mathbf{P}_{b},
\end{gathered}
$$

The objective function (3.8a) in problem P2 is not convex and fractional and dif- 
ficult to analyze. Following the procedure in $[85,88]$ we can replace the "max min" objective in problem P2 with a single-level maximization objective, by introducing an auxiliary variable $\gamma \geq 0$ and introducing new constraint (9b), allowing us to represent problem P2 with the following equivalent representation:

$$
\begin{gathered}
(P 3): \\
\max _{\{\mathbf{w}\}_{k=1}^{K},\{\boldsymbol{\Delta} \mathbf{R}\}_{m=1}^{M}, \gamma} \gamma \\
\text { s.t. } \frac{\mathbf{w}_{k}^{H}\left(\widehat{\mathbf{R}}_{m}+\Delta \mathbf{R}_{m}\right) \mathbf{w}_{k}}{\sum_{i=1, i \neq k}^{K} \mathbf{w}_{i}^{H}\left(\widehat{\mathbf{R}}_{m}+\Delta \mathbf{R}_{m}\right) \mathbf{w}_{i}+\sigma_{m}^{2}} \geq \gamma \\
\widehat{\mathbf{R}}_{m}+\Delta_{m} \succeq 0 \\
\left\|\mathbf{R}_{m}\right\|_{F} \leq \epsilon_{m} \\
\forall m \in G_{k}, \forall k \in\{1, \ldots, K\} \\
\sum_{k=1}^{K}\left\|\mathbf{A}_{b} \mathbf{w}_{k}\right\|^{2} \leq P_{b} \\
\forall b \in(1, \ldots, B)
\end{gathered}
$$

The constraint(3.9b) in problem P3 is still non convex and can be solved using different methods such as the SDR method [84], the SCA [85], and feasible point pursuit (FPP) method [97]. Here we will use the SCA method because we can find the beamforming vectors $\{\mathbf{w}\}_{k=1}^{K}$ directly without using semidefinte relaxation and the additional reformulation step.

In the literature there are three methods to resolve the channel uncertainty in downlink beamforming, these methods depend on the information available about 
the uncertainty matrices at the transmitter. The first method assumes that the uncertainty matrices is completely known at the transmitter and just inserts its values into the beamforming problem. The first solution does not have any practical value in spite of the fact that its has the best performance and least complexity as compared with other two solutions beacause it is difficult to acquire the uncertainty matrices at the transmitter as maintain previously. In subsequent sections we shall explain the other two practical solutions.

\subsection{Suboptimal solution}

This solution assumes that the uncertainty matrices is not known at the transmitter: neither its values nor the mathematical set to which it is belongs. The solution has the worst performance as compared with other two solution but less complixity as compard with the third solution that described in next section. This solution solves for the worst case following the solution in $[78,86,87,92]$. Here we will obtain the suboptimal solution using the SCA method as follows:

The first constraint in problem P3 (3.9b) consists of multiple optimization variables $\left(\{\mathbf{w}\}_{k=1}^{K},\{\boldsymbol{\Delta} \mathbf{R}\}_{m=1}^{M}, \gamma\right)$ so that problem P3 can not be solved as a single optimization problem. There are different approaches to solve this problem, and herein we will illustrate the conservative approach. More precisely, for a conservative solution, we obtain a worst case solution for the non-convex term (3.9b) with respect to $\{\Delta \mathbf{R}\}_{m=1}^{M}$ by minimizing the term (replacing it by its lower bound with respect to

$\left.\{\boldsymbol{\Delta} \mathbf{R}\}_{m=1}^{M}\right)$. This can be accomplished by replacing the numerator by its minimum 
and the denominator by its maximum, that is,

$$
\min _{\{\boldsymbol{\Delta} \mathbf{R}\}_{m=1}^{M}} S I N R_{m}=\frac{\min _{\{\mathbf{\Delta}\}_{m=1}^{M}} \mathbf{w}_{k}^{H}\left(\widehat{\mathbf{R}}_{m}+\mathbf{\Delta} \mathbf{R}_{m}\right) \mathbf{w}_{k}}{\max _{\{\mathbf{\Delta}\}_{m=1}^{M}} \sum_{i=1, i \neq k}^{K} \mathbf{w}_{i}^{H}\left(\widehat{\mathbf{R}}_{m}+\mathbf{\Delta} \mathbf{R}_{m}\right) \mathbf{w}_{i}}
$$

The worst case solution for the numerator is

$$
\min _{\{\boldsymbol{\Delta} \mathbf{R}\}_{m=1}^{M}} \mathbf{w}_{k}^{H}\left(\widehat{\mathbf{R}}_{m}+\underset{\left.\forall \mathbf{R}_{m}\right) \mathbf{w}_{k}=\mathbf{w}_{k}^{H}\left(\widehat{\mathbf{R}}_{m}-\epsilon_{m} I\right)^{+} \mathbf{w}_{k}}{\forall m \in(1, \ldots, M)}\right.
$$

Owing to different values of $\mathbf{R}_{m}$ and $\epsilon_{m} I$, the term $\left(\mathbf{R}_{m}-\epsilon_{m} I\right)$ is not positive semidefinite (Lemma 8.4.1, [98]). This term must be converted to a PSD matrix to ensure the existence and convergence of the solution (see section 8.2 for more details).

The worst case solution of the denominator is given by

$$
\begin{aligned}
& \max _{\{\mathbf{\Delta}\}_{m=1}^{M}} \sum_{i=1, i}^{K} \mathbf{w}_{i}^{H}\left(\widehat{\mathbf{R}}_{m}+\Delta \mathbf{R}_{m}\right) \mathbf{w}_{i} \\
&= \sum_{i=1, i \neq k}^{K} \mathbf{w}_{i}^{H}\left(\widehat{\mathbf{R}}_{m}+\epsilon_{m} I\right) \mathbf{w}_{i} \\
& \forall m \in(1, \ldots, M)
\end{aligned}
$$

Since the term $\mathbf{R}_{m}+\epsilon_{m} I$ is always PSD (Proposition 8.1.2, [98]), substituting (3.11) and (3.12) in the $S I N R_{m}$ constraint in problem P3, the problem is reduced and can be solved using SCA method [21] and explained explicitly in subsequent section as convex quadratically constrained quadratic programs (QCQP). This Convex QCQPs is solved using an interior point methods with complexity of $\mathcal{O}\left((M+1)^{1 / 2}(M+K N+2)(K N+1)^{2}\right)[85]$. As compared with the SDR method that 
solved using a bisection search method with complexity of $\mathcal{O}\left(K^{3} N^{6}+M K N^{2}\right)$ [85].

\subsection{Proposed(Robust) solution}

in the previous section we describe the suboptimal solution. In this section we will use the SCA method to find the robust solution which has better performance than the the suboptimal solution but with increased complexity. This solution assumes that the uncertainty matrices is unknown but the mathematical set to which its belong is known. Returning to problem P3 to illustrate the solution, we have to reformulate the problem as follows:

$$
\begin{gathered}
(P 4): \\
\text { s.t. } \gamma\left(\sum_{i=1, i \neq k}^{K} \operatorname{max.}_{\{\mathbf{w}\}_{k=1}^{K},\{\mathbf{\Delta}\}_{m=1}^{M}} \mathbf{w}_{i}^{H}\left(\widehat{\mathbf{R}}_{m}+\Delta \mathbf{R}_{m}\right) \mathbf{w}_{i}+\sigma_{m}^{2}\right) \\
-\mathbf{w}_{k}^{H}\left(\widehat{\mathbf{R}}_{m}+\Delta \mathbf{R}_{m}\right) \mathbf{w}_{k} \leq 0 \\
\mathbf{R}_{m}+\Delta \mathbf{R}_{m} \succeq 0 \\
\left\|\mathbf{R}_{m}\right\|_{F} \leq \epsilon_{m} \\
\forall m \in G_{k}, \forall k \in\{1, \ldots, K\} \\
\sum_{i=1}^{K}\left\|\mathbf{A}_{b} \mathbf{w}_{k}\right\|^{2} \leq \mathbf{P}_{b} \\
\forall b \in(1, \ldots, B)
\end{gathered}
$$

Problem (P4) consists of three optimization variables $\left(\gamma, \Delta \mathbf{R}_{m}\right.$, and $\left.\mathbf{w}_{i}\right)$, and hence 
cannot be solved as a single optimization problem. Here we shall handle P4 using an alternate-iterate technique. In particular, at each iteration we find a solution to a variable while holding the others fixed. The alternate-iterate technique will be illustrated in the following subsections.

\subsubsection{Solution for $\{\Delta \boldsymbol{R}\}_{m=1}^{M}$}

Let us define a matrix

$$
\mathbf{A}_{k}=\gamma \sum_{\substack{i=1, i \neq k \\ \forall k \in(1, \ldots, K)}}^{K} \mathbf{w}_{i}^{H} \mathbf{w}_{i}-\mathbf{w}_{k}^{H} \mathbf{w}_{k}
$$

and fix the variables $\gamma$ and $\mathbf{w}_{i}$. Then $\mathrm{P} 4$ reduces to the following optimization problem in $\Delta \mathbf{R}_{m}$

$$
\begin{gathered}
(P 5): \\
\max _{\{\mathbf{\Delta}\}_{m=1}^{M}} \operatorname{Tr}\left(\mathbf{A}_{i} \mathbf{\Delta} \mathbf{R}_{m}\right) \\
\text { s.t } \widehat{\mathbf{R}}_{m}+\Delta \mathbf{R}_{m} \succeq 0 \\
\left\|\Delta \mathbf{R}_{m}\right\|_{F} \leq \epsilon_{m} \\
\forall m \in G_{k}, \forall k \in\{1, \ldots, K\}
\end{gathered}
$$

P5 is a convex optimization problem in $\Delta \mathbf{R}_{m}$. We provide a next suboptimal closed form solution for $\Delta \mathbf{R}_{m}$. The proposed solution extends theorem 1 in [96] to solve (3.15a) where the author in [96] solve a problem similar to (3.15a) with $\mathbf{A}_{i}$ is positive(semi)definite here we extend the solution and solve the problem when the matrix $\mathbf{A}_{i}$ is Hermitian. Here $\boldsymbol{\Delta} \mathbf{R}_{m}$ can be found using the following equation 


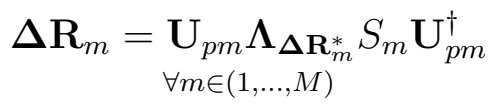

where $\Lambda_{\boldsymbol{\Delta} \mathbf{R}_{m}^{*}}$ is the diagonal eigenvalue matrix of $\boldsymbol{\Delta} \mathbf{R}_{m}^{*}$ that can be determined by:

$$
\lambda_{1}\left(\boldsymbol{\Delta} \mathbf{R}_{m}^{*}\right)=\epsilon_{m}\left[1+\sum_{\substack{n=1 \\ \forall m \in(1, \ldots, M)}}^{N} \frac{\left|\lambda_{n}\left(\mathbf{Y}_{m}\right)\right|}{\left|\lambda_{1}\left(\mathbf{Y}_{m}\right)\right|}\right]^{-.5}
$$

and

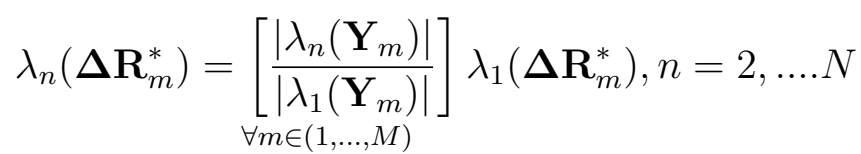

where $\lambda_{n}\left(\Delta \mathbf{R}_{m}^{*}\right)$ is the $n^{\text {th }}$ eigenvalue of the matrix $\Delta \mathbf{R}_{m}^{*}$, and $\lambda_{n}\left(\mathbf{Y}_{m}\right)$ is the $n^{t h}$ eigenvalue of the matrix $\mathbf{Y}_{m}$ (sorted in descending order) that can be determined by the eigendecomposition of $\mathbf{Y}\left(\mathbf{Y}_{m}=\mathbf{U}_{m} \boldsymbol{\Lambda}_{m} \mathbf{U}_{m}^{\dagger}\right)$, and $\mathbf{U}_{p m}$ can be found using

$$
\mathbf{U}_{p m}=\underset{\mathbf{U}_{m}}{ } \mathbf{P}_{m}
$$

where $\mathbf{P}_{m}$ is the permutation matrix whose location of entry is corresponding to the index of the sorted eigenvalue $\lambda_{n}\left(\mathbf{Y}_{m}\right)$. The matrix $\mathbf{Y}_{m}$ is found using:

$$
\underset{\forall m \in G_{k} \forall k \in\{1, \ldots, K\}}{\mathbf{Y}_{m}}
$$

where $\mathbf{Z}_{m}$ in (3.20) can be found using the following optimization problem:

$(P 6)$ : 


$$
\begin{aligned}
& \min _{\{\mathbf{Z}\}_{m=1}^{M} .} \epsilon_{m}\left\|\mathbf{A}_{i}+\mathbf{Z}_{m}\right\|_{F}+\operatorname{Tr}\left(\widehat{\mathbf{R}}_{m} \mathbf{Z}_{m}\right) \\
& \text { s.t. } \quad \mathbf{Z}_{m} \succeq 0
\end{aligned}
$$

Section 8.1 provides the proof for (3.17), (3.18), and (3.21). Recall that $\mathbf{A}_{i}$ can be determined using (3.14). Now note that problem P6 is convex and can be solved using NMSPG with linear computational complexity or a convex optimization package such as CVX.

\subsubsection{Solution for $\{\mathbf{w}\}_{k=1}^{K}$}

Substituting the obtained solution for $\Delta \mathbf{R}_{m}$ into P4 and removing the constraint of $\Delta \mathbf{R}_{m}$ we obtain:

$$
\begin{gathered}
(P 7): \\
\max _{\{\mathbf{w}\}_{k=1}^{K} .} \gamma \\
\text { s.t. } \gamma\left(\sum_{i=1, i \neq k}^{K} \mathbf{w}_{i}^{H}\left(\widehat{\mathbf{R}}_{m}+\Delta \mathbf{R}_{m}\right) \mathbf{w}_{i}+\sigma_{m}^{2}\right) \\
-\mathbf{w}_{k}^{H}\left(\widehat{\mathbf{R}}_{m}+\mathbf{\Delta R}_{m}\right) \mathbf{w}_{k} \leq 0 \\
\sum_{k=1}^{K} \| G_{k}, \forall k \in\{1, \ldots, K\} \\
\forall b \in(1,, \ldots, B) \\
\mathbf{A}_{b} \mathbf{w}_{k} \|^{2} \leq \mathbf{P}_{b}
\end{gathered}
$$

Problem P7 is not convex due to the non-convexity of the constraint $(3.22 \mathrm{~b})$, which contains two optimization variables. Replacing $\gamma^{t+1}$ with $\gamma^{t}+\Delta \gamma$ and $\mathbf{w}^{t+1}$ with $\mathbf{w}^{t}+$ $\Delta \mathbf{w}$ We can determine the beamforming vector using the SCA method. That is P7 
reduces to:

$$
\begin{gathered}
(P 8): \\
\max _{\left\{\mathbf{w}_{k=1}^{K} .\right.} \Delta \gamma \\
\text { s.t. }-\left(\mathbf{w}_{k}^{(t) H}\left(\widehat{\mathbf{R}}_{m}+\Delta \mathbf{R}_{m}\right) \mathbf{w}_{k}^{(t)}+2 \Re\left(\Delta \mathbf{W}_{k}^{H}\left(\widehat{\mathbf{R}}_{m}+\Delta \mathbf{R}_{m}\right) \mathbf{w}_{k}^{(t)}\right)\right. \\
+\gamma\left(\sum_{i=1, i \neq k}^{K}\left(\mathbf{w}_{k}^{(t) H}+\Delta \mathbf{w}\right)\left(\widehat{\mathbf{R}}_{m}+\Delta \mathbf{R}_{m}\right)\left(\mathbf{w}_{k}^{(t)}+\Delta \mathbf{w}\right)\right) \\
+\Delta \gamma\left(\sum_{i=1, i \neq k}^{K} \mathbf{w}_{k}^{(t) H}\left(\widehat{\mathbf{R}}_{m}+\Delta \mathbf{R}_{m}\right) \mathbf{w}_{k}^{(t)}\right) \\
+\sigma_{m}^{2}(\Delta \gamma+\gamma) \leq 0 \\
\forall m \in G_{k}, \forall k \in\{1, \ldots, K\} \\
\sum_{i=1}^{K}\left\|\mathbf{A}_{b}\left(\Delta \mathbf{w}_{i}+\mathbf{w}\right)_{k}\right\|^{2} \leq \mathbf{P}_{b} \\
\forall b \in(1, \ldots, B)
\end{gathered}
$$

We iteratively alternate between solutions for $\Delta \mathbf{R}_{m}$ in 3.5.1 and for $\Delta \mathbf{w}_{i}$ and $\Delta \gamma$ in 3.5.2 in order to solve the optimization problems, so that the beamforming vector is extracted. The iteration is stopped when the precision (which is the difference between $\gamma^{t+1}$ and $\gamma^{t}$ ) becomes less than or equal to a predefined value.

Conditions $\widehat{\mathbf{R}}_{m}+\boldsymbol{\Delta} \mathbf{R}_{m} \succeq 0$ and $\Delta \gamma>0$ (see section 8.2) ensure the existence and convergence of the solution.

The proposed robust solution involves iterating between solving problem P6 presented in section 3.5.1, which can be solved using NMSPC with linear computational 
complexity, $3 N+\mathcal{O}(1)$, and solving problem $\mathrm{P} 8$ presented in section 3.5.2, which can be solved using an interior point method with the SCA method having complexity $\mathcal{O}\left((M+1)^{1 / 2}(M+K N+2)(K N+1)^{2}\right)$ [85]. thus the total complexity is $\left.\left.\left(3 N+\mathcal{O}(1)+\mathcal{O}\left((M+1)^{(} 1 / 2\right)(M+K N+2)\left(K N+1^{2}\right)\right)\right)\right)$ per iteration which still require less complexity as compared with the QOS solution that uses bisection search method ( whose complexity is presented in the previous section)

For convergence analysis please refer to section 5.8.2(appendix 2) . Algorithm 1 presents this proposed robust solution.

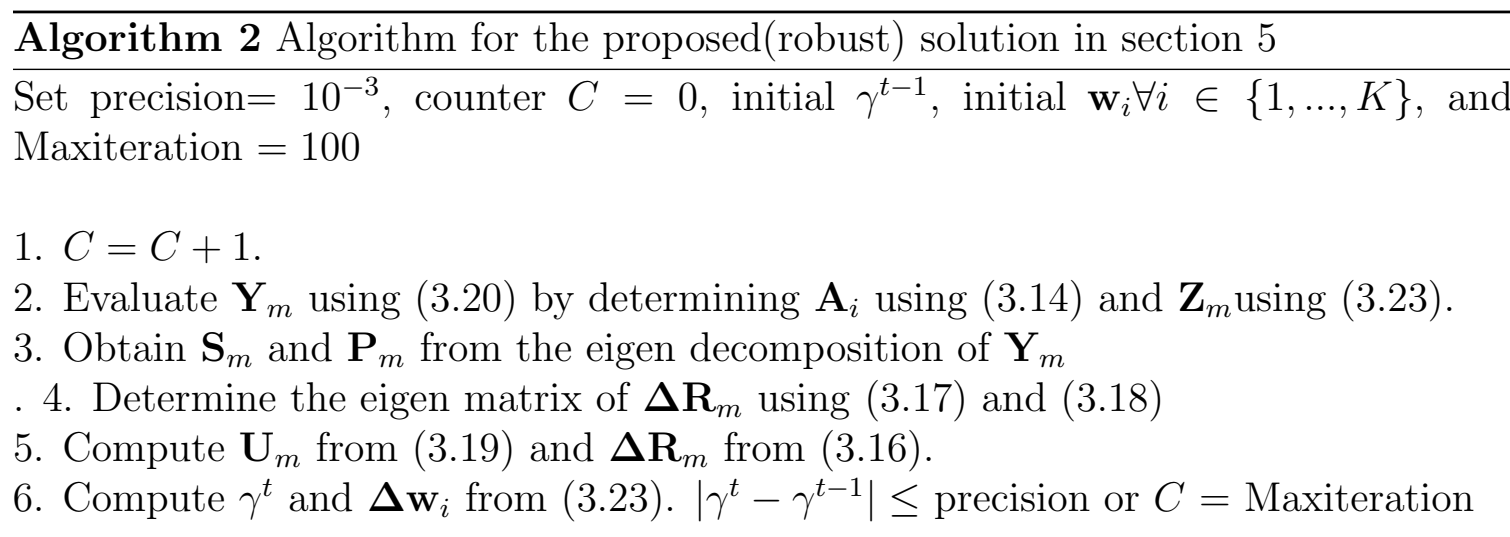

\subsection{Numerical Results}

We demonstrate our methodology using simulated data, and illustrate the convergence and existence of our solution over different parameter settings. The initial beamform vectors $\mathbf{w}_{i} \in \mathbb{C}^{N \times 1} \forall i \in[1, K]$ are randomly generated from a complex zero-mean circular Gaussian distribution with unit variance and the estimated covariance CSI is assumed for slow fading channels where $\widehat{\mathbf{R}}_{m}=\widehat{\mathbf{h}}_{m} \widehat{\mathbf{h}}_{m}^{H} \in \mathbb{C}^{N B \times N B}$ and 
$\widehat{\mathbf{h}}_{m} \in \mathbb{C}^{N B \times 1} \forall m \in[1,, M]$ is randomly generated from a complex zero-mean circular Gaussian distribution with unit variance. Throughout our simulation study we use a precision equal to $10^{-3}$ and demonstrate the results for three solution methods for imperfect CST

1)the robust solution.

2)the suboptimal solution.

3)the non-robust solution.

In each simulation study an average is taken based on 500 Monte-Carlo runs.

For the first example in Fig.2, we set $N=5, M=12, k=2$ and3, $\sigma_{m}=1 \forall m \in$ $[1,, M], B=1 \forall b \in[1, B], S=.1$ and $P_{b}=10^{2}$. The figure presents the convergence of the three cases in about the first ten iterations. Also, the figure shows that our proposed solution is more power efficient than the sub-optimal solution proposed in $[86,87]$, while the non-robust solution has the best power performance among the three solutions. This is due to the fact that the non-robust solution maximizes the minimum SINR among all receivers without considering the uncertainty in the covariance CSI, while the robust solution maximizes the minimum SINR among all receivers and across all uncertainty. Moreover, the results show that as the number of groups increases the minimum SINR decreases due to the fact that as the number of group increases the interference signal at each user increases leading to a decrease of SINR achieved at the user, see equation (3.2).

For the second example in Fig.3, we set $N=8$ and $12, M=4, L=2, \sigma_{m}=1 \forall m \in$ 


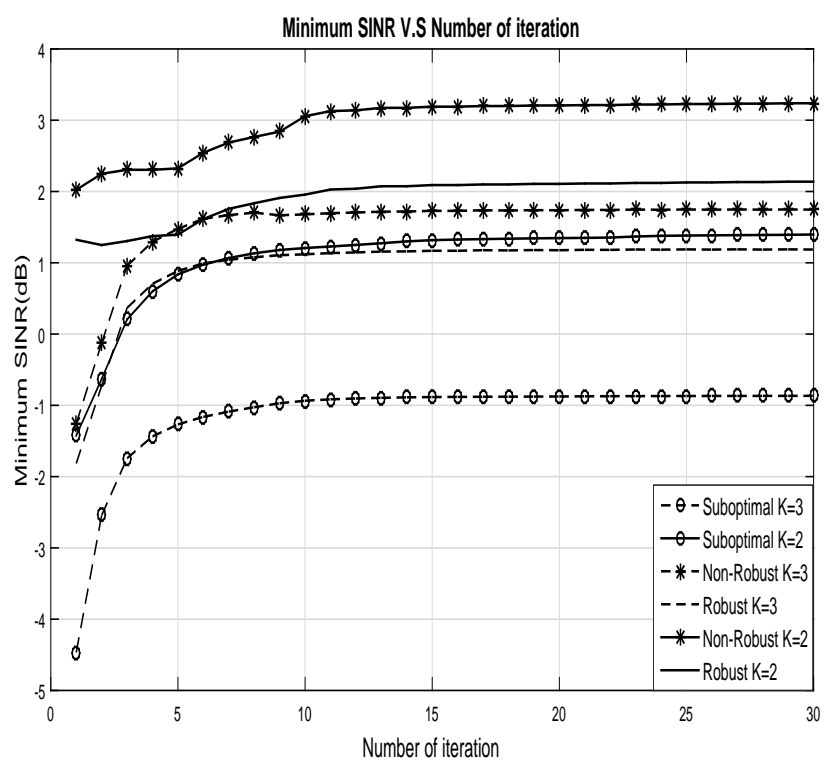

Figure 3.2: Minimum SINR versus the number of iterations for $\mathrm{k}=2$ and 3 group

$[1,, M], B=1 \forall b \in[1,, B]$, and $S=.1$. We compare the minimum SINR with the minimum transmitted power for the tow cases with the robust solution that uses the QOS beamforming technique [94] for the tow transmit antenna value. We find that our robust solution has better power performance when compared to the QOS method and moreover, we see that as the number of transmit antennas increases, the received power also increases and hence the minimum SINR increases. This is due to the fact that as the number of transmit antennas increases the degree of freedom in beamforming increases too and hence more concentrated power is received at the receivers which leads to high SINR.

The third example is presented in Fig.4. Here $N=5, M=12, L=2, \sigma_{m}=1 \forall m \in$ $[1,, M], B=1 \forall b \in[1, B]$ and $P_{b}=10^{-2}$. These results examine the impact of the value of the normalized uncertainty on the received minimum SINR and we notice 


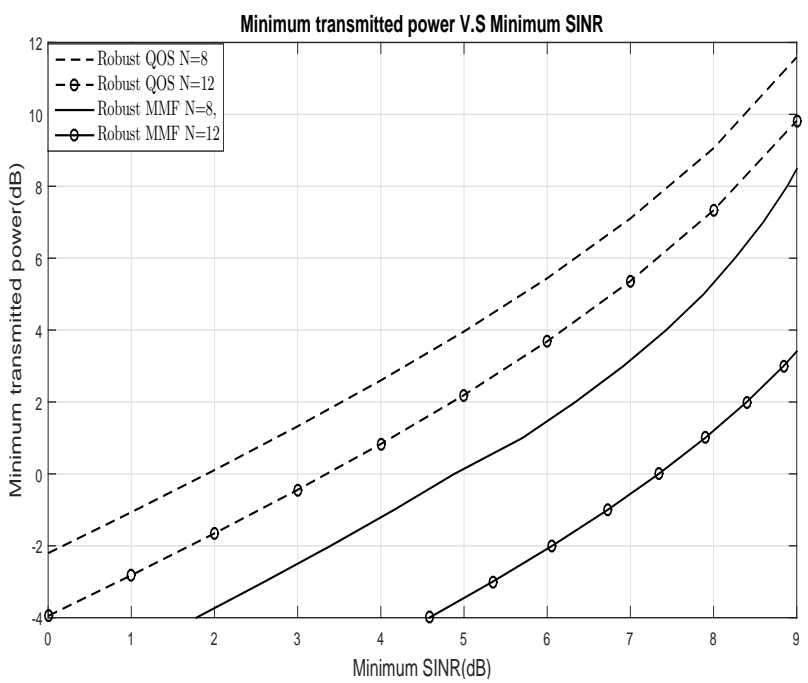

Figure 3.3: Minimum transmitted power versus minimum received SINR for different numbers of transmit antenna and different robust solutions

that as the normalized uncertainty increases the received minimum SINR decreases.

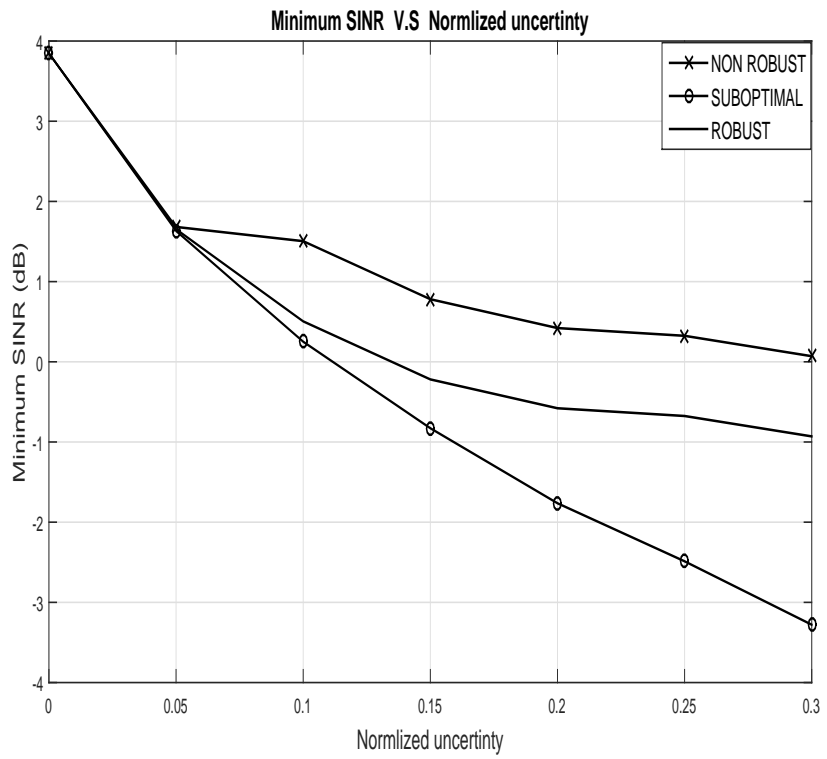

Figure 3.4: Minimum SINR versus normalized uncertainty

Fig.5, presents a fourth example with $N=6, S 1=.1, L=2, \sigma_{m}=1 \forall m \in$ 
$[1,, M], B=1 \forall b \in[1,, B]$ and $P_{b}=10^{-2}$. These results illustrate the minimum SINR versus the number of users per group. Here, the minimum SINR decreases as the number of users per group increases due to the fact that as the number of users increases, the interference at each user increases as well, leading to decreased to SINR at the receivers.

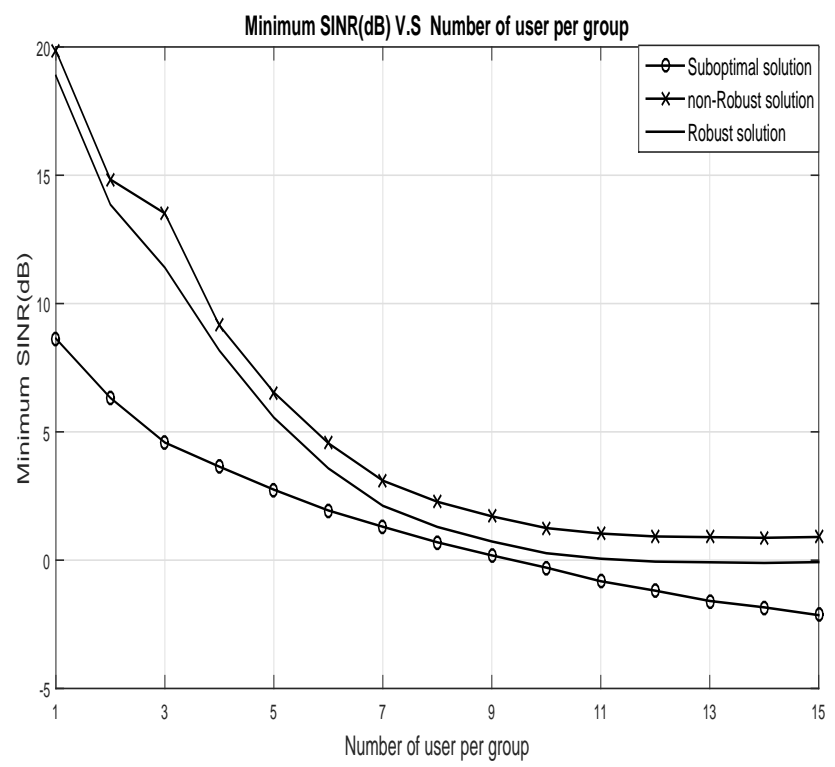

Figure 3.5: Minimum SINR versus the number of users per group for three solutions

Finally Table1 shows the effect of the number of base stations on the received minimum SINR. From the table we observe that as the number of base stations increases the received power at each receiver increases as well, and hence the received minimum SINR increases.

Fig. 3 indicates that the robust solution presented here requires less transmitted power to achieve the same SINR at the receiver as compared with the QOS method. 
Table 3.1: Minimum SINR versus number of base station for three cases

\begin{tabular}{|c|c|c|c|}
\hline \multirow{2}{*}{ Number of base station } & \multicolumn{3}{|c|}{ Minimum SINR(dB) } \\
\cline { 2 - 4 } & Robust & non-Robust & sub-optimal \\
\hline 1 & -1.405 & -.958 & -2.075 \\
\hline 2 & 2.702 & 2.98 & -.417 \\
\hline 3 & 6.96 & 8.01 & .052 \\
\hline
\end{tabular}

Also, figure 2,4,5 and table -1- shows that our method has better SINR as compared with the suboptimal solution that follows $[86,87]$. This suggests that the robust solution presented here has improved power efficiency with the existence solutions of multiuser beamforming with non-perfect CSI

\subsection{Conclusion}

In this chapter, we present a novel technique(robust solution) for optimizing the design of downlink beamforming vectors for a multicast, multigroup, multicell system. Because the CSI is subject to uncertainty, we utilize a robust optimization procedure in an SCA approach, avoiding the approximation step necessary in the SDR. We presented an extensive computational simulation, demonstrating the superior performance of our approach when compared to a non-robust solution that does not account for uncertainty, and a sub-optimal approach proposed in $[86,87]$. For future work, we will consider our approach to solve the uncertainty problem in cognitive radio networks if the CSI of secondary users and the interference CSI from secondary transmitters to primary receivers is subject to uncertainty. This is subject of great interest in practical applications.. 


\subsection{Appendices}

\subsubsection{Appendix 1: proof for(3.17),(3.18) and (3.23)}

Consider the optimization problem

$$
\begin{gathered}
(P 5): \\
\max _{\{\boldsymbol{\Delta} \mathbf{R}\}_{m=1}^{M}} \operatorname{Tr}\left(\mathbf{A}_{i}\left(\boldsymbol{\Delta} \mathbf{R}_{m}\right)\right) \\
\text { s.t. } \widehat{\mathbf{R}}_{m}+\Delta \mathbf{R}_{m} \succeq 0 \\
\left\|\mathbf{\Delta R}_{m}\right\|_{F} \leq \epsilon_{m} \\
\forall m \in G_{k}, \forall k \in\{1, \ldots, K\}
\end{gathered}
$$

and use the general definition of the Frobenius norm of $x \in \mathbb{C}^{n \times m}$ :

$$
\|\mathbf{X}\|_{F}=\left(\sum_{i=1,}^{\min (n, m)}\left(\lambda_{i}(\mathbf{X})\right)^{2}\right)^{1 / 2}
$$

Define $\mathbf{Z}_{m}$ and $\mu_{m}$ as a Lagrange multiplier so that the dual function associated with (3.24) is:

$$
\begin{gathered}
f\left(\Delta \mathbf{R}_{m}, \mathbf{Z}_{m}, \mu_{m}\right)=\operatorname{Tr}\left(\boldsymbol{\Delta} \mathbf{R}_{m} \mathbf{A}_{i}\right)+\operatorname{Tr}\left(\mathbf{Z}_{m}\left(\widehat{\mathbf{R}}_{m}+\Delta_{m}\right)\right) \\
-\mu_{m}\left(\left\|\mathbf{\Delta R}_{m}\right\|_{F}-\epsilon_{m}\right) \\
f\left(\Delta \in G_{k}, \forall k \in\{1, \ldots, K\}\right. \\
\left.\mathbf{\Delta R}_{m}, \mathbf{Z}_{m}, \mu_{m}\right)=\operatorname{Tr}\left(\Delta \mathbf{R}_{m}\left(\mathbf{Z}_{m}+\mathbf{A}_{i}\right)\right)-\mu_{m}\left(\left\|\Delta \mathbf{R}_{m}\right\|_{F}-\epsilon_{m}\right)
\end{gathered}
$$




$$
\underset{\forall m \in G_{k}, \forall k \in\{1, \ldots, K\}}{+\operatorname{Tr}\left(\mathbf{Z}_{m} \widehat{\mathbf{R}}_{m}\right)}
$$

Using the fact 5.12.4 from $[33]\left(\operatorname{Tr}(\mathbf{X Y}) \leq \sum_{i=1}^{n} \lambda_{i}(\mathbf{X}) \lambda_{i}(\mathbf{Y})\right.$, for $\mathbf{X}$ and $\mathbf{Y} \in \mathbb{C}^{n \times n}$ and the definition of the norm from (3.25), then (3.27) reduces to

$$
\begin{gathered}
f\left(\boldsymbol{\Delta} \mathbf{R}_{m}, \mathbf{Z}_{m}, \mu_{m}\right)=\operatorname{Tr}\left(\boldsymbol{\Lambda}_{\mathbf{Y}_{m}} \boldsymbol{\Lambda}_{\Delta \mathbf{R}_{m}}\right)+\mu_{m}\left(\left\|\boldsymbol{\Lambda}_{\boldsymbol{\Delta} \mathbf{R}_{m}}\right\|_{F}-\epsilon_{m}\right) \\
+\operatorname{Tr}\left(\mathbf{Z}_{m}\left(\widehat{\mathbf{R}}_{m}+\boldsymbol{\Delta} \mathbf{R}_{m}\right)\right)(3.28)
\end{gathered}
$$

where $\mathbf{Y}_{m}=\mathbf{Z}_{m}+\mathbf{A}_{i} \forall m \in G_{k} \forall k \in\{1, \ldots, K\}$ using corollary 9.3.9 [98] $\left(\operatorname{Tr}(\mathbf{X Y}) \leq\|\mathbf{X}\|_{F}\|\mathbf{Y}\|_{F}\right)$, (3.28) becomes:

$$
\begin{aligned}
\mathbf{f}\left(\boldsymbol{\Delta} \mathbf{R}_{m}, \mathbf{Z}_{m}, \mu_{m}\right) & \leq\left\|\boldsymbol{\Lambda}_{\mathbf{Y}_{m}}\right\|_{F}\left\|\boldsymbol{\Lambda}_{\Delta \mathbf{R}_{m}}\right\|_{F}+\mu_{m}\left(\left\|\boldsymbol{\Lambda}_{\Delta \mathbf{R}_{m}}\right\|_{F}-\epsilon_{m}\right) \\
& +\operatorname{Tr}\left(\mathbf{Z}_{m}\left(\widehat{\mathbf{R}}_{m}+\Delta_{m}\right)\right)(3.29)
\end{aligned}
$$

The inequality in (3.29) is reduced to equality if and only if

$$
\frac{\lambda_{1}\left(\Delta_{m}\right)}{\lambda_{1}\left(\mathbf{Y}_{m}\right)}=\ldots=\frac{\lambda_{n}\left(\Delta_{m}\right)}{\lambda_{n}\left(\mathbf{Y}_{m}\right)}
$$

(for more details see proposition 9.1.9 [98]). Using the definition of the Frobenius norm in (3.25) the upper bound value of $\left\|\boldsymbol{\Delta} \mathbf{R}_{m}\right\|_{F}$ then, $\epsilon_{m}$ can be found using:

$$
\epsilon_{m}^{2}=\lambda_{1}^{2}\left(\underset{\forall m \in(1, \ldots, M)}{\left.\Delta \mathbf{R}_{m}\right)+, \ldots,}+\lambda_{n}^{2}\left(\Delta \mathbf{R}_{m}\right)\right.
$$

Plugging (3.30) in (3.31) leads to (3.17). 
Equation (3.18) can be obtained from (3.17) and (3.30) to evaluate the value of $\mathbf{Z}_{m}$. Moreover, $\mu_{m}$ has two potential bound limits, either $\left\|\boldsymbol{\Lambda}_{\mathbf{Y}_{m}}\right\|_{F}>\mu_{m}$ or $\mu_{m} \geq\left\|\boldsymbol{\Lambda}_{\mathbf{Y}_{m}}\right\|_{F}$. Based on these two limits the upper limits of (3.29) are:

$$
f\left(\mathbf{Z}_{m}, \mu_{m}\right) \leq \begin{cases}\infty, & \mu_{m}<\left\|\boldsymbol{\Lambda}_{\mathbf{Y}_{m}}\right\|_{F} \\ \mu_{m} \epsilon_{m}+\operatorname{Tr}\left(\widehat{\mathbf{R}}_{m} \mathbf{Z}_{m}\right), & \mu_{m} \geq\left\|\boldsymbol{\Lambda}_{\mathbf{Y}_{m}}\right\|_{F}\end{cases}
$$

since $\mu_{m}$ must be as small as possible. Then we choose the value of $\mu_{m}=\left\|\boldsymbol{\Lambda}_{\mathbf{Y}_{m}}\right\|_{F}$ and this reduces (3.29) to:

$$
\begin{aligned}
& (P 6): \\
& \max _{\left\{\mathbf{Z}_{m}\right\}_{m=1}^{M}} \epsilon_{m}\left\|\mathbf{A}_{i}+\mathbf{Z}_{m}\right\|_{F}+\operatorname{Tr}\left(\widehat{\mathbf{R}}_{m} \mathbf{Z}_{m}\right) \\
& \text { s.t. } \quad \mathbf{Z}_{m} \succeq 0
\end{aligned}
$$

\subsubsection{Appendix 2: proof of feasibility and convergence of P8}

First, note that for any $\mathbf{x} \in \mathbb{C}^{n \times 1}$ and $\mathbf{A} \in \mathbb{C}^{n \times n}$ a PSD matrix we have

$$
\mathbf{x}^{H} \mathbf{A} \mathbf{x} \geq 0
$$

Case 1: Assume that $\mathbf{w}_{i} \in \mathbb{C}^{n \times 1}, \Delta \mathbf{w}_{i}=0$ and $\Delta \gamma=0$ in (3.23b). Then 


$$
\frac{\mathbf{w}_{k}^{H}\left(\widehat{\mathbf{R}}_{m}+\boldsymbol{\Delta} \mathbf{R}_{m}\right) \mathbf{w}_{k}}{\left.\sum_{i=1, i \neq k}^{K} \mathbf{w}_{i}^{H} \widehat{\mathbf{R}}_{m}\right)+\boldsymbol{\Delta}_{m} \mathbf{w}_{i}+\sigma_{m}^{2}} \geq \gamma
$$

Thus $\mathrm{P} 8$ reduces to $\mathrm{P} 2$, which means that $\Delta \mathbf{w}_{i}=0$ and $\Delta \gamma=0$ are feasible solutions.

Case $2: \mathbf{w}_{i} \in \mathbb{C}^{n \times 1}, \Delta \mathbf{w}_{i}=0$ and $\Delta \gamma \neq 0$

Substituting the above values in $(3.23 \mathrm{~b})$, the equation reduces it to:

$$
\frac{\left.\mathbf{w}_{k}^{H} \widehat{\mathbf{R}}_{m}+\Delta \mathbf{R}_{m}\right) \mathbf{w}_{k}}{\left.\sum_{i=1, i \neq k}^{K} \mathbf{w}_{i}^{H} \widehat{\mathbf{R}}_{m}+\Delta \mathbf{R}_{m}\right) \mathbf{w}_{i}+\sigma_{m}^{2}} \geq \gamma+\Delta \gamma
$$

By contradiction, if $\Delta \gamma$ is negative then (3.36) violates $\mathrm{P} 2$ and hence $\Delta \gamma$ must be positive. Thus, according to case1 and case2, then $\Delta \gamma$ must be greater then or equal to zero

Case3: Gonsider $\mathbf{w}_{i} \in \mathbb{C}^{n \times 1}, \Delta \mathbf{w}_{i} \in \mathbb{C}^{n \times 1}$ and $\Delta \gamma \geq 0$. The proof of this case is similar to that in [20] as follows.

Following the results from case 1 and case 2, and substituting the above values in (3.23b) we obtain the following:

$$
\begin{aligned}
& \left(\mathbf{w}_{k}^{(t) H}\left(\widehat{\mathbf{R}}_{m}+\Delta \mathbf{R}_{m}\right) \mathbf{w}_{k}^{(t)}+2 \Re\left(\Delta \mathbf{w}_{k}^{H}\left(\widehat{\mathbf{R}}_{m}+\Delta \mathbf{R}_{m}\right) \mathbf{w}_{k}^{(t)}\right)\right. \\
\geq & \gamma\left(\sum_{i=1, i \neq k}^{K}\left(\mathbf{w}_{k}^{(t) H}+\Delta \mathbf{w}\right)\left(\widehat{\mathbf{R}}_{m}+\Delta \mathbf{R}_{m}\right)\left(\mathbf{w}_{k}^{(t)}+\Delta \mathbf{w}\right)+\sigma^{2}\right)
\end{aligned}
$$




$$
+\Delta \gamma\left(\sum_{i=1, i \neq k}^{K} \mathbf{w}_{k}^{(t) H}\left(\widehat{\mathbf{R}}_{m}+\Delta \mathbf{R}_{m}\right) \mathbf{w}_{k}^{(t)}+\sigma_{m}^{2}\right)
$$

We add the term $\boldsymbol{\Delta} \mathbf{w}_{k}^{H}\left(\widehat{\mathbf{R}}_{m}+\Delta_{m}\right) \boldsymbol{\Delta} \mathbf{w}_{k} \geq 0$ to the left hand side of (3.37) (following definition (3.34)) this does not affect the inequality. Similarly, we can divide both sides by $\sum_{i=1, i \neq k}^{K}\left(\mathbf{w}_{k}^{(t) H}+\Delta \mathbf{w}\right)\left(\widehat{\mathbf{R}}_{m}+\Delta \mathbf{R}_{m}\right)\left(\mathbf{w}_{k}^{(t)}+\Delta \mathbf{w}\right)+\sigma^{2}$ and define

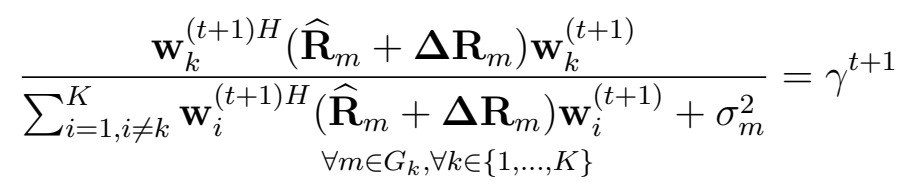

As a result, this reduces (3.37) to

$$
\begin{gathered}
\Delta \gamma \frac{\sum_{i=1, i \neq k}^{K} \mathbf{w}_{k}^{(t) H}\left(\mathbf{R}_{m}+\Delta \mathbf{R}_{m}\right) \mathbf{w}_{k}^{(t)}+\sigma_{m}^{2}}{\sum_{i=1, i \neq k}^{K}\left(\mathbf{w}_{k}^{(t) H}+\Delta \mathbf{w}\right)\left(\widehat{\mathbf{R}}_{m}+\Delta \mathbf{R}_{m}\right)\left(\mathbf{w}_{k}^{(t)}+\Delta \mathbf{w}\right)+\sigma^{2}} \\
+\gamma^{t} \leq \gamma^{t+1} \\
\forall m \in G_{k}, \forall k \in\{1, \ldots, K\}
\end{gathered}
$$

The ratio on the right hand side of the constraint above is always positive following (3.34), thus the new iteration $\gamma^{t+1}>\gamma^{t}$, provided that $\Delta \gamma \neq 0$. Thus, Algorithm 1 must converge in a finite number of iterations. 


\section{Chapter 4}

\section{Robust Underlay Cognitive Network Download Beamforming In Multiple Users,Multiple Groups Multicell Scenario}

\subsection{Introduction}

Achieving high quality of service (QOS) at the end users while maintaining the low interference power at the primary users is the main goal in underlay cognitive radio (CR) networks. This goal becomes a more difficult task in the designing of beamforming vectors where all channels state information (CSI) in the secondary network as well as the interference CSIs are uncertain. This task is addressed in this chapter using iterative optimization technique. In this technique the original CSI problem, which is difficult to solve as a single optimization problem, is instead separated into two sub-problems. The first sub-problem is the interference power problem, which 
can be solved either sub-optimally or optimally using Lagrange duality. The second sub-problem is the QOS problem, which can be solved either sub-optimally or robustly using Non-monotone spectral projected gradient method (NMSPG). The two sub-problem solutions are then recombined into a single problem to extract beamforming vectors. Two methods are invoked to extract the beamforming vectors: either the successive convex approximation (SCA) method or the bisection search method. Theoretical analysis and simulation results indicate that the two methods can offer a tradeoff between better QOS (using bisection search method) or less computational complexity (using SCA method).

\subsection{Background}

A resource that is very precious in wireless communication is the radio spectrum [100] which is regulated by the Federal Communication Commission (FCC). The sever demands for high data rate due to multimedia applications [101] and the growth in new wireless technologies [102] has led to noticeable shortage in radio spectrum when using conventional wireless communication paradigms [101]. Most of the spectrum is underutilized because of the spectrum utilization depending on time and place of wireless terminal(s) as reported by the FCC [103]. Mitola and Maguire were the first who proposed cognitive radio network (CRN) in 1992 [104] which is a promising technique to solve the unfair radio spectrum utilization problem [105] through dynamic frequency allocation to the secondary users without interfering primary users [106-108]

There are two type of CRN. the first is overlay CRN in which the primary user(s) has the highest priority to utilize the spectrum and the secondary user can use the 
spectrum if it is not utilized by the primary user. The secondary user must have the ability to detect the spectrum hole(s) ( the frequencies that are not used by the primary user) through an available detection techniques such as energy detector [109], match filter detector [110], likelihood ratio test detector [111], cyclostationary detector [112], Bayesian based GLRT detector [113]...,etc. or 2). The other CRN type is underlay CRN in which the primary user and the secondary user share the spectrum resource simultaneously. Here both users must assure their QOS but the secondary user must not interfere with the primary user through the use of different approaches such as interference alignment $[114,115]$. In this chapter we will consider the underlay CRN paradigm.

Another promising technique that can improve spectrum use efficiency in wireless communication is the multiple input multiple output (MIMO) technique which can reduce the interference to the users. Integrating MIMO technique in CRN can significantly improve the performance of a wireless communication system and open a new gate of applications and research in wireless communication. The full benefit of MIMO in CRN can be exploited when the channel state information (CSI) are perfectly gathered, thus much research has been done in this area such as [72,116-118]. In practice and due to the nature of wireless communication channels, the estimated CSI is subject to error, and much research attention has been given to its mitigation in wireless communication $[75,82]$.

In down-link beamforming there are two types of solutions to mitigate the effect of CSI uncertainty: 1) the sub-optimal solution, in this solution the beamforming vec- 
tors are extracted under worst case conditions; or 2)robust beamforming, by which the effect of CSI uncertainty is mitigated according to a user-specified level of robustness. The authors in [119] find a suboptimal solution in CRN with uncertain CSI. The authors in [95] find the solution in CRN using both solution while the author in [94] find robust beamforming only in CRN. All of the solutions proposed by these authors use semidefinite relaxation(SDR).

In SDR the optimization problem is relaxed to eliminate the rank constraint and this relaxation is the main drawback of the SDR method since a reformulation step is required as an additional approximation step to extract beamforming vectors. Although, the authors in $[71,76]$ found solutions for the drank constraint and produced beamforming matrices of rank two and general rank respectively, the rank two solution requires an additional reformulation step and the general rank reduces the rate of the system. Also, the SDR method suffers from high computational complexity as compared with the SCA method. In previous work [120] we have designed the beamforming vector using the SCA method and extracted beamforming vectors directly for multicell multiple group conventional networks.

In this chapter we examine the design of the beamforming vectors in underlay $\mathrm{CR}$ networks. The networks assume multiple groups of multiple broadcast users. The network consists of multi-base stations at the transmitting side that utilize the frequency spectrum of multiple primary users. The estimated CSIs of the secondary network as well as the CSIs of the interference network are assumed to be non-perfect and subjected to error. Our solution uses both the SCA method and the bisection search method to avoid the additional reformulation step that lead to error in the 
solution.

Since the problem is difficult to be solve as a single optimization problem, for reasons that will be explained in subsequent sections, an iterative optimization technique is utilized to solve the problem and extract beamforming vectors, with the original problem divided into two sub-problems: the QOS problem and the interference power problem. Two types of solution are proposed for finding the beamforming vectors: the sub-optimal solution and the robust solution

The main contributions of the current chapter are as follows:

1) We extract the beamforming vectors in CRN with multicell multiple groups that achieve high QOS at the secondary users while maintaining the interference levels below small predefined values at the primary users, using the SCA method for suboptimal and robust cases.

2) We invoke the bisection search method to extract beamforming vectors for the robust case for the CR system proposed in 1 .

3)The sub-optimal solution is determined for the secondary CSI's uncertainty in the QOS problem as well as for interference CSI's uncertainty in the interference power problem.

4) In the QOS, the solution for the CSI uncertainty is extracted robustly while an optimal solution is found for the CSIs uncertainty in the interference power problem. The optimal solution is determined via a closed form solution using Lagrange duality Because our procedure obtains the optimal solution to the Lagrangian dual for a convex primal problem with a strictly feasible solution (Slater's constraint qualification) we obtain the optimal solution to the corresponding primal problem. 
5)We prove our deterministic solution for the interference sub-optimization problem is equivalent to the stochastic optimal solution.

\subsection{Chapter orgnization And Notation}

The chapter are organized as follows. Section 2 presents a complete description of our model for multicell, multigroup multicast for an underlay CR network system with beamforming formulation using a MIN-MAX beamforming technique in which non-perfect CSIs for both primary and secondary user channels are assumed. In section 3 sub-optimal beamforming vectors are extracted using the SCA method after mitigating the effect of channels uncertainty under worst-case conditions. Section 4 describes a robust solution to extract the beamforming vectors where two methods are invoked (the SCA and bisection search methods). Section 5 presents our simulation results, examining the impact of different system parameters on the feasibility and convergence of our solutions. Finally, section 6 presents our conclusions.

Throughout this chapter we use uppercase/lowercase boldface letters to denote the matrices/column vectors respectively, the lite-face letters to represent scalars, the notation $E[\mathbf{X}]$ and $\mathbf{X} \succeq 0$ to represent the statistical expectation of the random matrix $\mathbf{X}$ and a PSD matrix respectively, the operations $\|\mathbf{X}\|_{F}$ and $|\mathbf{X}|$ are the Frobenius norm and the absolute value of the matrix $\mathbf{X} \operatorname{diag}\left(\mathbf{e}_{b}\right)$ and $\mathbf{I}_{N}$ is $N \times N$ identity matrix, $\mathbb{C}^{m \times n}$ represent $m \times n$ complex matrix, the operation $(.)^{H}$ is the complex conjugate transpose of a matrix and/ or vector, the operation $(\mathbf{X})^{H}$ means that the definite matrix must be converted to PSD matrix, and finally the Kronecker product 
is represented by $\otimes$

\subsection{System model and problem statement}

Consider an underlay cognitive radio scenario in which B secondary user transmitters (SU-T) with $N$ transmit antennas utilize frequency resources of B primary users (PU) and perform MIMO transmission to serve synchronously $M$ single antenna secondary users receivers (SU-R). Assume the cognitive transceivers and the primary users receivers (PU-R) reside on the same network tire while the primary users transmitters (PU-T) reside on the other network tire to avoid interference caused by the (PU-T) on the (SU-R).

The SU-R users are assumed to be scheduled into $K$ multicast groups $\left\{G_{1}, \ldots, G_{K}\right\}$ (the users, grouping is beyond the scope of this chapter).Each receivers in the $k^{\text {th }}$ multicast group, thus is assigned to the set index of $G_{k}$, and each receiver can only belong to a single multicasting group $G_{i} \cap G_{j}=\emptyset$ where $i \neq j$ as depicted in Fig.1. 


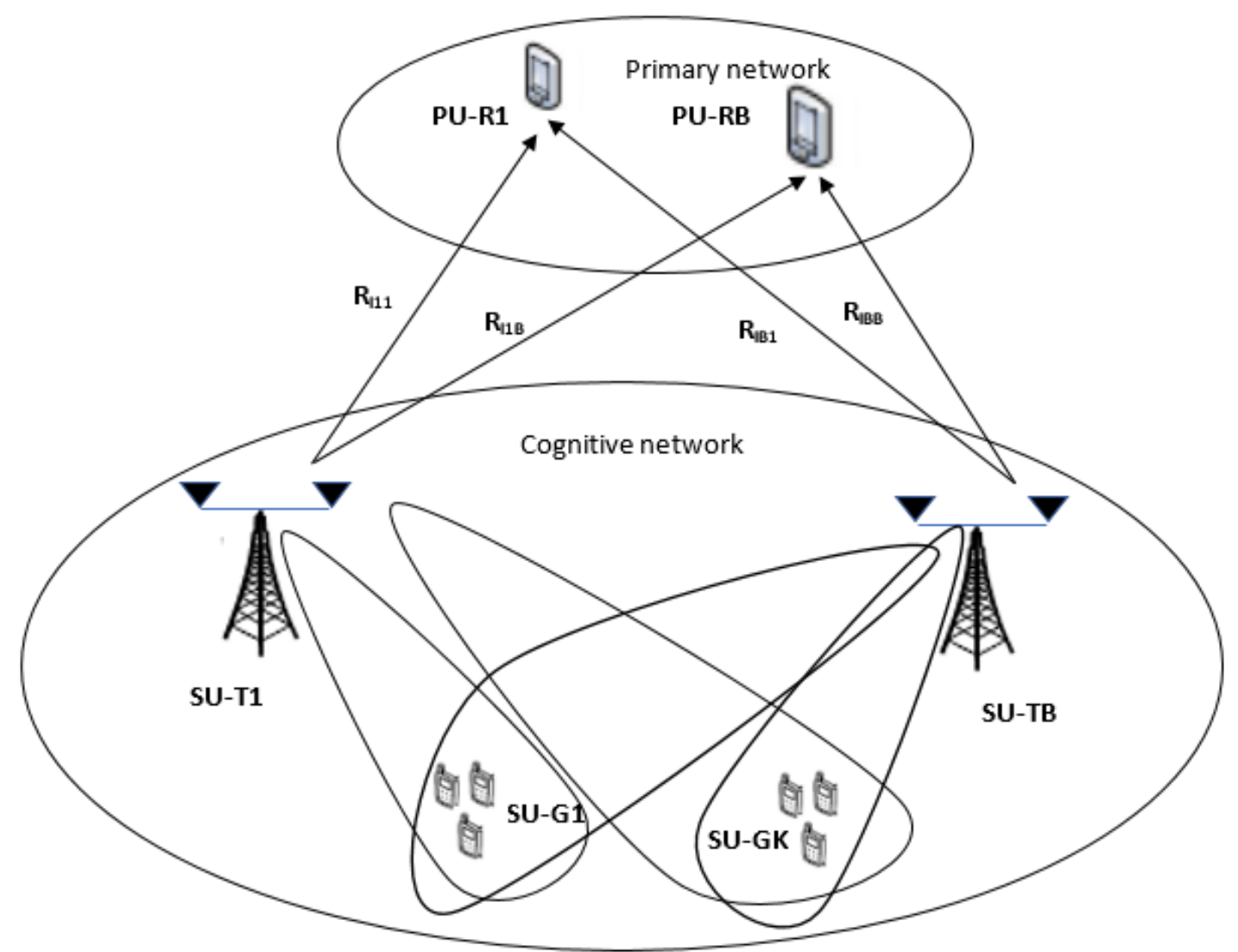

Figure 4.1: Cognitive Radio Multicast, Mulicell, Multigroup Wireless System Model

At each SU-T the signal intended to each group $\mathbf{s}_{i b}(t) \in \mathbb{C}^{N \times 1} \forall i \in[1, K]$ is superimposed with corresponding beamform vector $\mathbf{w}_{i b} \in \mathbb{C}^{N \times 1} \forall i \in[1, K]$ such that the total transmitted signal from each SU-T is $\mathbf{x}_{b}(t)=\sum_{i=1}^{K} \mathbf{s}_{i b}(t) \mathbf{w}_{i b} \in \mathbb{C}^{N \times 1}$ thus the concatenated signal from all base stations is $\mathbf{x}=\left[\mathbf{x}_{1}^{H}(t), \ldots, \mathbf{x}_{B}^{H}(t)\right]^{H} \in \mathbb{C}^{1 \times N B}$ The channel vector between each SU-T and SU-R is $\mathbf{h}_{b m} \in \mathbb{C}^{N \times 1} \forall m \in[1, \ldots, M]$ and $\forall b \in[1, \ldots, B]$ with corresponding channel vector between all SU-T and a single SU-R as the concatenation of individual channel vectors which is $\mathbf{h}_{m}=\left[h_{1 m}, \ldots, h_{B m}\right] \in$ $\mathbb{C}^{1 \times N B} \forall m \in[1, \ldots, M]$. Thus the received signal at the $m^{t h}$ user at time $t$ is given by: 


$$
\begin{gathered}
\mathbf{y}_{m}(t)=\mathbf{h}_{m}^{H} \mathbf{x}+n_{m} \\
\forall m \in(1, \ldots, M) \\
=\underset{\forall m \in(1, \ldots, M)}{\left[h_{1 m}, \ldots, h_{B m}\right]^{H}\left[\mathbf{x}_{1}^{H}(t), \ldots, \mathbf{x}_{B}^{H}(t)\right]^{H}+n_{m}}
\end{gathered}
$$

where $n_{m}$ is the zero-mean, circularly symmetric white Gaussian noise at the $m^{\text {th }}$ user.

Assume that the beamforming vector at the $m^{\text {th }}$ user is the concatenation of the beamforming vectors from all base stations which is $\mathbf{w}_{i}^{H}=\left[\mathbf{w}_{i 1}^{H}, \ldots, \mathbf{w}_{i B}^{H}\right] \in \mathbb{C}^{1 \times N B}$ $\forall i \in[1,, K]$. Define the covariance channel matrix at the $m^{\text {th }}$ user as $\mathbf{R}_{m} \in \mathbb{C}^{N B \times N B}$ which is either $\mathbf{R}_{m}=\mathbf{h}_{m} \mathbf{h}_{m}^{H}$ for a slow fading channel or $\mathbf{E}\left[\mathbf{h}_{m} \mathbf{h}_{m}^{H}\right]$ for a fast fading channel, and let $\sigma_{m}^{2}$ be the noise power. We can define the $S I N R_{m}$ at the $m^{t h}$ user when the received signal by each group $\mathbf{S}_{i}=\left[\mathbf{s}_{i 1}^{H}, \ldots, \mathbf{s}_{i B}^{H}\right]^{H} \in \mathbb{C}^{B N \times 1}$ satisfy$\operatorname{ing} \mathbf{S}_{i}^{H} \mathbf{S}_{i}=1 \forall i \in[1,, K]$ as:

$$
S I N R_{m}=\frac{\mathbf{w}_{k}^{H} \mathbf{R}_{m} \mathbf{w}_{k}}{\sum_{\substack{i=1, i \neq k \\ \forall m \in G_{k}, \forall k \in\{1, \ldots, K\}}}^{H} \mathbf{w}_{m} \mathbf{w}_{i}+\sigma_{m}^{2}}
$$

Each SU-T generates interference signal to the PU-R through channel matrix $\mathbf{h}_{I b b} \in \mathbb{C}^{N \times N_{P U}}$ which is given by:

$$
\mathbf{y}_{I b}(t)=\mathbf{h}_{\substack{I b \\ \forall b \in(1, \ldots, B)}}^{H}\left[\mathbf{x}_{1}^{H}(t), \ldots, \mathbf{x}_{B}^{H}(t)\right]^{H}
$$

where $\mathbf{h}_{I b}=\left[\mathbf{h}_{I 1 b} \ldots \mathbf{h}_{I B b}\right] \in \mathbb{C}^{N B \times N_{P U}} \forall b \in[1,, B]$ and $N_{P U}$ is the number of received antenna at the PU-R. 
Define $\mathbf{R}_{I b} \in \mathbb{C}^{N B \times N B}$ to be the interference covariance matrix between all SU-T and PU-R. The problem of maximizing the minimum SINR over all users can be written as:

$$
\begin{gathered}
(P 1): \\
\max _{\{\mathbf{w}\}_{k=1}^{K}} \min _{k \in\{1 \ldots \ldots K\}} \min _{m \in G_{k}} S I N R_{m} \\
\text { s.t. } \sum_{i=1}^{K}\left\|\mathbf{A}_{b} \mathbf{w}_{i}\right\|^{2} \leq P_{b}, \\
\forall b \in(1, \ldots, B) \\
\sum_{i=1}^{K} \mathbf{w}_{i}^{H} \mathbf{R}_{b I} \mathbf{w}_{i} \leq P_{I b}, \\
\forall b \in(1, \ldots, B)
\end{gathered}
$$

where $P_{b}$ is the maximum allowable transmitted power at the $b^{\text {th }} \mathrm{SU}-\mathrm{T}, P_{I b}$ is the maximal allowable interference power at PU-R $b$, and $\mathbf{A}_{b}=\operatorname{diag}\left\{\mathbf{e}_{\mathbf{b}}\right\} \otimes \mathbf{I}_{N}$.

As maintained previously, the CSI cannot be gathered perfectly at the receiver due to practical limitations and hence a full utilization of MISO and/or MIMO techniques advantages cannot be satisfied due to the effect of non-perfect CSI on system performances. This implies that the estimated covariance channel matrix is subject to uncertainty. Different uncertainty regions have been studied in prior research [81] In this work we will consider an uncertainty region that is bounded by the Frobenius norm, a commonly used approach. 
The relationship between the actual channel matrix and the estimated channel matrix that is subject to error is given by:

$$
\begin{gathered}
\mathbf{h}_{m}=\widehat{\mathbf{h}}_{m}+\delta_{m}, \forall m \in(1, \ldots, M) \\
\mathbf{h}_{I b}=\widehat{\mathbf{h}}_{I b}+\delta_{I b}, \forall b \in(1, \ldots, B)
\end{gathered}
$$

where $\widehat{\mathbf{h}}_{m}$ is the estimated channel vector between all the SU-T and single SU-R with $\delta_{\mathbf{m}}$ as the corresponding error, and $\widehat{\mathbf{h}}_{I b}$ the estimated channel vector between all the SU-T and single PU-R with $\delta_{\mathbf{I b}}$ as the corresponding error. Following some mathematical manipulation, the error in the covariance channel matrix for the secondary user can be written as.

$$
\begin{gathered}
\mathbf{R}_{m}=\widehat{\mathbf{R}}_{m}+\Delta \mathbf{R}_{m} \\
\left\|\Delta \mathbf{R}_{m}\right\|_{F} \leq \epsilon_{m} \\
\epsilon_{m}=S 1\left\|\widehat{\mathbf{R}}_{m}\right\|_{F} \\
\forall m \in(1, \ldots, M)
\end{gathered}
$$

Similarly for the interference channel is:

$$
\begin{gathered}
\mathbf{R}_{I b}=\widehat{\mathbf{R}}_{I b}+\Delta \mathbf{R}_{I b} \\
\left\|\Delta \mathbf{R}_{I b}\right\|_{F} \leq \epsilon_{I b} \\
\epsilon_{b}=S 2\left\|\widehat{\mathbf{R}}_{I b}\right\|_{F} \\
\forall b \in(1, \ldots, B)
\end{gathered}
$$

where $0 \leq S 1 \leq 1$ and $0 \leq S 2 \leq 1$, are a constants that represents the normalized uncertainty. 
Using (4.8) and (4.9) to represent deterministic error in the covariance channel matrix, channel problem $\mathrm{P} 1$ can be reduced to

$$
\begin{aligned}
& (P 2): \\
& \max _{\{\mathbf{w}\}_{k=1}^{K}} \min _{k \in\{1, \ldots, K\}} \min _{m \in G_{k}} S I N R_{m} \\
& \left\{\boldsymbol{\Delta} \mathbf{R}_{\mathbf{m}}\right\}_{m=1}^{M} \\
& \left\{\boldsymbol{\Delta} \mathbf{R}_{\mathbf{I b}}\right\}_{b=1}^{B} \\
& \text { s.t. } \widehat{\mathbf{R}}_{m}+\Delta \mathbf{R}_{m} \succeq 0 \\
& \left\|\Delta \mathbf{R}_{m}\right\|_{F} \leq \epsilon_{m} \\
& \sum_{i=1}^{K}\left\|\mathbf{A}_{b} \mathbf{w}_{i}\right\|^{2} \leq \mathbf{P}_{b} \\
& \sum_{i=1}^{K} \mathbf{w}_{i}^{H}\left(\mathbf{R}_{I b}+\Delta \mathbf{R}_{I} b\right) \mathbf{w}_{i} \leq P_{I b} \\
& \widehat{\mathbf{R}}_{I b}+\Delta \mathbf{R}_{I b} \succeq 0 \\
& \| \underset{\forall b \in(1, \ldots, B)}{\left\|\mathbf{R}_{I b}\right\|_{F} \leq \epsilon_{I b}}
\end{aligned}
$$

Problem P2 is difficult to solve due to the following two conditions:

1) problem $\mathrm{P} 2$ consists of multiple optimization variables $\left\{\boldsymbol{\Delta} \mathbf{R}_{\mathbf{I b}}\right\}_{b=1}^{B},\left\{\boldsymbol{\Delta} \mathbf{R}_{\mathbf{m}}\right\}_{m=1}^{M}$ and $\{\mathbf{w}\}_{k=1}^{K}$.

2) the objective function in P2 is fractional and non-convex and hence is difficult to optimize.

Using the procedure in $[85,88]$, one can introduce an auxiliary variable $\gamma \geq 0$ to replace the max min in the objective function with a single-level maximization objective via inclusion of new constraint (11b). This procedure allows us to reformulate 
problem P2 with the following equivalent representation:

$$
\begin{gathered}
(P 3): \\
\max _{\{\mathbf{w}\}_{k=1}^{K},\left\{\Delta \mathbf{R}_{m}^{M}\right\}_{m=1}^{M},\left\{\Delta \mathbf{R}_{I b}\right\}_{b=1}^{B}, \gamma} \gamma \\
\text { s.t.SIN } R_{m} \geq \gamma \\
\widehat{\mathbf{R}}_{m}+\Delta_{m} \succeq 0 \\
\left\|\mathbf{\Delta R}_{m}\right\|_{F} \leq \epsilon_{m} \\
\forall m \in G_{k}, \forall k \in\{1, \ldots, K\} \\
\sum_{k=1}^{K}\left\|\mathbf{A}_{b} \mathbf{w}_{k}\right\|^{2} \leq P_{b} \\
\sum_{i=1}^{K} \mathbf{w}_{i}^{H}\left(\widehat{\mathbf{R}}_{I b}+\Delta \mathbf{R}\right) \mathbf{w}_{i} \leq P_{I b}, \\
\widehat{\mathbf{R}}_{I b}+\Delta \mathbf{R}_{I b} \succeq 0 \\
\left\|\Delta \mathbf{R}_{I b}\right\|_{F} \leq \epsilon_{I b} \\
\forall b \in(1, \ldots, B)
\end{gathered}
$$

After reformulating P2 to P3, the objective function of P3 is convex but P3 consists of four optimization variables and one non-convex constraint (4.11b). Because Problem P3 is still difficult to analyze and solve, we thus its split it into two sub-problems. The first sub-problem consist of (4.11a),(4.11b),(4.11c), (4.11d) and (4.11e) which represents QOS for the secondary users problem. The second sub-problem consists of $(4.11 \mathrm{f}),(4.11 \mathrm{~g})$ and $(4.11 \mathrm{~h})$ which represents the interference power to the primary users. Each sub-problem is analyzed and solved separately, then their results are 
integrated to find an overall solution as illustrated in the following sections.

\subsection{Worst case approach}

In downlink beamforming in wireless system with CSI that are subject to error there are three types of solution in the literature, these solutions depend on the information available about the uncertainty matrices at the transmitter. The first method (Non-robust solution) assumes that the transmitter knows the uncertainty matrices perfectly and can insert its values into the beamforming problem. Although this solution has the best performance at the end user as compared with other two solutions, it has limited practical value due to the impractically of acquiring the perfect CSI error due to same causes of acquiring prefect CSI.

The second method, known as Sub-optimal solution, assumes that the uncertainty matrices are not known at the transmitter (neither its values nor the mathematical set to which it belongs). This solution has the worst performance as compared with the other two solutions, but has less complexity as compared with the third solution described in next section. This suboptimal solution solves for the worst case following the solution in $[78,86,87]$. here we will obtain the suboptimal solution using the SCA method as follows.

As discussed previously, we split p3 into two sub-problem, the first sub-problem consists of $(4.11 \mathrm{a}),(4.11 \mathrm{~b}),(4.11 \mathrm{c}),(4.11 \mathrm{~d})$ and $(4.11 \mathrm{e})$. The first constraint in this sub-

problem $(4.11 b)$ consists of multiple optimization variables $\left(\{\mathbf{w}\}_{k=1}^{K},\{\boldsymbol{\Delta} \mathbf{R}\}_{m=1}^{M}, \gamma\right)$ 
so that problem P3 cannot be solved as an optimization problem on a single variable. There are different approaches to solve this multivariate problem, and herein we will illustrate the conservative approach. More precisely, for a conservative solution, we obtain a worst case solution for the non-convex term (4.11b) with respect to $\{\boldsymbol{\Delta} \mathbf{R}\}_{m=1}^{M}$ by minimizing the term (replacing it by its lower bound with respect to $\left.\{\boldsymbol{\Delta} \mathbf{R}\}_{m=1}^{M}\right)$. This can be accomplished by replacing the numerator by its minimum and the denominator by its maximum and following the procedure in [120], the constraints $,(4.11 b),(4.11 \mathrm{c}),(4.11 \mathrm{~d})$ and $(4.11 \mathrm{e})$ can be represented by the equivalent constraint : constraint :

$$
\mathbf{w}_{k}^{H}\left(\widehat{\mathbf{R}}_{m}-\epsilon_{\mathbf{m}} \mathbf{I}_{\mathbf{L}}\right)^{+} \mathbf{w}_{k} \geq \gamma\left(\sum_{\substack{i=1, i \neq k \\ \forall m \in G_{k}, \forall k \in\{1, \ldots, K\}}}^{K} \mathbf{w}_{i}^{H}\left(\widehat{\mathbf{R}}_{m}+\epsilon_{\mathbf{m}} \mathbf{I}_{\mathbf{L}}\right) \mathbf{w}_{i}\right)
$$

where $\mathrm{L}$ is the dimension of covariance matrix $\mathrm{R}$.

The second sub-problem consists of (4.11f), and (4.11g):

$$
\begin{gathered}
\max _{\{\mathbf{w}\}_{k=1}^{K},\left\{\mathbf{\Delta} \mathbf{R}_{I b}\right\}_{b=1}^{B}} \sum_{i=1}^{K} \mathbf{w}_{i}^{H}\left(\widehat{\mathbf{R}}_{I b}+\Delta \mathbf{R}_{I b}\right) \mathbf{w}_{i} \leq P_{I b}, \\
\underset{\forall b \in(1, \ldots, B), \forall m \in G_{k}, \forall k \in\{1, \ldots, K\}}{\widehat{\mathbf{R}}_{I b}+\Delta \mathbf{R}_{I b} \succeq 0}
\end{gathered}
$$

The uncertainty set is assumed such that its Frobenius norm does not exceed a maximum-allowable value $\epsilon_{I b}[35]$. The objective in this case is to find a value for $\Delta \mathbf{R}_{I b}$ that maximizes the objective function in (4.13), this can be accomplished by replacing $\Delta \mathbf{R}_{I b}$ by $\epsilon_{I b} \mathbf{I}$ so we have two cases:

1) $\mathbf{R}_{I b} \geq \epsilon_{I b} \mathbf{I}$ : In this case by the definition of quadratic form [98] we have 


$$
\chi^{H}\left(\mathbf{R}_{I b}+\epsilon_{I b} \mathbf{I}_{L B}\right) \chi \geq \chi^{H}\left(\mathbf{R}_{I b}-\epsilon_{I b} \mathbf{I}_{L B}\right) \chi
$$

Thus $\left(\mathbf{R}_{I b}+\epsilon_{I b} \mathbf{I}_{L B}\right)$ is adequate to choose to maximize the value of (4.18a)

2) $\mathbf{R}_{I b} \leq \epsilon_{I b} \mathbf{I}$ : in this case and by contradiction the resultant $\mathbf{R}_{I b}-\epsilon_{I b}$ is Hermitian (not positive semidefinite(PSD)). But this would violate the definition of a covariance channel matrix which is always PSD, and so in this case we can replace $\mathbf{R}_{I b}+\Delta \mathbf{R}$ by $\mathbf{R}_{I b}+\epsilon_{I b} \mathbf{I}_{L B}$; thus(4.13) is replaced with its equivalent:

$$
\sum_{i=1}^{K} \mathbf{w}_{i}^{H}\left(\widehat{\mathbf{R}}_{I b}+\epsilon_{I b} \mathbf{I}_{I b}\right) \mathbf{w}_{i} \leq P_{I b},
$$

Substituting (4.12) and(4.15) into P3 we now have two optimization variables $(\gamma$ and $\mathbf{w}_{i}$ ). While P3 is not convex, using the SCA beamforming method [85] and replacing $\gamma$ with $\gamma^{(t)}+\Delta \gamma$ and $\mathbf{w}_{i}$ with $\mathbf{w}_{i}^{(t)}+\Delta \mathbf{w}_{i}$, the problem can be transformed into:

$(P 4):$

$$
\begin{gathered}
\max _{\{\Delta \mathbf{w}\}_{k=1}^{K}, \Delta \gamma} \Delta \gamma \\
\text { s.t. }-\left(\mathbf{w}_{k}^{(t) H}\left(\widehat{\mathbf{R}}_{m}-\epsilon_{m} \mathbf{I}_{m}\right)^{+} \mathbf{w}_{k}^{(t)}+2 \Re\left(\Delta \mathbf{W}_{k}^{H}\left(\widehat{\mathbf{R}}_{m}-\epsilon_{m} \mathbf{I}_{m}\right)^{+} \mathbf{w}_{k}^{(t)}\right)\right) \\
+\gamma^{(t)}\left(\sum_{i=1, i \neq k}^{K}\left(\mathbf{w}_{i}^{(t) H}+\Delta \mathbf{w}\right)\left(\widehat{\mathbf{R}}_{m}+\epsilon_{m} \mathbf{I}_{m}\right)\left(\mathbf{w}_{k}^{(t)}+\Delta \mathbf{w}\right)\right)
\end{gathered}
$$




$$
\begin{gathered}
+\Delta \gamma\left(\sum_{i=1, i \neq k}^{K} \mathbf{w}_{k}^{(t) H}\left(\widehat{\mathbf{R}}_{m}+\epsilon_{m} \mathbf{I}_{m}\right) \mathbf{w}_{k}^{(t)}\right) \\
+\sigma_{m}^{2}\left(\Delta \gamma+\gamma^{(t)}\right) \leq 0 \\
\sum_{i=1}^{K}\left\|\mathbf{A}_{b}\left(\Delta \mathbf{w}_{i}+\mathbf{w}_{i}^{(t)}\right)\right\|^{2} \leq \mathbf{P}_{b} \\
\sum_{i=1}^{K}\left(\Delta \mathbf{w}_{i}+\mathbf{w}_{i}^{(t)}\right)^{H}\left(\widehat{\mathbf{R}}_{I b}+\epsilon_{I b} \mathbf{I}_{I b}\right)\left(\Delta \mathbf{w}_{i}+\mathbf{w}_{i}^{(t)}\right) \leq P_{I b}, \\
\forall m \in G_{k}, \forall k \in\{1, \ldots, K\}, \forall b \in(1, \ldots, B)
\end{gathered}
$$

We can optimize P4 by using an iterative approach, in which we start with random values for $\mathbf{w}_{i}^{(t)}$ and $\gamma^{(t)}$ and then update iteratively as follows.

$$
\begin{gathered}
\mathbf{w}_{i}^{(t+1)}=\mathbf{w}_{i}^{(t)}+\Delta \mathbf{w}_{i} \\
\gamma^{(t+1)}=\gamma^{(t)}+\Delta \gamma
\end{gathered}
$$

The iteration is stopped when $\Delta \gamma$ approaches some small predefined value or the number of iterations exceeds a prespecified maximum.

The positive exponent in the first and second terms of the first constraint in $\mathrm{p} 4$ means that $\left(\widehat{\mathbf{R}}_{m}-\epsilon_{m} \mathbf{I}_{m}\right)^{+} \succeq 0$ (PSD), this can be ensured by replacing every negative eigenvalue of $\left(\widehat{\mathbf{R}}_{m}-\epsilon_{m} \mathbf{I}_{m}\right)$ by zero

For the solution to be feasible and convergence to be assured the following two conditions must hold: 
1) $\left(\widehat{\mathbf{R}}_{m}-\epsilon_{m} \mathbf{I}_{m}\right)$ is PSD

2) $\Delta \gamma$ is greater than zero.

For more details of the feasibility and the convergence of the P4 please refer to Appendix 1

The complexity of the SCA in terms of the system parameters (number of transmit antenna in each base station, number of receive antenna in each user, number of groups and, number of users in each group) is given by $\mathcal{O}\left((M+1)^{1 / 2}(M+K N+2)(K N+1)^{2}\right)$

\subsection{Robust solution}

The SCA method can be invoked to find the suboptimal solution. In this section we will find the robust beamforming solution and mitigate the effect of channel error. In beamforming literature, the robust solution outperforms the suboptimal solution. In this robust solution the uncertainty matrix coefficients are assumed to be unknown, but its mathematical set is known. We return to p3 to explain this solution.

Problem P3 consist of four optimization variables as discussed previously and is therefore difficult to solve as a single optimization problem. Thus, it is partitioned into two sub-optimization problems as in section 3. The first optimization problem is that considering the interference power to the primary user:

$$
\begin{gathered}
\max _{\{\mathbf{w}\}_{k=1}^{K},\left\{\boldsymbol{\Delta} \mathbf{R}_{I b}\right\}_{b=1}^{B}} \sum_{i=1}^{K} \mathbf{w}_{i}^{H}\left(\widehat{\mathbf{R}}_{I b}+\Delta \mathbf{R}_{I b}\right) \mathbf{w}_{i} \\
\widehat{\mathbf{R}}_{I b}+\boldsymbol{\Delta} \mathbf{R}_{I b} \succeq 0
\end{gathered}
$$




$$
\begin{gathered}
\left\|\boldsymbol{\Delta} \mathbf{R}_{I b}\right\|_{F} \leq \epsilon_{I b} \\
\forall b \in(1, \ldots, B), \forall m \in G_{k}, \forall k \in\{1, \ldots, K\}
\end{gathered}
$$

Using the definition of quadratic form for positive (semi)definite P(S)D and negative (semi)definite $\mathrm{N}(\mathrm{S}) \mathrm{D}$ and assuming $\Delta \mathbf{R}_{I b}^{1}$ is $\mathrm{P}(\mathrm{S}) \mathrm{D}$ while $\Delta \mathbf{R}_{I b}^{2}$ is $\mathrm{N}(\mathrm{S}) \mathrm{D}$. we always know that $\widehat{\mathbf{R}}_{I b}$ is PSD, and thus:

$$
\mathbf{w}_{k}^{H}\left(\widehat{\mathbf{R}}_{I b}+\underset{\forall m \in G_{k}, \forall k \in\{1, \ldots, K\}}{\left.\Delta \mathbf{R}_{I b}^{2}\right) \mathbf{w}_{k}<\mathbf{w}_{k}^{H}\left(\widehat{\mathbf{R}}_{I b}+\Delta \mathbf{R}_{I b}^{1}\right) \mathbf{w}_{k}}\right.
$$

The resultant matrix of maximization of any quadratic form is always $\mathrm{P}(\mathrm{S}) \mathrm{D}$ Then (4.18) is reduced to:

$$
\begin{gathered}
\min _{\{\mathbf{w}\}_{k=1}^{K},\left\{\mathbf{\Delta} \mathbf{R}_{I b}\right\}_{b=1}^{B}}-\sum_{i=1}^{K} \mathbf{w}_{i}^{H}\left(\widehat{\mathbf{R}}_{I b}+\Delta \mathbf{R}\right) \mathbf{w}_{i}, \\
\left\|\mathbf{\Delta} \mathbf{R}_{I b}\right\|_{F}^{2} \leq \epsilon_{I b}^{2} \\
\forall b \in(1, \ldots, B), \forall m \in G_{k}, \forall k \in\{1, \ldots, K\}
\end{gathered}
$$

Problem (4.20) is thus convex and the equivalent Lagrangian function after defining $\lambda_{I b}$ as a Lagrange multiplier is:

$$
\begin{gathered}
\inf _{\Delta \mathbf{R}_{i b}} f\left(\Delta \mathbf{R}_{I b}, \lambda_{I b}\right)=-\sum_{i=1}^{K} \mathbf{w}_{i}^{H}\left(\widehat{\mathbf{R}}_{I b}+\Delta \mathbf{R}\right) \mathbf{w}_{i}, \\
+\lambda_{i b}\left(\left\|\Delta \mathbf{R}_{I b}\right\|_{F}^{2}-\epsilon_{I b}^{2}\right) \\
\forall b \in(1, \ldots, B), \forall m \in G_{k}, \forall k \in\{1, \ldots, K\}
\end{gathered}
$$

To find the optimal value of $\Delta \mathbf{R}_{I b}$ we can differentiate (4.21) and equate to zero

$$
\Delta \mathbf{R}_{I b}=\frac{\sum_{i=1}^{K}\left\|\mathbf{w}_{i}\right\|_{F}^{2}}{2 \lambda_{I b}}
$$

substituting (4.22) into (4.21) leads to 


$$
f\left(\lambda_{I b}\right)=-\frac{\sum_{i=1}^{K}\left\|\mathbf{w}_{i}\right\|_{F}^{4}}{4 \lambda_{I b}}-\lambda_{I b} \epsilon^{2}-\sum_{i=1}^{K} \mathbf{w}_{i} \widehat{\mathbf{R}}_{I b} \mathbf{w}_{k}
$$

The corresponding dual problem is

$$
\begin{gathered}
\min _{\lambda_{I b}}=-\frac{\sum_{i=1}^{K}\left\|\mathbf{w}_{i}\right\|_{F}^{4}}{4 \lambda_{I b}}-\lambda_{I b} \epsilon^{2}-\sum_{i=1}^{K} \mathbf{w}_{i} \widehat{\mathbf{R}}_{I b} \mathbf{w}_{k} \\
\text { s.t } \lambda_{I b} \geq 0 \\
\forall b \in(1, \ldots, B), \forall m \in G_{k}, \forall k \in\{1, \ldots, K\}
\end{gathered}
$$

To find the optimal value of $\lambda_{I b}$, we can differentiate (??) with respect to $\lambda_{I b}$ and equate to zero obtaining

$$
\begin{gathered}
\lambda_{I b}=\frac{\sum_{i=1}^{K}\left\|\mathbf{w}_{i}\right\|_{F}^{2}}{2 \epsilon_{I b}} \\
\forall b \in(1, \ldots, B), \forall m \in G_{k}, \forall k \in\{1, \ldots, K\}
\end{gathered}
$$

When substituting (4.21) in (??) we obtain.

$$
\sum_{i=1}^{K}\left(\mathbf{w}_{i}^{H} \widehat{\mathbf{R}}_{I b} \mathbf{w}_{i}+\epsilon_{I b}\left\|\mathbf{w}_{i}\right\|_{F}^{2}\right) \leq P_{I b}
$$

Which is the optimal solution for the interference power problem which can be scholastically proven by defining. 


$$
\operatorname{Pr}_{I b}\left(\sum_{i=1}^{K} \mathbf{w}_{i}^{H}\left(\widehat{\mathbf{R}}_{I b}+\Delta \mathbf{R}_{I b}\right) \mathbf{w}_{i} \leq P_{I b},\right) \geq \text { thre }_{I b}
$$

where $\operatorname{Pr}_{I b} \sum_{i=1}^{K} \mathbf{w}_{i}^{H}\left(\widehat{\mathbf{R}}_{I b}+\Delta \mathbf{R}_{I b}\right) \mathbf{w}_{i} \leq P_{I b}$ is the probability that $\sum_{i=1}^{K} \mathbf{w}_{i}^{H}\left(\widehat{\mathbf{R}}_{I b}+\right.$ $\left.\Delta \mathbf{R}_{I b}\right) \mathbf{w}_{i}$ with respect to $\Delta \mathbf{R}_{I b}$ less than $P_{I b} \forall b \in(1, \ldots, B)$ is grater than predefined threshold $\left(\right.$ thre $\left._{I b}\right)$ which is less than or equal one.

The probability value can be found using the definition of normal distribution with mean $\mu_{I b}$ and variance $v a r_{I b}$

$P r_{I b}=\frac{1}{\sqrt{2 \pi v a r_{I b}}} \int_{-\infty}^{P_{I b}} \frac{E X P\left(Y_{I b}-\mu_{I b}\right)}{2 v a r_{I b}} d Y_{I b}(4.24)$

the solution of above integral is given by:

The solution of above integral is given by:

$$
\operatorname{Pr}_{I b}= \begin{cases}\frac{1}{2}-\frac{1}{2} \operatorname{erf}\left(\frac{\mu_{I b}-P_{I b}}{\sqrt{2} v_{I b}}\right) & \text { if } P_{I b} \leq \mu_{I b} \\ \frac{1}{2}+\frac{1}{2} \operatorname{erf}\left(\frac{P_{I b}-\mu_{I b}}{\sqrt{2} \operatorname{var}_{I b}}\right) & \text { if } P_{I b} \geq \mu_{I b}\end{cases}
$$

The value of mean $\mu_{I b}$ and variance $\operatorname{var}_{I b}$ can be proven to be $\sum_{i=1}^{K} \mathbf{w}_{i}^{H} \widehat{\mathbf{R}}_{I b} \mathbf{w}_{i}$ and $\beta_{I b} \sum_{i=1}^{K}\left\|w_{i}\right\|_{F}^{2}$ respectively where $0 \leq \beta_{I b} \leq 1$

Substituting the values of mean and variance into (4.25), after several mathematical manipulations the statistical solution of interference power is reduced to:

$$
\sum_{i=1}^{K}\left(\mathbf{w}_{i}^{H} \widehat{\mathbf{R}}_{I b} \mathbf{w}_{i}+\alpha_{I b}\left\|\mathbf{w}_{i}\right\|_{F}^{2}\right) \leq P_{I b}
$$

where $\alpha_{I b}=\sqrt{2 \beta_{I b}} \operatorname{erf}^{-1}\left(P_{I b}-1\right) \forall b \in(1, \ldots, B)$ and thus (4.22) and (4.26) are equiv- 
alent mathematically.

The second optimization problem considers the QOS at the secondary users, which consists of $(4.11 \mathrm{a}),(4.11 \mathrm{~b}),(4.11 \mathrm{c}),(4.11 \mathrm{~d})$ and $(4.11 \mathrm{e})$ in addition to (4.22). This problem consists of three optimization variables $\left(\gamma, \boldsymbol{\Delta} \mathbf{R}_{m}\right.$, and $\left.\mathbf{w}_{i}\right)$, and is difficult to solve as a single optimization problem. Here we solve this problem using an alternate-iterate optimization technique. In particular, our approach holds one (two) variable(s) fixed and finds a solution for the other variable(s) at each iteration. This technique is illustrated as follows.

1)First we find the solution for $\{\boldsymbol{\Delta} \mathbf{R}\}_{m=1}^{M}$ by assuming that $\mathbf{w}_{i}$ and $\gamma$ are known, thus the QOS problem is reduced to :

$$
\begin{aligned}
& \text { (P5) : } \\
& \max _{\{\Delta \mathbf{R}\}_{m=1}^{M}} \gamma \sum_{i=1, i \neq k}^{K} \mathbf{w}_{i}^{H} \Delta \mathbf{R}_{m} \mathbf{w}_{i}-\mathbf{w}_{k}^{H} \Delta \mathbf{R}_{m} \mathbf{w}_{k} \\
& \text { s.t } \widehat{\mathbf{R}}_{m}+\Delta \mathbf{R}_{m} \succeq 0 \\
& \underset{\forall m \in \mathbf{R}_{m}, \forall k \in\{1, \ldots, K\}}{\forall} \|_{F} \leq \epsilon_{m}
\end{aligned}
$$

P5 is a convex in $\Delta \mathbf{R}_{m}$ and can be solved using a convex optimization package such as CVX [122] or following our previous procedure in [120] for more detail see appendix 2 . 
2) We then find a solution for $\{\mathbf{w}\}_{k=1}^{K}$, inserting the solution for $\Delta \mathbf{R}_{m}$ returned from 1 and the solution of $\Delta \mathbf{R}_{I b}$ into P3, we can reduce P3 to:

$$
\begin{gathered}
(P 6): \\
\max _{\{\mathbf{w}\}_{k=1}^{K}, \gamma} \gamma \\
\text { s.t. } \gamma\left(\sum_{i=1, i \neq k}^{K} \mathbf{w}_{i}^{H}\left(\widehat{\mathbf{R}}_{m}+\Delta \mathbf{R}_{m}\right) \mathbf{w}_{i}+\sigma_{m}^{2}\right) \\
-\mathbf{w}_{k}^{H}\left(\widehat{\mathbf{R}}_{m}+\Delta \mathbf{R}_{m}\right) \mathbf{w}_{k} \leq 0 \\
\left\|\mathbf{A}_{b} \mathbf{w}_{k}\right\|^{2} \leq \mathbf{P}_{b} \\
\sum_{i=1}^{K}\left(\mathbf{w}_{i}^{H} \widehat{\mathbf{R}}_{I b} \mathbf{w}_{i}+\epsilon_{I b}\left\|\mathbf{w}_{i}\right\|_{F}^{2}\right) \leq P_{I b} \\
\forall m \in G_{k}, \forall k \in(1, \ldots, K), \forall b \in(1, \ldots, B)
\end{gathered}
$$

Due to the non-convexity of constraint $(4.28 \mathrm{~b}) \mathrm{m}$ problem P6 is not convex. The problem can be solved using the SCA method as in the previous section (similar to [120]) after adding the solution of the interference power to the primary users where the algorithm can be modified to solve the problem. Here we will illustrate the solution using bisection search method.

Problem P6 can be reformulated to be solved using bisection search method after inserting the values of $\Delta \mathbf{R}_{m}$ and $\Delta \mathbf{R}_{I b}$ as follows:

$(P 7)$ : 


$$
\begin{gathered}
\max _{\{\mathbf{w}\}_{k=1}^{K}} \min _{k \in\{1, \ldots, K\}} \min _{m \in G_{k}} \frac{\left\|\mathbf{w}_{k}\right\|^{2}\left|\left(\widehat{\mathbf{R}}_{m}+\mathbf{\Delta} \mathbf{R}_{m}\right)\right|}{\left|\left(\widehat{\mathbf{R}}_{m}+\mathbf{\Delta} \mathbf{R}_{m}\right)\right| \sum_{i=1, i \neq k}^{K}\left\|\mathbf{w}_{k}\right\|^{2}(4.29 \mathrm{a})} \\
\text { s.t. } \sum_{k=1}^{K}\left\|\mathbf{A}_{b} \mathbf{w}_{k}\right\|^{2} \leq \mathbf{P}_{b} \\
\sum_{i=1}^{K}\left(\mathbf{w}_{i}^{H} \widehat{\mathbf{R}}_{I b} \mathbf{w}_{i}+\epsilon_{I b}\left\|\mathbf{w}_{i}\right\|_{F}^{2}\right) \leq P_{I b} \\
\forall m \in G_{k}, \forall k \in(1, \ldots, K), \forall b \in(1,, \ldots, B)
\end{gathered}
$$

Problem P7 cannot be solved using a convex optimization package. Instead, we solve it using the bisection search method after defining $\psi$ as an auxiliary variable as follows:

$$
\begin{gathered}
(P 8): \\
\min _{\{\mathbf{w}\}_{k=1}^{K} .} \sum_{k=1}^{K}\left\|\mathbf{A}_{b} \mathbf{w}_{k}\right\|^{2} \\
\text { s.t. }\left\|\mathbf{w}_{k}\right\|^{2}\left|\left(\widehat{\mathbf{R}}_{m}+\Delta \mathbf{R}_{m}\right)\right| \geq \psi\left|\left(\widehat{\mathbf{R}}_{m}+\Delta \mathbf{R}_{m}\right)\right| \sum_{i=1, i \neq k}^{K}\left\|\mathbf{w}_{i}\right\|^{2}(4.30 b) \\
\sum_{i=1}^{K}\left(\mathbf{w}_{i}^{H} \widehat{\mathbf{R}}_{I b} \mathbf{w}_{i}+\epsilon_{I b}\left\|\mathbf{w}_{i}\right\|_{F}^{2}\right) \leq P_{I b} \\
\forall m \in G_{k}, \forall k \in(1, \ldots, K), \forall b \in(1, \ldots, B)
\end{gathered}
$$

Algorithm 1 provides details of the robust solution using bisection search method where the solution alternates iteratively in solving the problem. The complexity of bisection search in term of system parameters is $\mathcal{O}\left(K^{3} N^{6}+M K N^{2}\right)$ [118].

The NMSPG method is a convex optimization method that can be used to solve P9(see Appendix 2) and is of linear complexity. the method is available in common convex optimization packages such as CVX [122], for more details see [121]

Combining NMSPG method with the SCA method gives a total complexity of 
$\left.\left(3 N+\mathcal{O}(1)+\mathcal{O}\left((M+1)^{(1 / 2)}(M+K N+2)\left(K N+1^{2}\right)\right)\right)\right)$. Alternatively, the combined NMSPG with bisection search method gives a total complexity of $(3 N+\mathcal{O}(1)+$ $\mathcal{O}\left(K^{3} N^{6}+M K N^{2}\right)$

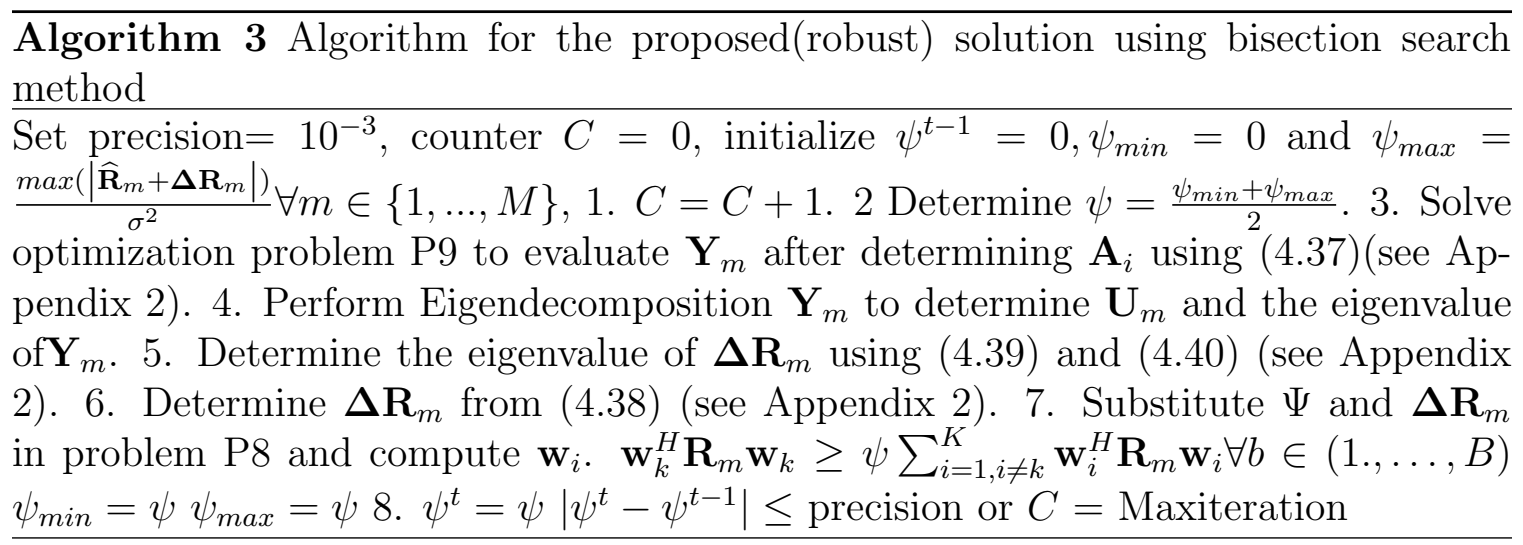

\subsection{Simulation results}

Simulation results are presented in this section to illustrate the existence and convergence of our methodology using different system parameters. The estimated channel matrices of the secondary users $\widehat{\mathbf{h}}_{m} \in \mathbb{C}^{1 \times N} \forall m \in[1, \ldots, M]$, the estimated interference channel matrices $\widehat{\mathbf{h}}_{I b} \in \mathbb{C}^{N B \times N_{p u}} \forall b \in[1, \ldots, B]$ and the initial beamforming vector $\mathbf{w}_{i} \in \mathbb{C}^{N \times 1} \forall i \in[1, \ldots, K]$ are randomly generated from a complex zero-mean circularly Gaussian distribution with unit variance. The estimated covariance secondary users and interference channel matrices are determined assuming a slow fading channel i.e $\widehat{\mathbf{R}}_{m}=\widehat{\mathbf{h}}_{m} \widehat{\mathbf{h}}_{m}^{H}$ and $\widehat{\mathbf{R}}_{I b}=\widehat{\mathbf{h}}_{I b} \widehat{\mathbf{h}}_{I b}^{H}$. A precision of $10^{-3}$ is assumed throughout the simulation in order to demonstrate the results of four type of solutions:

1) Robust solution using SCA method.

2) Robust solution using bisection search method.

3) Sub-optimal solution. 
4) Non-Robust solution.

A set of 500 Monte-Carlo runs are averaged for each simulation study throughout this work.

In the first simulation, shown in Figure 2, we assume that the system consists of two base stations at $\mathrm{SU}-\mathrm{T}$ each having five antennas $(\mathrm{N}=5)$ that utilize the frequency resources of two five antennas PU-R $\left(N_{p u}=5\right)$, there are twelve SU-R $(\mathrm{M}=12)$ that are partitioned into two multicast groups $(K=2)$, the channel uncertainty percentage at the second user's channel and interference channel are assumed to be 10 percent $\epsilon_{m}=\epsilon_{I b}=.01$, the maximum transmitted total power from all SU-T is bounded to be not more than $20 \mathrm{~dB}\left(P_{b}=100\right)$ and the maximum allowable interference power at the PU-R is $-5 \mathrm{~dB}$.

In our previous work [120] we showed that the SCA method outperform the QOS, the suboptimal and the non-robust solutions for different numbers of groups within specified number of users. Here it is found that that the SCA method has a better convergence speed than the bisection search method as shown in Figure 2, however when the number of iterations increases, then the latter outperform the other three method(SCA, sub-optimal and non-robust) in term of system performance as represented by minimum SINR. This can offer a good tradeoff between the high speed of convergence that can be obtained using the SCA method and the best QOS at the SU-R which is achieved using the bisection search method. 


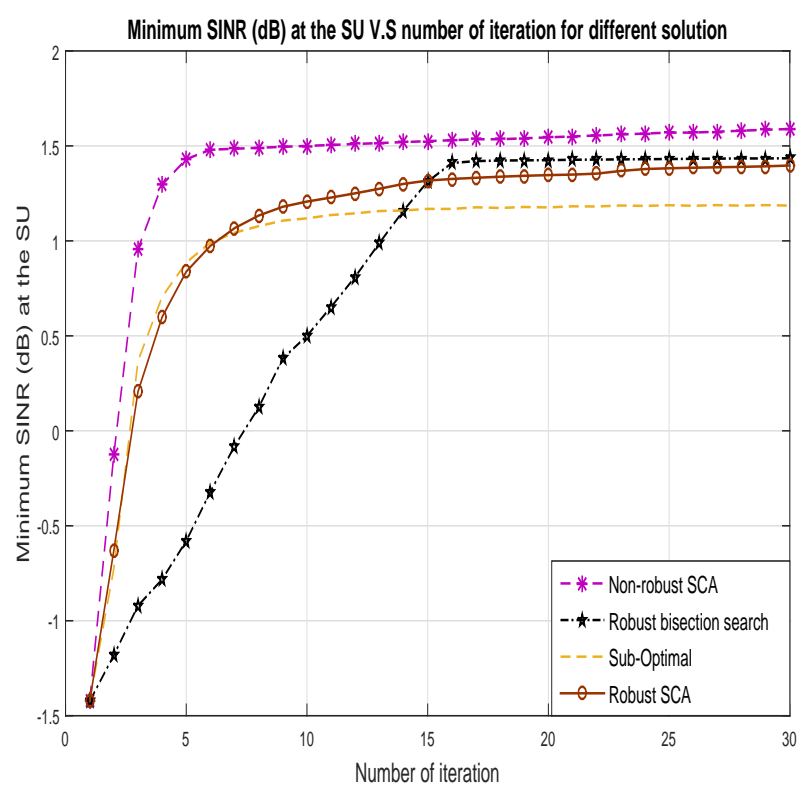

Figure 4.2: Minimum SINR for different solutions versus number of iteration $\mathbf{N}=$ $\mathbf{N}_{p u}=5, \mathbf{M}=12, \mathbf{k}=2, \sigma_{m}=1, \epsilon_{m}=\epsilon_{I b}=.01, P_{I P}$ and $P_{b}=20 d B$

In the second example shown in Figure 3 and, for the same system parameters used in the first simulation and assuming that all the users have the same minimum SINR, which is predefined for the range between $(-5-10) \mathrm{dB}$, we determine the percentage feasibility, that is the ratio between the number of SU-R users that satisfy the minimum SINR to the number of all SU-R users(M). We find that that the SCA and bisection methods outperform the rank one, rank two and sub-optimal solutions. 


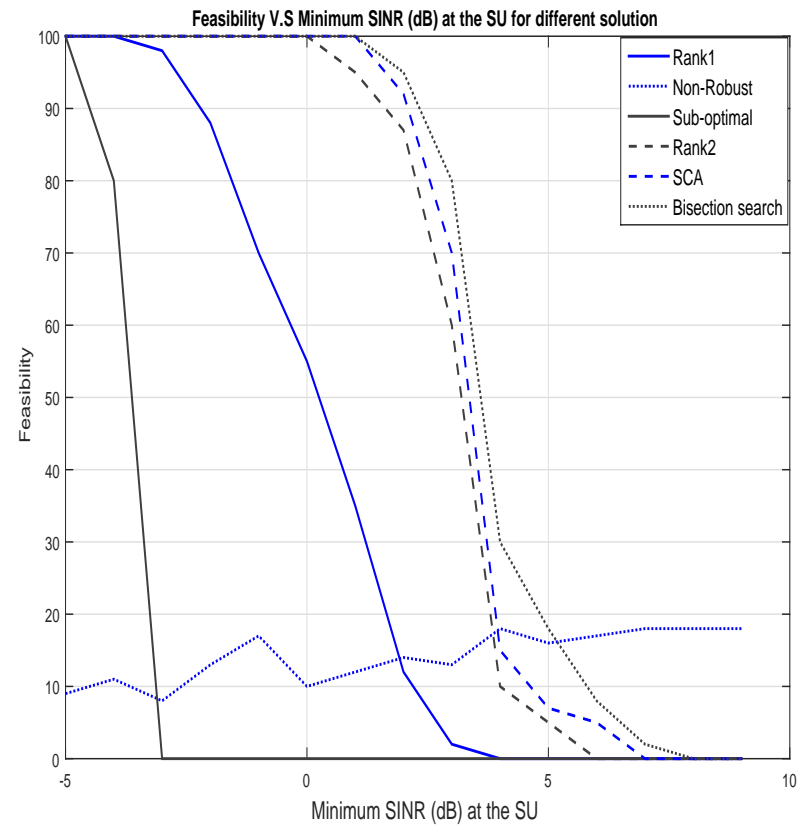

Figure 4.3: $\quad$ Minimum SINR for different solutions versus feasibility

In the third example we demonstrate the effect of the number of users $(\mathrm{M}=[1-$ 15]) on the minimum SINR at the SU-R as depicted in Figure 4, and find that as the number of users(M) increases the minimum SINR decreases, due to the fact that the power transmitted by the SU-T base stations is distributed among all the users. Also, as the secondary user channel uncertainty $\epsilon_{m}$ increases the received minimum SINR by the SU-R decreases. 


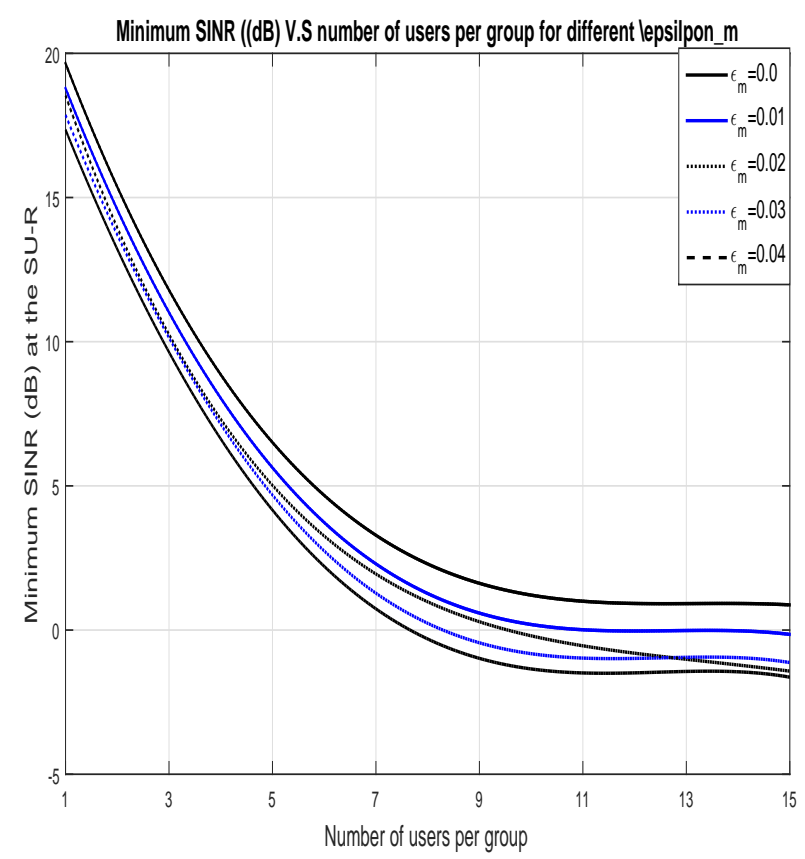

Figure 4.4: Minimum SINR for different uncertainty ratio $\epsilon_{m}$ solutions versus number of users per group

The fourth simulation shown in Figure 5 examine the effect of the total minimum transmitted power by the SU-T for different solutions (SCA, Bisection search and Non-robust) and different secondary user channel uncertainty $\left(\epsilon_{m}\right)$ on the minimum received SINR at the SU-R users. The figure indicates that the bisection search method outperform both the SCA method and the non-robust solution for the same value of $\epsilon_{m}$ and also suggests that the SCA method is more power efficient than the non-robust solution. This is true due to its relation to the percentage feasibility sequences of the three solution. Also, as the value of the uncertainty $\left(\epsilon_{m}\right)$ increases the required transmitted power to achieve the same minimum SINR reduces. 


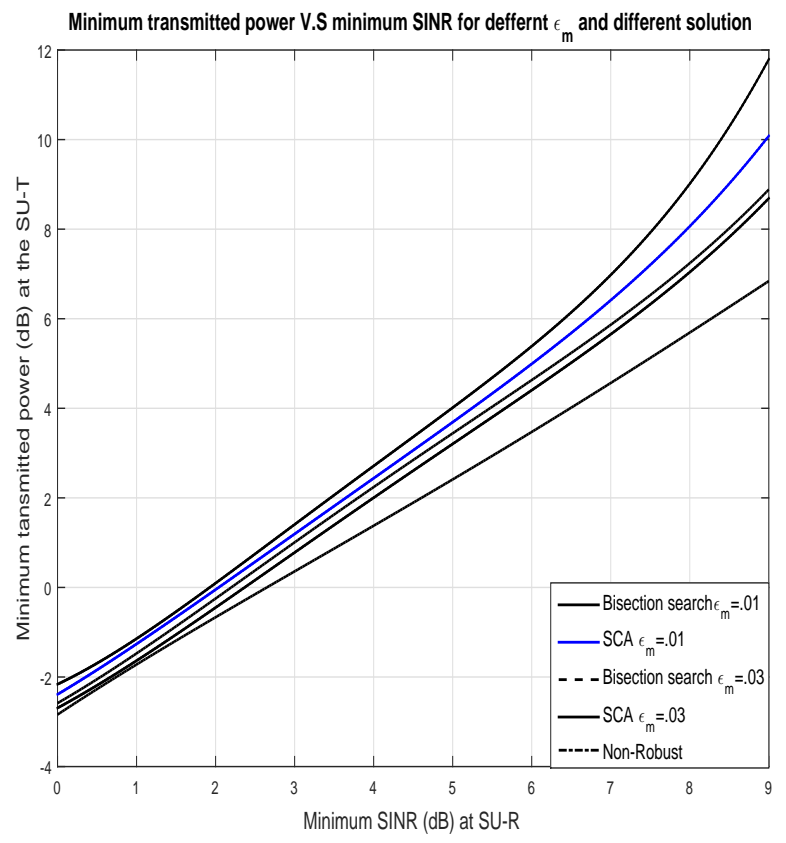

Figure 4.5: Minimum SINR for different solutions and different uncertainty radios $\epsilon_{m}$ versus minimum transmitted power

Finally Figures 6 and 7 shows the cumulative distribution function (CDF) of the interference power at the PU-R and the minimum SINR at the SU-R, respectively. This analysis finds that the SCA and bisection search methods are more efficient than the non-robust solution in reducing the interference power toward the PU-R and for increasing the amount of minimum SINR at the SU-R. 


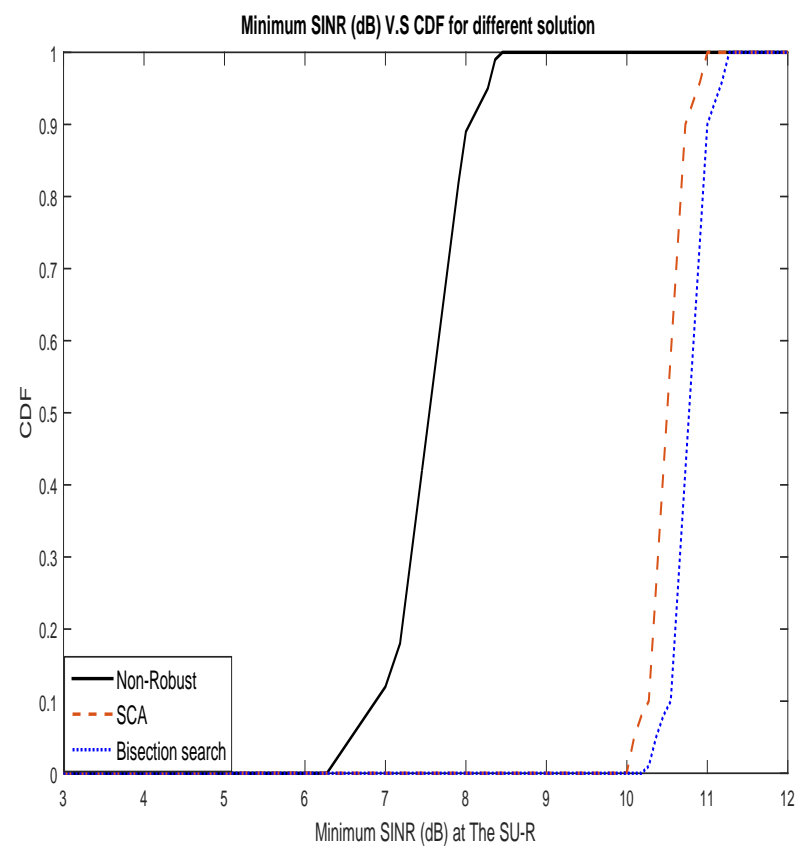

Figure 4.6: Minimum SINR for different solutions versus cumulative distribution function (CDF) at SU-R 


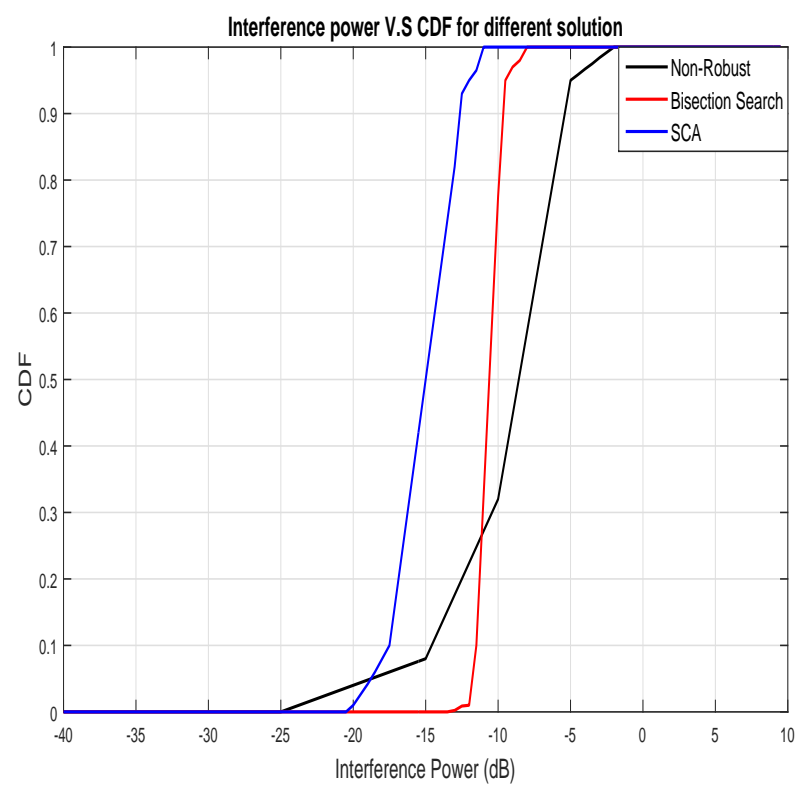

Figure 4.7: Interfernce power for different solutions versus cumulative distribution function $(\mathrm{CDF})$ at PU-R.

\subsection{Conclusion}

This chapter presents a novel procedure for the design of beamforming vectors in multicell, multigroup, multicasting CRN with non-perfect CSI for both the secondary user network and interference power network. The formulated problem for the maximization of minimum SINR at all SU-R users within specified amount of transmitted power from the SU-R base stations and limited interferences power to the PU-R is non-convex and difficult to solve as a single optimization problem. Thus, it is divided into two sub-problems, each of which is independently solved. The interference power problem is solved optimally and then integrated to the QOS problem, which can be solved either using the SCA or the bisection search method. We present simulation 
results showing that our solution methods outperform both the existing method and the non-robust solution. Further the theoretical analysis and simulation results indicate that the two methods can offer a tradeoff between low solution complexity (using the SCA method) and improved system user performance (using the bisection search method). Future work could consider a rigid method(s) for users scheduling and solve the problem using Non-orthogonal multiple access (NOMA) technique with non-perfect CSI.

\subsection{Appendices}

\subsubsection{Appendix 1: proof of feasibility and convergence of $\mathrm{P} 4$}

According to the value of $\widehat{\mathbf{R}}_{m} \in \mathbb{C}^{N \times N}$ and $\epsilon_{m}$ the resultant value of $\widehat{\mathbf{R}}_{m}-\epsilon_{m} I \in \mathbb{C}^{N \times N}$ can be Hermitian indefinite (eigenvalues positive and negative) and can be converted to PSD thus by converting every negative eigenvalues of the term $\widehat{\mathbf{R}}_{m}-\epsilon_{m} I$ to zero to satisfy the following quadratic form inequality [98] :

$$
\mathbf{x}^{H}\left(\widehat{\mathbf{R}}_{m}-\epsilon_{m} I\right)^{+} \mathbf{x} \geq 0
$$

where $x \in \mathbb{C}^{N \times 1}$ is any random vector. This is a very important condition to satisfy convergence. The following case proves the convergence and feasibility of our solution to the beamforming problem using SCA approximation method:

Case 1:The first constraint in $\mathrm{P} 5$ is 


$$
\begin{gathered}
-\left(\mathbf{w}_{k}^{(t) H}\left(\widehat{\mathbf{R}}_{m}-\epsilon_{m} \mathbf{I}_{m}\right)^{+} \mathbf{w}_{k}^{(t)}+2 \Re\left(\Delta \mathbf{W}_{k}^{H}\left(\widehat{\mathbf{R}}_{m}-\epsilon_{m} \mathbf{I}_{m}\right)^{+} \mathbf{w}_{k}^{(t)}\right)\right. \\
+\gamma^{(t)}\left(\sum_{i=1, i \neq k}^{K}\left(\mathbf{w}_{i}^{(t) H}+\Delta \mathbf{w}\right)\left(\widehat{\mathbf{R}}_{m}+\epsilon_{m} \mathbf{I}_{m}\right)\left(\mathbf{w}_{k}^{(t)}+\Delta \mathbf{w}\right)\right) \\
+\Delta \gamma\left(\sum_{i=1, i \neq k}^{K} \mathbf{w}_{k}^{(t) H}\left(\widehat{\mathbf{R}}_{m}+\epsilon_{m} \mathbf{I}_{m}\right) \mathbf{w}_{k}^{(t)}\right) \\
+\sigma_{m}^{2}\left(\Delta \gamma+\gamma^{(t)}\right) \leq 0
\end{gathered}
$$

in this case we assume that $\mathbf{w}_{i} \in \mathbb{C}^{N \times 1}$ is randomly generated matrix , $\Delta \mathbf{w}_{i}=0$ and $\Delta \gamma=0$ substituting these values in (4.32) we can obtain

$$
\frac{\mathbf{w}_{k}^{H}\left(\widehat{\mathbf{R}}_{m}-\epsilon_{m} I_{m}\right) \mathbf{w}_{k}}{\left.\sum_{i=1, i \neq k}^{K} \mathbf{w}_{i}^{H} \widehat{\mathbf{R}}_{m}\right)+\epsilon_{m} \mathbf{I}_{m} \mathbf{w}_{i}+\sigma_{m}^{2}} \geq \gamma
$$

Observe here (4.33) is the same as constraint of (4.11b) and hence when $\Delta \mathbf{w}_{i}=0$ and $\Delta \gamma=0$ then the solution is feasible

Case2 : similar to case one we assume that $\mathbf{w}_{i} \in \mathbb{C}^{N \times 1}$ is a random vector and, $\Delta \mathbf{w}_{i}=0$ but here we assume that $\Delta \gamma \neq 0$ and substitute these values in (4.32), the result will be:

$$
\frac{\mathbf{w}_{k}^{H}\left(\widehat{\mathbf{R}}_{m}-\epsilon_{m} \mathbf{I}_{m}\right)^{+} \mathbf{w}_{k}}{\sum_{i=1, i \neq k}^{K} \mathbf{w}_{i}^{H}\left(\widehat{\mathbf{R}}_{m}+\epsilon_{m} \mathbf{I}_{m}\right) \mathbf{w}_{i}+\sigma_{m}^{2}} \geq \gamma+\Delta \gamma
$$

If we assume that $\Delta \gamma<0$ then (4.33) violates P2 and by contradiction, the value of $\Delta \gamma \geq 0$ 
Case3: from cases 1 and 2 we ascertain $\Delta \gamma \geq 0$. Now consider that $\mathbf{w}_{i} \in \mathbb{C}^{N \times 1}$ and $\boldsymbol{\Delta} \mathbf{w}_{i} \in \mathbb{C}^{N \times 1}$ are any random vectors and following the procedure in [120] and substituting in (4.32) this can be reduced to:

$$
\begin{gathered}
\left(\mathbf{w}_{k}^{(t) H}\left(\widehat{\mathbf{R}}_{m}-\epsilon_{m} \mathbf{I}_{m}\right)^{+} \mathbf{w}_{k}^{(t)}+2 \Re\left(\Delta \mathbf{w}_{k}^{H}\left(\widehat{\mathbf{R}}_{m}-\epsilon_{m} \mathbf{I}_{m}\right)^{+} \mathbf{w}_{k}^{(t)}\right)\right. \\
\geq \gamma\left(\sum_{i=1, i \neq k}^{K}\left(\mathbf{w}_{k}^{(t) H}+\Delta \mathbf{w}\right)\left(\widehat{\mathbf{R}}_{m}+\epsilon_{m} \mathbf{I}_{m}\right)\left(\mathbf{w}_{k}^{(t)}+\Delta \mathbf{w}\right)+\sigma^{2}\right) \\
+\Delta \gamma\left(\sum_{i=1, i \neq k}^{K} \mathbf{w}_{k}^{(t) H}\left(\widehat{\mathbf{R}}_{m}+\epsilon_{m} \mathbf{I}_{m}\right) \mathbf{w}_{k}^{(t)}+\sigma_{m}^{2}\right) \\
\forall m \in G_{k}, \forall k \in\{1, \ldots, K\}
\end{gathered}
$$

Adding to the left hand side of (4.34) the term $\Delta \mathbf{w}_{k}^{H}\left(\widehat{\mathbf{R}}_{m}+\epsilon_{m} \mathbf{I}_{m}\right) \boldsymbol{\Delta} \mathbf{w}_{k} \geq 0$ and dividing both sides by $\sum_{i=1, i \neq k}^{K}\left(\mathbf{w}_{k}^{(t) H}+\Delta \mathbf{w}\right)\left(\widehat{\mathbf{R}}_{m}+\epsilon_{m} \mathbf{I}_{m}\right)\left(\mathbf{w}_{k}^{(t)}+\boldsymbol{\Delta} \mathbf{w}\right)+\sigma^{2}$ and define

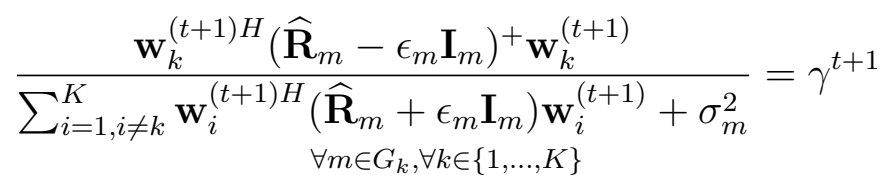

this reduces (4.34) to

$$
\begin{gathered}
\Delta \gamma \frac{\sum_{i=1, i \neq k}^{K} \mathbf{w}_{k}^{(t) H}\left(\mathbf{R}_{m}-\epsilon_{m} \mathbf{I}_{m}\right)^{+} \mathbf{w}_{k}^{(t)}+\sigma_{m}^{2}}{\sum_{i=1, i \neq k}^{K}\left(\mathbf{w}_{k}^{(t) H}+\Delta \mathbf{w}\right)\left(\widehat{\mathbf{R}}_{m}+\epsilon_{m} \mathbf{I}_{m}\right)\left(\mathbf{w}_{k}^{(t)}+\Delta \mathbf{w}\right)+\sigma^{2}} \\
+\gamma^{t} \leq \gamma^{t+1} \\
\forall m \in G_{k}, \forall k \in\{1, \ldots, K\}
\end{gathered}
$$

By the definition in (4.31), the right hand side of (4.36) is positive for any values and any new iteration satisfies $\gamma^{t+1}>\gamma^{t}$, provided that $\Delta \gamma \neq 0$. Thus, the SCA method must converge within a finite number of iterations. 


\subsubsection{APPENDIX 2: (Solution for P5 in section 4 to find $\{\Delta \mathbf{R}\}_{m=1}^{M}$}

Defining $A_{i}=\gamma \sum_{i=1, i \neq k}^{K} \mathbf{w}_{i} \mathbf{w}_{i}^{H}-\mathbf{w}_{k} \mathbf{w}_{k}^{H}$

In this proposed solution we extend Theorem 1 in [96] to solve (4.27) where the author in [96] solved a problem similar to (4.27) when $\mathbf{A}_{i}$ is positive(semi)definite. Here we extend the solution and solve the problem when the matrix $\mathbf{A}_{i}$ is indefinite. Here $\boldsymbol{\Delta} \mathbf{R}_{m}$ can be found using the following equation

$$
\Delta \mathbf{R}_{m}=\underset{\forall m \in(1, \ldots, M)}{\mathbf{U}_{p m} \boldsymbol{\Lambda}_{\Delta \mathbf{R}_{m}^{*}}} S_{m} \mathbf{U}_{p m}^{\dagger}
$$

where $\Lambda_{\Delta \mathbf{R}_{m}^{*}}$ is the diagonal eigenvalue matrix of $\Delta \mathbf{R}_{m}^{*}$ that can be determined by:

$$
\lambda_{1}\left(\Delta \mathbf{R}_{m}^{*}\right)=\epsilon_{m}\left[1+\sum_{\forall m \in(1, \ldots, M)}^{N} \frac{\left|\lambda_{n}\left(\mathbf{Y}_{m}\right)\right|}{\left|\lambda_{1}\left(\mathbf{Y}_{m}\right)\right|}\right]^{-.5}
$$

and

$$
\lambda_{n}\left(\boldsymbol{\Delta} \mathbf{R}_{m}^{*}\right)=\underset{\substack{\forall m \in(1, \ldots, M) \\\left[\lambda_{1}\left(\mathbf{Y}_{m}\right) \mid\right.}}{\left[\mid \lambda_{n}\right)} \lambda_{1}\left(\boldsymbol{\Delta} \mathbf{R}_{m}^{*}\right), n=2, \ldots . N
$$

where $\lambda_{n}\left(\boldsymbol{\Delta} \mathbf{R}_{m}^{*}\right)$ is the $n^{\text {th }}$ eigenvalue of the matrix $\boldsymbol{\Delta} \mathbf{R}_{m}^{*}$, and $\lambda_{n}\left(\mathbf{Y}_{m}\right)$ is the $n^{\text {th }}$ eigenvalue of the matrix $\mathbf{Y}_{m}$ (sorted in descending order) that can be determined by the eigen decomposition of $\mathbf{Y}\left(\mathbf{Y}_{m}=\mathbf{U}_{m} \boldsymbol{\Lambda}_{m} \mathbf{U}_{m}^{\dagger}\right)$, and $\mathbf{U}_{p m}$ can be found using 


$$
\underset{\forall m \in(1, \ldots, M)}{\mathbf{U}_{p m}=\mathbf{U}_{m} \mathbf{P}_{m}}
$$

where $\mathbf{P}_{m}$ is the permutation matrix whose location of entry is corresponding to the index of the sorted eigenvalues $\lambda_{n}\left(\mathbf{Y}_{m}\right)$. The matrix $\mathbf{Y}_{m}$ is found using:

$$
\underset{\forall m \in G_{k}}{\mathbf{Y}_{m}}=\mathbf{A}_{i}+\mathbf{Z}_{m}
$$

where $\mathbf{Z}_{m}$ in (4.42) can be found using the following optimization problem:

$$
\begin{aligned}
& \text { (P9) : } \\
& \min _{\{\mathbf{Z}\}_{m=1}^{M}} \epsilon_{m}\left\|\mathbf{A}_{i}+\mathbf{Z}_{m}\right\|_{F}+\operatorname{Tr}\left(\widehat{\mathbf{R}}_{m} \mathbf{Z}_{m}\right) \\
& \text { s.t. } \quad \mathbf{Z}_{m} \succeq 0 \\
& \forall m \in G_{k}, \forall k \in\{1, \ldots, K\}
\end{aligned}
$$

For more details regarding the proof of (4.39), (4.40), and (3.21) please see [120]. Problem P6 is convex and can be solved using the non-monotone spectral projected gradient NMSPG [121] with computational complexity of $3 N+\mathcal{O}(1)$, or a convex optimization package such as CVX [122]. 


\section{Chapter 5}

\section{Summary and Future Work}

This chapter summarizes our developed beamforming techniques for both conventional and cognitive wireless networks. It also describes some future intended works related to these networks using both convex optimization and statistical analysis methods.

\section{$5.1 \quad$ Research Summary}

Chapter 3 considered the downlink beamforming problem in the conventional wireless network when all the links between the base stations and the users are uncertain. Two solutions were developed using the SCA method. The suboptimal solution following past others' works and our proposed robust solution. The two solutions were examined through numerical simulation, and the result shows the convergence and validation of the solutions for different network parameters. Also, the result shows that the proposed solution outperforms the suboptimal one. 
Chapter four demonstrates the problem in wireless CR network and assumes the secondary links and the leakage links from the secondary transmitters to the primary receivers and the leakage links from the secondary transmitters to the primary receivers all have imperfect CSIs. Like the case in a conventional network, tow solutions were developed. For the purposed robust solution two methods were used the SCA method and the bisection search method. The results show that the bisection search method outperforms the SCA method in terms of QOS but requires higher complexity, which can offer good trade-off between performance and complexity.

\section{$5.2 \quad$ Future Work}

We are planning to extend our work towards the following directions:

\subsubsection{Channel Estimation and Channel Uncertainty}

One of the main important research subjects in a wireless network is the CSI estimation. For future work, we can develop and improve the analytical cutting plan method ACCPM that proved to be have the best performance and complexity over the known other methods. We shall use ACCPM with a different new scenario to estimate the CSI with better complexity and higher performance.

Although more advance methods have been used to estimate CSI, the CSI still suffers from the uncertainty due to the nature of the wireless channel. In future work, we will consider the uncertainty in two different ways. The first is by using stochastic molding of the system instead of deterministic modeling this occurs when only the 
statistical information of the CSI is available for system analysis. The second way is to consider a more complicated system model and a more sensitive objective function, such as a massive MIMO system and outage probability objective function.

\subsubsection{Non Orthogonal Multiple Access(NOMA)}

NOMA is a new beamforming technique that is used in $5 \mathrm{G}$ wireless communication system that applies massive MIMO and mmwave. In this technique, more than one user can share the same beamforming signal using a special technique. In subsequent work, both beamforming models ( in perfect CSI and non-perfect CSI) will be considered and studied deeply.

\subsubsection{Cognitive Network and Model Order Selection}

The CR network considered in this work is an underlay type that is not considered whether the PU is on or off. The second type pf CR network is an overlay which is only communicated when the PU is off. For the overlay CR network, a fusion center is applying at the secondary network to determine the of PU or PUs and release the frequency to the $\mathrm{CR}$ network(s). this fusion center must apply an algorithm known as a model order selection to determine the off PU(s). in future work, we shall consider the study and design a model selection order to examine more than one PU and specify the inactive PUs. Our algorithm will apply Bayesian approaches that approve better performance and less complexity than conventional statistical methods. 


\section{Bibliography}

[1] M. Longair, ... a paper... i hold to be great guns': a commentary on maxwell (1865)'a dynamical theory of the electromagnetic field, Philosophical Transactions of the Royal Society A: Mathematical, Physical and Engineering Sciences 373 (2039) (2015) 20140473.

[2] K. Sengar, N. Rani, A. Singhal, D. Sharma, S. Verma, T. Singh, Study and capacity evaluation of siso, miso and mimo rf wireless communication systems, arXiv preprint arXiv:1403.7774.

[3] S. Srivastava, M. Singh, S. Gupta, Wireless sensor network: a survey, in: 2018 International Conference on Automation and Computational Engineering (ICACE), IEEE, 2018, pp. 159-163.

[4] M. K. Singh, S. I. Amin, S. A. Imam, V. K. Sachan, A. Choudhary, A survey of wireless sensor network and its types, in: 2018 International Conference on Advances in Computing, Communication Control and Networking (ICACCCN), IEEE, 2018, pp. 326-330.

[5] I. F. Akyildiz, W. Su, Y. Sankarasubramaniam, E. Cayirci, A survey on sensor networks, IEEE Communications magazine 40 (8) (2002) 102-114. 
[6] D. Kumar, K. Ramalakshmi, Survey on cognitive wireless sensor network, in: 2018 Second International Conference on Electronics, Communication and Aerospace Technology (ICECA), IEEE, 2018, pp. 1885-1889.

[7] M. F. Othman, K. Shazali, Wireless sensor network applications: A study in environment monitoring system, Procedia Engineering 41 (2012) 1204-1210.

[8] V. K. Sachan, S. A. Imam, M. Beg, Energy-efficient communication methods in wireless sensor networks: A critical review, International Journal of Computer Applications 39 (17) (2012) 35-48.

[9] J. L. Hill, System architecture for wireless sensor networks, Ph.D. thesis, University of California, Berkeley (2003).

[10] A. Nechibvute, A. Chawanda, P. Luhanga, Piezoelectric energy harvesting devices: an alternative energy source for wireless sensors, Smart Materials Research 2012.

[11] V. Raghunathan, C. L. Pereira, M. B. Srivastava, R. K. Gupta, Energy-aware wireless systems with adaptive power-fidelity tradeoffs, IEEE Transactions on Very Large Scale Integration (VLSI) Systems 13 (2) (2005) 211-225.

[12] P. Jin, X. Hao, X. Wang, L. Yue, Energy-efficient task scheduling for cpuintensive streaming jobs on hadoop, IEEE Transactions on Parallel and Distributed Systems 30 (6) (2018) 1298-1311.

[13] V. Raghunathan, C. Schurgers, S. Park, M. B. Srivastava, Energy-aware wireless microsensor networks, IEEE Signal processing magazine 19 (2) (2002) 40-50. 
[14] F. L. Lewis, Wireless sensor networks, Smart environments: technologies, protocols, and applications (2004) 11-46.

[15] D.-S. Lee, Y.-H. Liu, C.-R. Lin, A wireless sensor enabled by wireless power, Sensors 12 (12) (2012) 16116-16143.

[16] J. M. Gilbert, F. Balouchi, Comparison of energy harvesting systems for wireless sensor networks, International Journal of automation and computing 5 (4) (2008) 334-347.

[17] T. Kamsuvan, Power management in wireless sensor networks (wsns), Ph.D. thesis, Brunel University London (2016).

[18] Y. Shi, L. Xie, Y. T. Hou, H. D. Sherali, On renewable sensor networks with wireless energy transfer, in: 2011 Proceedings IEEE INFOCOM, IEEE, 2011, pp. 1350-1358.

[19] L. Xie, Y. Shi, Y. T. Hou, A. Lou, Wireless power transfer and applications to sensor networks, IEEE Wireless Communications 20 (4) (2013) 140-145.

[20] A. K. Sah, D. R. Pant, Analysis and optimization of wireless power transfer link, in: Proceedings of IOE Graduate Conference, 2014, p. 83.

[21] R. Du, A. Ozcelikkale, C. Fischione, M. Xiao, Optimal energy beamforming and data routing for immortal wireless sensor networks, in: 2017 IEEE International Conference on Communications (ICC), IEEE, 2017, pp. 1-6.

[22] J. B. Andersen, T. S. Rappaport, S. Yoshida, Propagation measurements and models for wireless communications channels, IEEE Communications Magazine 33 (1) (1995) 42-49. 
[23] M. F. Iskander, Z. Yun, Propagation prediction models for wireless communication systems, IEEE Transactions on microwave theory and techniques 50 (3) (2002) 662-673.

[24] J. S. Seybold, Introduction to RF propagation, John Wiley \& Sons, 2005.

[25] W. Debus, L. Axonn, Rf path loss \& transmission distance calculations, Axonn, LLC.

[26] V. Abhayawardhana, I. Wassell, D. Crosby, M. Sellars, M. Brown, Comparison of empirical propagation path loss models for fixed wireless access systems, in: 2005 IEEE 61st Vehicular Technology Conference, Vol. 1, IEEE, 2005, pp. 73-77.

[27] M. Alim, M. Rahman, M. Hossain, A. A. Nahid, Analysis of large scale propagation models for mobile communications in urban area, arXiv preprint arXiv:1002.2187.

[28] F. M. Landstorfer, Wave propagation models for the planning of mobile communication networks, in: 1999 29th European Microwave Conference, Vol. 1, IEEE, 1999, pp. 1-6.

[29] E. Biglieri, R. Calderbank, A. Constantinides, A. Goldsmith, A. Paulraj, H. V. Poor, MIMO wireless communications, Cambridge university press, 2007.

[30] W. Hardjawana, R. Li, B. Vucetic, Y. Li, X. Yang, A new iterative channel estimation for high mobility mimo-ofdm systems, in: 2010 IEEE 71st Vehicular Technology Conference, IEEE, 2010, pp. 1-5. 
[31] N. Aboutorab, W. Hardjawana, B. Vucetic, A new iterative doppler-assisted channel estimation joint with parallel ici cancellation for high-mobility mimo-ofdm systems, IEEE Transactions on Vehicular Technology 61 (4) (2012) 1577-1589.

[32] N. Wang, G. Gui, Z. Zhang, T. Tang, J. Jiang, A novel sparse channel estimation method for multipath mimo-ofdm systems, in: 2011 IEEE Vehicular Technology Conference (VTC Fall), IEEE, 2011, pp. 1-5.

[33] Y. Liu, S. Sezginer, Iterative compensated mmse channel estimation in lte systems, in: 2012 IEEE International Conference on Communications (ICC), IEEE, 2012, pp. 4862-4866.

[34] J. Ketonen, M. Juntti, J. Ylioinas, J. R. Cavallaro, Decision-directed channel estimation implementation for spectral efficiency improvement in mobile mimoofdm, Journal of Signal Processing Systems 79 (3) (2015) 233-245.

[35] R. Zhang, On active learning and supervised transmission of spectrum sharing based cognitive radios by exploiting hidden primary radio feedback, IEEE Transactions on Communications 58 (10) (2010) 2960-2970.

[36] Y. Noam, A. Manolakos, A. J. Goldsmith, Null space learning with interference feedback for spatial division multiple access, IEEE Transactions on Wireless Communications 13 (10) (2014) 5699-5715.

[37] K.-Y. Wang, N. Jacklin, Z. Ding, C.-Y. Chi, Robust \{MISO Transmit\} optimization under outage-based qos constraints in two-tier heterogeneous networks, IEEE transactions on wireless communications 12 (4) (2013) 1883-1897. 
[38] S. Ma, D. Sun, Chance constrained robust beamforming in cognitive radio networks, IEEE Communications Letters 17 (1) (2012) 67-70.

[39] X. Kang, R. Zhang, Y.-C. Liang, H. K. Garg, Optimal power allocation strategies for fading cognitive radio channels with primary user outage constraint, IEEE Journal on Selected Areas in Communications 29 (2) (2011) 374-383.

[40] D. P. Palomar, J. M. Cioffi, M. A. Lagunas, Uniform power allocation in mimo channels: A game-theoretic approach, IEEE Transactions on Information Theory 49 (7) (2003) 1707-1727.

[41] H. Yi, Nullspace-based secondary joint transceiver scheme for cognitive radio mimo networks using second-order statistics, in: 2010 IEEE International Conference on Communications, IEEE, 2010, pp. 1-5.

[42] F. Gao, R. Zhang, Y.-C. Liang, X. Wang, Design of learning-based mimo cognitive radio systems, IEEE Transactions on Vehicular Technology 59 (4) (2010) $1707-1720$.

[43] A. Manolakos, Y. Noam, A. J. Goldsmith, Interference due to null space mismatch in cooperative multipoint mimo cellular networks, in: 2014 IEEE International Conference on Communications (ICC), IEEE, 2014, pp. 5148-5153.

[44] K.-L. A. Yau, P. Komisarczuk, P. D. Teal, A context-aware and intelligent dynamic channel selection scheme for cognitive radio networks, in: 2009 4th International Conference on Cognitive Radio Oriented Wireless Networks and Communications, IEEE, 2009, pp. 1-6. 
[45] I. . W. Group, et al., Ieee standard for information technology-local and metropolitan area networks - specific requirements - part 11: Wireless lan medium access control (mac) and physical layer (phy) specifications amendment 6: Wireless access in vehicular environments; ieee $802.11 \mathrm{p}$, IEEE Standards Association: Piscataway, NJ, USA.

[46] Y. Tawk, M. Bkassiny, G. El-Howayek, S. Jayaweera, K. Avery, C. Christodoulou, Reconfigurable front-end antennas for cognitive radio applications, IET microwaves, antennas \& propagation 5 (8) (2011) 985-992.

[47] S. K. Jayaweera, C. Mosquera, A dynamic spectrum leasing (dsl) framework for spectrum sharing in cognitive radio networks, in: 2009 Conference Record of the Forty-Third Asilomar Conference on Signals, Systems and Computers, IEEE, 2009, pp. 1819-1823.

[48] A. B. Gershman, N. D. Sidiropoulos, S. Shahbazpanahi, M. Bengtsson, B. Ottersten, Convex optimization-based beamforming, IEEE Signal Processing Magazine 27 (3) (2010) 62-75.

[49] Y. Rong, S. A. Vorobyov, A. B. Gershman, A robust linear receiver for uplink multi-user mimo systems based on probability-constrained optimization and second-order cone programming, in: Processing Workshop Proceedings, 2004 Sensor Array and Multichannel Signal, IEEE, 2004, pp. 153-157.

[50] B. Friedlander, On transmit beamforming for mimo radar, IEEE Transactions on Aerospace and Electronic Systems 48 (4) (2012) 3376-3388. 
[51] C. Zhang, Y. Huang, Y. Jing, S. Jin, L. Yang, Sum-rate analysis for massive mimo downlink with joint statistical beamforming and user scheduling, IEEE Transactions on Wireless Communications 16 (4) (2017) 2181-2194.

[52] F. Liu, J. Wang, R. Du, L. Peng, P. Chen, A second-order cone programming approach for robust downlink beamforming with power control in cognitive radio networks, Progress in Electromagnetics Research 18 (2011) 221-231.

[53] K. L. Law, C. Masouros, M. Pesavento, Transmit beamforming for interference exploitation in the underlay cognitive radio z-channel, arXiv preprint arXiv:1606.06504.

[54] J.-B. Hiriart-Urruty, C. Lemaréchal, Fundamentals of convex analysis, Springer Science \& Business Media, 2012.

[55] R. T. Rockafellar, Convex analysis, no. 28, Princeton university press, 1970.

[56] M. S. Bazaraa, H. D. Sherali, C. M. Shetty, Nonlinear programming: theory and algorithms, John Wiley \& Sons, 2013.

[57] D. G. Luenberger, Y. Ye, et al., Linear and nonlinear programming, Vol. 2, Springer, 1984.

[58] J. W. Demmel, Applied numerical linear algebra, Vol. 56, Siam, 1997.

[59] H. Hindi, A tutorial on convex optimization, in: Proceedings of the 2004 American Control Conference, Vol. 4, IEEE, 2004, pp. 3252-3265.

[60] S. Bubeck, et al., Convex optimization: Algorithms and complexity, Foundations and Trends@ in Machine Learning 8 (3-4) (2015) 231-357. 
[61] J. Borwein, A. S. Lewis, Convex analysis and nonlinear optimization: theory and examples, Springer Science \& Business Media, 2010.

[62] G. Scutari, Y. Sun, Parallel and distributed successive convex approximation methods for big-data optimization, in: Multi-agent Optimization, Springer, 2018, pp. $141-308$.

[63] M. Razaviyayn, Successive convex approximation: Analysis and applications.

[64] A. Lin, et al., Binary search algorithm, WikiJournal of Science 2 (1) (2019) 1.

[65] R. M. Freund, The steepest descent algorithm for unconstrained optimization and a bisection line-search method, Journal of Massachusetts Institute of Technology. United States of america.

[66] A. Goldsmith, Wireless communications. Cambridge university press, 2005.

[67] N. D. Sidiropoulos, T. N. Davidson, and Z.-Q. Luo, "Transmit beamforming for physical-layer multicasting," IEEE Trans. Signal Process., vol. 54, no. 6, pp. 2239-2251, 2006.

[68] S. X. Wu, W.-K. Ma, and A. M.-C. So, "Physical-layer multicasting by stochastic transmit beamforming and alamouti space-time coding," IEEE Trans. Signal Process., vol. 61, no. 17, pp. 4230-4245, 2013.

[69] D. Christopoulos, S. Chatzinotas, and B. Ottersten, "Weighted fair multicast multigroup beamforming under per-antenna power constraints," IEEE Trans. Signal Process., vol. 62, no. 19, pp. 5132-5142, 2014. 
[70] N. Bornhorst and M. Pesavento, "An iterative convex approximation approach for transmit beamforming in multi-group multicasting," in Signal Processing Advances in Wireless Communications (SPAWC), 2011 IEEE 12th Int. l Workshop on. IEEE, 2011, pp. 426-430.

[71] K. L. Law, X. Wen, M. T. Vu, and M. Pesavento, "General rank multiuser downlink beamforming with shaping constraints using real-valued ostbc," IEEE Trans. Signal Process., vol. 63, no. 21, pp. 5758-5771, 2015.

[72] B. Gopalakrishnan and N. D. Sidiropoulos, "Cognitive transmit beamforming from binary csit," IEEE Trans. Wireless Commun., vol. 14, no. 2, pp. 895-906, 2015.

[73] Y. Noam and A. J. Goldsmith, "The one-bit null space learning algorithm and its convergence," IEEE Trans. Signal Process., vol. 61, no. 24, pp. 6135-6149, 2013.

[74] S. Loyka and C. D. Charalambous, "Novel matrix singular value inequalities and their applications to uncertain mimo channels," IEEE Transactions on Inf. Theory, vol. 61, no. 12, pp. 6623-6634, 2015.

[75] A. Patel, S. Biswas, and A. K. Jagannatham, "Optimal glrt-based robust spectrum sensing for mimo cognitive radio networks with csi uncertainty." IEEE Trans. Signal Process., vol. 64, no. 6, pp. 1621-1633, 2016.

[76] B. Liu, Y. Cheng, and Q. Zhou, "Robust rank-two beamforming for multicell multigroup multicast," IET Commun., vol. 10, no. 3, pp. 283-291, 2016. 
[77] W. Xu, Y. Cui, H. Zhang, G. Y. Li, and X. You, "Robust beamforming with partial channel state information for energy efficient networks," IEEE Journal on Selected Areas in Commun., vol. 33, no. 12, pp. 2920-2935, 2015.

[78] K. Cumanan, R. Krishna, V. Sharma, and S. Lambotharan, "Robust interference control techniques for multiuser cognitive radios using worst-case performance optimization," in Signals, Systems and Computers, 2008 42nd Asilomar Conference on. IEEE, 2008, pp. 378-382.

[79] S. Parsaeefard and A. R. Sharafat, "Robust worst-case interference control in underlay cognitive radio networks," IEEE Trans. Veh. Technol., vol. 61, no. 8, pp. 3731-3745, 2012.

[80] J. Wang and D. P. Palomar, "Worst-case robust mimo transmission with imperfect channel knowledge," IEEE Trans. Signal Process., vol. 57, no. 8, pp. 30863100, 2009.

[81] J. Wang, M. Bengtsson, B. Ottersten, and D. P. Palomar, "Robust mimo precoding for several classes of channel uncertainty," IEEE Trans. Signal Process., vol. 61, no. 12, pp. 3056-3070, 2013.

[82] M. H. Al-Ali and K. Ho, "Transmit precoding in underlay mimo cognitive radio with unavailable or imperfect knowledge of primary interference channel," IEEE Trans. Wireless Commun., vol. 15, no. 8, pp. 5143-5155, 2016.

[83] M. Biguesh, S. Shahbazpanahi, and A. B. Gershman, "Robust downlink power control in wireless cellular systems," EURASIP J. l on Wireless Commun., vol. 2004, no. 2, pp. 261-272, 2004. 
[84] E. Karipidis, N. D. Sidiropoulos, and Z.-Q. Luo, "Quality of service and max-min fair transmit beamforming to multiple cochannel multicast groups," IEEE Trans. Signal Process., vol. 56, no. 3, pp. 1268-1279, 2008.

[85] A. Schad and M. Pesavento, "Max-min fair transmit beamforming for multigroup multicasting," in Smart Antennas (WSA), 2012 Int. l ITG Workshop on. IEEE, 2012, pp. 115-118.

[86] M. Bengtsson and B. Ottersten, "Optimal downlink beamformingusing semidefinite optimization," in 37th Ann. Allerton Conf. on Commu. , Control, and Computing, 1999, pp. 987-996.

[87] M. Bengtsson, B. Ottersten, Optimal and suboptimal transmit beamforming, 2001 .

[88] M. F. Hanif, P. J. Smith, M.-S. Alouini, Sinr balancing in the downlink of cognitive radio networks with imperfect channel knowledge, in: 2010 Proceedings of the Fifth International Conference on Cognitive Radio Oriented Wireless Networks and Communications, IEEE, 2010, pp. 1-5.

[89] U. L. Wijewardhana, M. Codreanu, M. Latva-aho, and A. Ephremides, "A robust beamformer design for underlay cognitive radio networks using worst case optimization," EURASIP Journal on Wireless Commun., vol. 2014, no. 1, p. 37, 2014.

[90] H. Xu, B. Zhu, J. Liu, and A. Zhou, "Robust beamforming design for secure multiuser miso interference channel," IEEE Commun. Lett., vol. 21, no. 4, pp. 833-836, 2017. 
[91] I. Wajid, Y. C. Eldar, and A. Gershman, "Robust downlink beamforming using covariance channel state information," in Acoust., Speech and Signal Process., 2009. ICASSP 2009. IEEE Int. Conf. on. IEEE, 2009, pp. 2285-2288.

[92] E. A. Gharavol, Y.-C. Liang, and K. Mouthaan, "Robust downlink beamforming in multiuser miso cognitive radio networks with imperfect channel-state information," IEEE Trans. Veh. Technol., vol. 59, no. 6, pp. 2852-2860, 2010.

[93] K. L. Law, I. Wajid, and M. Pesavento, "Robust downlink beamforming in multigroup multicasting using trace bounds on the covariance mismatches," in Acoust. , Speech and Signal Process. (ICASSP), 2012 IEEE Int. Conf. on. IEEE, 2012, pp. 3229-3232.

[94] I. Wajid, M. Pesavento, Y. C. Eldar, D. Ciochina, Robust downlink beamforming with partial channel state information for conventional and cognitive radio networks, IEEE Transactions on signal processing 61 (14) (2013) 3656-3670.

[95] G. Zheng, K.-K. Wong, B. Ottersten, Robust cognitive beamforming with bounded channel uncertainties, IEEE Transactions on Signal Processing 57 (12) (2009) 4871-4881.

[96] M. H. Al-Ali, D. K. Ho, Precoding for mimo channels in cognitive radio networks with csi uncertainties and for mimo compound capacity, IEEE Transactions on Signal Processing 65 (15) (2017) 3976-3989.

[97] O. Mehanna, K. Huang, B. Gopalakrishnan, A. Konar, and N. D. Sidiropoulos, "Feasible point pursuit and successive approximation of non-convex qcqps," IEEE Signal Process. Lett., vol. 22, no. 7, pp. 804-808, 2015. 
[98] D. S. Bernstein, Matrix mathematics: theory, facts, and formulas, Princeton university press, 2009.

[99] E. G. Birgin ,J. M Martínez and M Raydan, "Spectral projected gradient methods: review and perspectives," J. Stat. Softw, vol. 60, no. 3, pp. 1-21, 2014.

[100] V. Valenta, Z. Fedra, R. Marsalek, G. Baudoin, M. Villegas, Towards cognitive radio networks: Spectrum utilization measurements in suburb environment, in: 2009 IEEE Radio and Wireless Symposium, IEEE, 2009, pp. 352-355.

[101] Z. M. Bakhsh, J. zeraatkar Moghadam, M. Ardebilipour, Cognitive radio and cooperation among the primary and secondary users, in: 2019 27th Iranian Conference on Electrical Engineering (ICEE), IEEE, 2019, pp. 1601-1605.

[102] S. Ivanenko, V. Bezruk, Methods of detecting of unknown signals in cognitive radio networks, in: 2016 Third International Scientific-Practical Conference Problems of Infocommunications Science and Technology (PIC S\&T), IEEE, 2016, pp. 199-200.

[103] S. Force, Spectrum policy task force report, Federal Communications Commission ET Docket 02, vol. 135.

[104] J. Mitola, G. Q. Maguire, et al., Cognitive radio: making software radios more personal, IEEE personal communications 6 (4) (1999) 13-18.

[105] F. Xu, L. Zhang, Z. Zhou, Y. Ye, Architecture for next-generation reconfigurable wireless networks using cognitive radio, in: 2008 3rd International Conference on Cognitive Radio Oriented Wireless Networks and Communications (CrownCom 2008), IEEE, 2008, pp. 1-5. 
[106] B. A. Fette, Cognitive radio technology, Academic Press, 2009.

[107] E. Biglieri, A. J. Goldsmith, L. J. Greenstein, N. B. Mandayam, H. V. Poor, Principles of cognitive radio, Cambridge University Press, 2013.

[108] M. Aishwarya, S. Kirthiga, Relay assisted cooperative communication for wireless sensor networks, in: 2018 Second International Conference on Advances in Electronics, Computers and Communications (ICAECC), IEEE, 2018, pp. 1-6.

[109] R. Tandra, A. Sahai, Fundamental limits on detection in low snr under noise uncertainty, in: 2005 International Conference on Wireless Networks, Communications and Mobile Computing, Vol. 1, IEEE, 2005, pp. 464-469.

[110] D. Cabric, A. Tkachenko, R. W. Brodersen, Spectrum sensing measurements of pilot, energy, and collaborative detection, in: MILCOM 2006-2006 IEEE Military Communications conference, IEEE, 2006, pp. 1-7.

[111] S. M. Kay, Fundamentals of statistical signal processing, Prentice Hall PTR, 1993.

[112] W. A. Gardner, Exploitation of spectral redundancy in cyclostationary signals, IEEE Signal processing magazine 8 (2) (1991) 14-36.

[113] M. Al-Amidie, A. Al-Asadi, A. C. Micheas, N. E. Islam, Spectrum sensing based on bayesian generalised likelihood ratio for cognitive radio systems with multiple antennas, IET Communications 13 (3) (2018) 305-311.

[114] H. Du, T. Ratnarajah, Robust joint signal and interference alignment for mimo cognitive radio network, in: 2012 IEEE Wireless Communications and Networking Conference (WCNC), IEEE, 2012, pp. 448-452. 
[115] B. Xie, Y. Li, H. Minn, A. Nosratinia, Adaptive interference alignment with csi uncertainty, IEEE Transactions on Communications 61 (2) (2013) 792-801.

[116] F. Gao, R. Zhang, Y.-C. Liang, X. Wang, Multi-antenna cognitive radio systems: Environmental learning and channel training, in: 2009 IEEE International Conference on Acoustics, Speech and Signal Processing, IEEE, 2009, pp. 2329 2332 .

[117] B. Huang, G. Zhao, L. Li, X. Zhou, Z. Chen, Non-cooperative cross-channel gain estimation using full-duplex amplify-and-forward relaying in cognitive radio networks, in: 2016 IEEE International Conference on Acoustics, Speech and Signal Processing (ICASSP), IEEE, 2016, pp. 3636-3640.

[118] A. Tsakmalis, S. Chatzinotas, B. Ottersten, Constrained bayesian active learning of interference channels in cognitive radio networks, IEEE Journal of Selected Topics in Signal Processing 12 (1) (2017) 6-19.

[119] K. Cumanan, L. Musavian, S. Lambotharan, A. B. Gershman, Sinr balancing technique for downlink beamforming in cognitive radio networks, IEEE Signal Processing Letters 17 (2) (2009) 133-136.

[120] A. Al-Asadi, M. Al-Amidie, A. C. Micheas, R. G. McGarvey, N. E. Islam, Worst case fair beamforming for multiple multicast groups in multicell networks, IET Communications 13 (6) (2018) 664-671.

[121] E. G. Birgin, J. M. Martínez, M. Raydan, Nonmonotone spectral projected gradient methods on convex sets, SIAM Journal on Optimization 10 (4) (2000) 1196-1211. 
[122] S. Boyd, L. Vandenberghe, Convex optimization, Cambridge university press, 2004. 


\section{VITA}

Ahmed Abid-Awn Al-Asadi was born on February 141969 in Baghdad, Iraq. He received the B.Sc. and M.Sc. degrees in Electrical Engineering from University of Technology, Baghdad, Iraq, in 1993 and 1996, respectively. He joined the Electrical and Computer Engineering department at the University of Missouri-Columbia on January 2014 and since then he has begun working with Prof. Nas Islam on his research. He received master of engineering degree in electrical engineering in DEC 2020 and He expects to receive the Doctor of Philosophy degree on May 2021.

Al-Asadi is married to Mrs Zinab Naser Al-Lami with four kids: Ali, Zahra, Muhammad, and Serraa. After completing the PhD requirements, he will return back to his country and serve as a lecturer in the Information technology center, University Technology-Baghdad, Iraq. 